\title{
Infinitesimal Automorphisms of VB-Groupoids and Algebroids
}

\author{
Chiara Esposito*, \\ Dipartimento di Matematica \\ Università degli Studi di Salerno \\ via Giovanni Paolo II, 123 \\ 84084 Fisciano (SA) \\ Italy \\ Alfonso Giuseppe Tortorella \\ Department of Mathematics \\ Katholieke Universiteit (KU) Leuven \\ Celestijnenlaan 200B box 2400 \\ 3001 Leuven \\ Belgium \\ Luca Vitagliano \\ Dipartimento di Matematica \\ Università degli Studi di Salerno \\ via Giovanni Paolo II, 123 \\ 84084 Fisciano (SA) \\ Italy
}

\begin{abstract}
VB-groupoids and algebroids are vector bundle objects in the categories of Lie groupoids and Lie algebroids respectively, and they are related via the Lie functor. VB-groupoids and algebroids play a prominent role in Poisson and related geometries. Additionally, they can be seen as models for vector bundles over singular spaces. In this paper we study their infinitesimal automorphisms, i.e. vector fields on them generating a flow by diffeomorphisms preserving both the linear and the groupoid/algebroid structures. For a special class of VB-groupoids/algebroids coming from representations of Lie groupoids/algebroids, we prove that infinitesimal automorphisms are the same as multiplicative sections of a certain derivation VB-groupoid/algebroid.
\end{abstract}

\footnotetext{
* chesposito@unisa.it

$\dagger$ alfonsogiuseppe.tortorella@kuleuven. be

${ }^{\ddagger}$ Ivitagliano@unisa.it
} 


\section{Contents}

1 Preliminaries 5

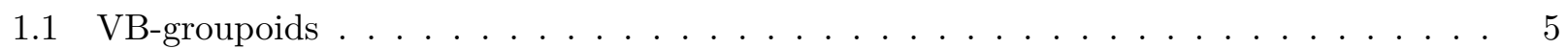

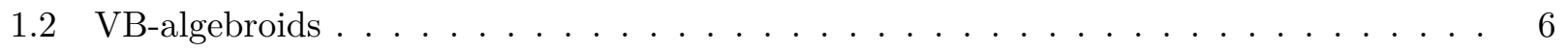

1.3 Differentiation and integration $\ldots \ldots \ldots \ldots \ldots \ldots$

\begin{tabular}{|lll}
2 & Infinitesimal automorphisms of VB-groupoids and algebroids & 10
\end{tabular}

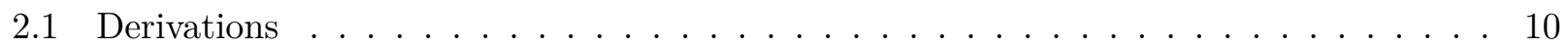

2.2 Infinitesimal automorphisms and multiplicative derivations $\ldots \ldots \ldots \ldots$

2.3 The linear deformation complex of a VB-groupoid/algebroid . . . . . . . . . . . . 20

\begin{tabular}{|lll}
3 & Multiplicative derivations of trivial-core VB-groupoids and algebroids & $\mathbf{2 7}$
\end{tabular}

3.1 VB-groupoids/algebroids and representations $\ldots \ldots \ldots \ldots \ldots \ldots$

3.2 The gauge algebroid of trivial-core/full-core VB-groupoids/algebroids $\ldots \ldots \ldots$

3.3 Multiplicative derivations as multiplicative sections $\ldots \ldots \ldots \ldots$. . . . . . . . 34

\begin{tabular}{|lll|}
\hline & A 2-term representation up to homotopy from a plain representation & 37
\end{tabular}

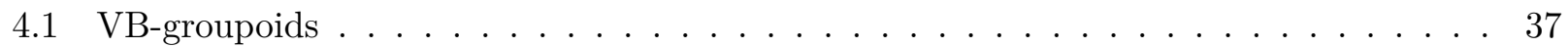

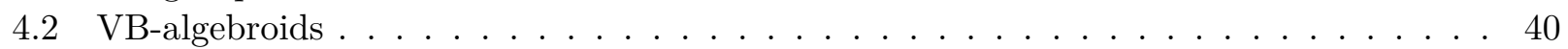

\begin{tabular}{ll}
\hline References & 42
\end{tabular} 


\section{Introduction}

This is the first in a series of papers aiming at studying (multi)differential operators (DO) and jets on Lie groupoids. Here we study a class of first order DOs, namely derivations of vector bundles (also known as covariant differential operators). Derivations can be conveniently regarded as infinitesimal automorphisms of the vector bundle structure. Thus, in the realm of Lie groupoids, it is natural to look at infinitesimal automorphisms of VB-groupoids and their infinitesimal counterparts, VBalgebroids.

VB-groupoids are groupoid objects in the category of vector bundles or, equivalently, vector bundle objects in the category of Lie groupoids [7, 28, 46. They play an important role in Poisson geometry and, more generally, in Lie groupoid theory. The tangent and the cotangent bundle of a Lie groupoid provide canonical examples. More generally, VB-groupoids can be seen as intrinsic models of twoterm representations up to homotopy of Lie groupoids [28. For instance, the whole information about the adjoint representation (up to homotopy) of a Lie groupoid $[2]$ is encoded by its tangent VB-groupoid. Accordingly, deformations of a Lie groupoid $G$ are controlled by the VB-groupoid complex of $T G$ [14.

The infinitesimal counterparts of VB-groupoids are VB-algebroids, i.e. Lie algebroid objects in the category of vector bundles or, equivalently, vector bundle objects in the category of Lie algebroids [7, 23, 27, 46]. The tangent and the cotangent bundle of a Lie algebroid provide canonical examples. Similarly to VB-groupoids, VB-algebroids can be seen as intrinsic models of two-term representations up to homotopy of Lie algebroids [27. The adjoint representation (up to homotopy) of a Lie algebroid is encoded by its tangent VB-algebroid and deformations of a Lie algebroid $A$ are controlled by the $V B$-algebroid complex of $T A[15]$. Additionally, the deformation complex of a Lie groupoid $G$ and the deformation complex of its Lie algebroid $A$ are intertwined by a Van Est map [12,64] (see also [5, 9] for homogeneous versions of the Van Est theorem), which is yet another manifestation of the action of the Lie functor [14].

Because of their importance in Lie groupoid/algebroid theory, it is natural to look at infinitesimal automorphisms of VB-groupoids and VB-algebroids and at how the Lie functor intertwines them. Notice that infinitesimal automorphisms of a Lie groupoid are the same as multiplicative vector fields on it 47. Similarly, infinitesimal automorphisms of a VB-groupoid are (equivalent to) its multiplicative derivations. Multiplicative vector fields on Lie groupoids are important objects. In particular, their theory includes the classical liftings of a vector field on the tangent and the cotangent bundle, which are of general interest in differential geometry [47. We expect multiplicative derivations to have similar importance. In this respect, this paper is also part of the research line aiming at studying multiplicative structures on Lie groupoids and their infinitesimal counterparts (see 38 for a recent review), i.e. those geometric data which are compatible with the groupoid, resp. algebroid, structure.

The study of multiplicative structures was originally motivated by Poisson-Lie groups [21] and symplectic groupoids $11,63,67,68$ and was pursued by several authors. Recent research has concentrated on multiplicative structures of tensorial type: multiplicative differential forms [6] and multivectors [29], multiplicative $(1,1)$ tensors [56], multiplicative generalized complex structures [36 (see also [3,13]), and, encompassing all the previous ones, generic multiplicative tensors [8, with few exceptions: multiplicative foliations [33,35], multiplicative Dirac structures [34,52,53], multiplicative vector-bundle valued differential forms [16,22. Non-tensorial multiplicative structures (other than the few examples listed above) have not been considered yet. In particular, a systematic investigation (including a definition) of multiplicative (multi)DOs, multiplicative jets, and their infinitesimal counterparts, is still unavailable.

There are two additional motivations to study multiplicative (multi)DOs and jets. One comes from Jacobi geometry and is represented, on one hand, by Jacobi-Lie groups [31], a wide generalization of Poisson-Lie groups and, on another hand, contact groupoids (i.e. Lie groupoids equipped with a multiplicative contact structure $[17,37,43]$ ). A Jacobi structure on a manifold $M$ is a line bundle $L \rightarrow$ 
$M$ together with a Lie bracket on its sections which is also a first order biDO (see, e.g., [48]). Jacobi structures encompass Poisson, contact, and locally conformal symplectic structures as particular cases. The case when the line bundle $L \rightarrow M$ is equipped with a global trivialization has often been an object of study. In particular Iglesias and Marrero adopt this approach to their definition of a Jacobi groupoid, i.e. a Lie groupoid with a compatible Jacobi structure [30,32. However, probably, this is not the best choice from a conceptual point of view (see, e.g., [4]). Not to mention that there are important Jacobi structures whose underlying line bundle is not at all trivial. In [4 the authors study Jacobi manifolds intrinsically, viewing them as homogeneous Poisson manifolds, in particular they define Jacobi groupoids as homogeneous Poisson groupoids. There is an alternative, more algebraic approach, requiring the technology of derivations of vector bundles (see, e.g. [41, 58]). In particular, in order to apply the latter approach to a Jacobi groupoid, one needs to understand multiplicative (multi)derivations of VB-groupoids first. Here, we begin this program by studying multiplicative derivations of a VB-groupoid. Multiplicative multiderivations will be addressed in a subsequent paper [24] (see also [61, Section 2.7]).

Our second motivation comes from the geometric theory of PDEs. Geometrically, a PDE is a submanifold in a jet space. Symmetries provide important geometric invariants of a PDE. Symmetries of a PDE form a Lie pseudogroup and the latter can be seen as a Lie subgroupoid of a jet groupoid. There are several natural (multi)DOs and jets attached to a jet space, in particular a jet groupoid 10, 16, 54, 66. One should expect that natural DOs/jets on jet groupoids are also multiplicative, so falling into the class our program aims to define and study. Additionally, zero loci of multiplicative DOs will provide an important class of PDEs on sections of vector bundles over Lie groupoids.

Finally, it is worth to mention that it has been recently shown that VB-groupoids can be seen as linear atlases on vector bundles over differentiable stacks [18, so paving the way for a theory of vector bundles over singular spaces such as orbifolds, leaf spaces of foliations, orbit spaces of group actions, etc. This paper can then be seen as a study of derivations of vector bundles over stacks.

The paper is divided into four main sections. Section 1 is a short review of VB-groupoids and VBalgebroids. Besides the book by Mackenzie [46], our main references for this material are $7,27,28$. In Section 2 we define and begin our study of multiplicative derivations. Let $\mathcal{W} \rightrightarrows E$ be a VB-groupoid over a Lie groupoid $G \rightrightarrows M$, and let $W \Rightarrow E$ be a VB-algebroid over a Lie algebroid $A \Rightarrow M$. We also write $(\mathcal{W} \rightrightarrows E ; G \rightrightarrows M)$ and $(W \Rightarrow E ; A \Rightarrow M)$ for these data. We say that a derivation of $\mathcal{W} \rightarrow G$ is multiplicative if it generates a flow by VB-groupoid automorphisms. Similarly, a derivation of $W \rightarrow A$ is infinitesimally multiplicative (IM) if it generates a flow by VB-algebroid automorphisms. It immediately follows that if the Lie functor maps $(\mathcal{W} \rightrightarrows E ; G \rightrightarrows M)$ to $(W \Rightarrow E ; A \Rightarrow M)$, then it also maps multiplicative derivations of $\mathcal{W} \rightarrow G$ to IM derivations of $W \rightarrow A$. Additionally, if $G \rightrightarrows M$ is source simply connected (hence $\mathcal{W} \rightrightarrows E$ is source simply connected $[7]$ ) then the Lie functor establishes a one-to-one correspondence between multiplicative derivations of $\mathcal{W} \rightarrow G$ and IM derivations of $W \rightarrow A$. Our main result in this section is an algebraic description of IM derivations of a VB-algebroid ( $W \Rightarrow E ; A \Rightarrow M$ ) (Theorem 2.24) which parallels a result by Mackenzie and Xu in [47] stating that there is a one-to-one correspondence between IM vector fields on a Lie algebroid and Lie algebroid derivations. We also prove that multiplicative derivations (resp. IM derivations) are 1-cocycles in a certain linear subcomplex of the deformation complex of the Lie groupoid $\mathcal{W}$ (resp. Lie algebroid $W$ ) (Proposition 2.28, resp. Corollary 2.32).

Derivations of a vector bundle $E \rightarrow M$ are sections of the gauge algebroid $\mathrm{D} E \rightarrow M$ (see Section 2.1). Unfortunately, unlike the tangent bundle of a groupoid (resp. algebroid), the gauge algebroid of a VB-groupoid (resp. algebroid) is not a groupoid itself in general. Hence, unlike multiplicative vector fields [47], multiplicative (resp. IM) derivations of a VB-groupoid ( $\mathcal{W} \rightrightarrows E ; G \rightrightarrows M$ ) (resp. algebroid $(W \Rightarrow E ; A \Rightarrow M))$ can not be characterized, in general, as groupoid (resp. algebroid) morphisms $G \rightarrow \mathrm{DW}$ (resp. $A \rightarrow \mathrm{DW}$ ). In Section 3, we isolate a particular class of VB-groupoids (resp. algebroids) for which the associated gauge algebroid is a VB-groupoid (resp. algebroid) itself, 
namely either trivial-core or full-core VB-groupoids (resp. algebroids) and prove that in this case multiplicative (resp. IM) derivations can be characterized as morphic sections of the gauge VB-groupoid (resp. algebroid) (Theorems 3.17, 3.19). This parallels similar results for multiplicative (resp. IM) vector fields and the tangent VB-groupoid (resp. algebroid) [47]. Finally, Section 4 contains an application of the results of Section 3 to representations up to homotopy.

\section{Preliminaries}

\section{$1.1 \quad$ VB-groupoids}

In this section, we recall basic aspects of the theory of VB-groupoids and VB-algebroids. We refer to $7,27,28,46$ for more details. Given a Lie groupoid $G \rightrightarrows M$, we usually denote by $s, t: G \rightarrow M$ the source and target respectively, by $u: M \rightarrow G$ the unit, by $G^{(2)}=G_{s} \times{ }_{t} G \subset G \times G$ the submanifold of composable arrows, by $m: G^{(2)} \rightarrow G$ the multiplication, and by $i: G \rightarrow G$ the inversion. Alternatively, we denote the unit by $x \mapsto 1_{x}$, the multiplication by $(g, h) \mapsto g h$, and the inversion by $g \mapsto g^{-1}$. For $g \in G$, we write $g: x_{1} \rightarrow x_{2}$ to mean that $s(g)=x_{1}$ and $t(g)=x_{2}$. Finally, we identify $M$ with its image under $u$. Consider a diagram

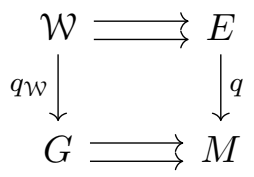

where the rows are Lie groupoids and the columns are vector bundles. We denote by $\tilde{s}, \tilde{t}, \tilde{u}, \tilde{m}, \tilde{i}$ the structure maps of the top row (source, target, unit, multiplication, inversion respectively) and by $s, t, u, m, i$ the structure maps of the bottom row. Additionally, we denote by $+\mathcal{W}: \mathcal{W} \times_{G} \mathcal{W} \rightarrow \mathcal{W}$ and $+_{E}: E \times_{M} E \rightarrow E$ the additions, by $\lambda \cdot \mathcal{W}: \mathcal{W} \rightarrow \mathcal{W}$ and $\lambda_{E}: E \rightarrow E$ the scalar multiplications by $\lambda \in \mathbb{R}$, by $0_{\mathcal{W}}: G \rightarrow \mathcal{W}$ and $0_{E}: M \rightarrow E$ the zero sections. Notice that throughout this paper, for any vector bundle $q: V \rightarrow N$, we will denote by $s_{x}$ the value of a section $s \in \Gamma(V)$ at a point $x \in N$.

Definition 1.1 (VB-groupoid) A VB-groupoid is a vector bundle object in the category of Lie groupoids, i.e. a diagram like (1.1) such that $\left(q_{\mathcal{W}}, q\right)$ and $\left(+_{\mathcal{W}},+_{E}\right)$ are Lie groupoid morphisms. Equivalently, a VB-groupoid is a Lie groupoid object in the category of vector bundles, i.e. a diagram (1.1) such that $(\tilde{s}, s),(\tilde{t}, t)$ and $(\tilde{m}, m)$ are vector bundle morphisms.

It follows from the definition, that $(\lambda \cdot \mathcal{W}, \lambda \cdot E)$ and $\left(0_{\mathcal{W}}, 0_{E}\right)$ are also Lie groupoid morphisms, for all $\lambda \in \mathbb{R}$ and that $(\tilde{u}, u)$ and $(\tilde{i}, i)$ are also vector bundle morphisms. When there is no risk of ambiguity, we simply denote by ( $\mathcal{W} \rightrightarrows E ; G \rightrightarrows M)$ (or even just $\mathcal{W}$ ) a VB-groupoid, thus understanding all structure maps.

Definition 1.2 (VB-groupoid morphism) Let $\left(\mathcal{W}_{1} \rightrightarrows E_{1} ; G_{1} \rightrightarrows M_{1}\right)$ and $\left(\mathcal{W}_{2} \rightrightarrows E_{2} ; G_{2} \rightrightarrows M_{2}\right)$ be VB-groupoids. A morphism of VB-groupoids

$$
\left(F_{\mathcal{W}}, \tilde{F} ; F_{G}, F\right):\left(\mathcal{W}_{1} \rightrightarrows E_{1} ; G_{1} \rightrightarrows M_{1}\right) \rightarrow\left(\mathcal{W}_{2} \rightrightarrows E_{2} ; G_{2} \rightrightarrows M_{2}\right)
$$


is a (commutative) diagram

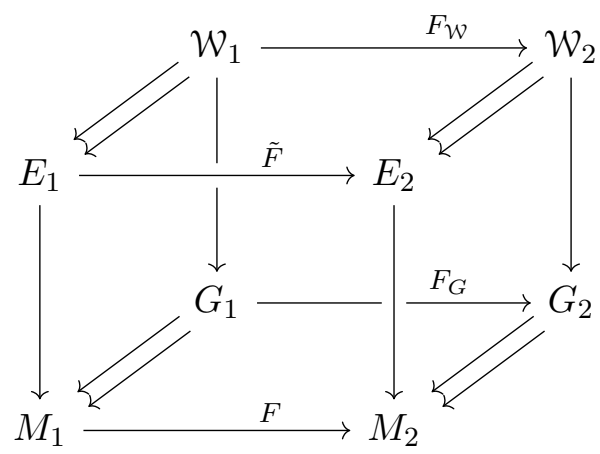

such that the following conditions are satisfied:

i.) $\left(F_{\mathcal{W}}, \tilde{F}\right)$ and $\left(F_{G}, F\right)$ are morphisms of groupoids,

ii.) $\left(F_{\mathcal{W}}, F_{G}\right)$ and $(\tilde{F}, F)$ are morphisms of vector bundles.

Notice that the map $F_{\mathcal{W}}$ in 1.2 determines the morphism completely. Accordingly, we also say that $F_{\mathcal{W}}: \mathcal{W}_{1} \rightarrow \mathcal{W}_{2}$ is a morphism of VB-groupoids.

The (right) core of a VB-groupoid $(\mathcal{W} \rightrightarrows E ; G \rightrightarrows M)$ is the vector bundle $C \rightarrow M$ defined by setting

$$
C:=\left\{\omega \in \mathcal{W}: \tilde{s}(\omega)=0, \text { and } q_{\mathcal{W}}(\omega)=1\right\} .
$$

In other words, $C=u^{*}(\operatorname{ker} \tilde{s})=\left.\operatorname{ker} \tilde{s}\right|_{M}$ is the pull-back of the vector subbundle $\operatorname{ker} \tilde{s} \subset \mathcal{W}$ along the unit. There is a similar notion of left core where the target is in place of the source. It turns out that the right and the left cores are canonically isomorphic as vector bundles. Hence they are manifestations of a unique vector bundle which can be safely referred to as the core.

The dual vector bundle $\mathcal{W}^{*}$ is a Lie groupoid over $C^{*}$, and, actually, $\left(\mathcal{W}^{*} \rightrightarrows C^{*} ; G \rightrightarrows M\right)$ is a VB-groupoid, the dual VB-groupoid of $(\mathcal{W} \rightrightarrows E ; G \rightrightarrows M)$. Additionally the VB-groupoids $\mathcal{W}$ and $\mathcal{W}^{* *}$ are canonically isomorphic. We refer to 46 for more details on this construction. Here we only notice that given a VB-groupoid isomorphism $F_{\mathcal{W}}: \mathcal{W}_{1} \rightarrow \mathcal{W}_{2}$, its transpose $F_{\mathcal{W}}^{*}: \mathcal{W}_{2}^{*} \rightarrow \mathcal{W}_{1}^{*}$ is a VBgroupoid isomorphism as well. In particular, VB-groupoid automorphisms $\mathcal{W} \rightarrow \mathcal{W}$ are equivalent to VB-groupoid automorphisms $\mathcal{W}^{*} \rightarrow \mathcal{W}^{*}$.

Example 1.3 (Tangent VB-groupoid) Let $G \rightrightarrows M$ be a Lie groupoid with Lie algebroid $A$. Then $(T G, T M ; G, M)$ is a VB-groupoid, called the tangent VB-groupoid to $G$, in a natural way: the

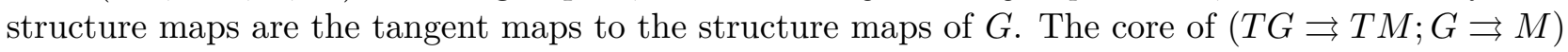
is $A$.

\section{$1.2 \quad$ VB-algebroids}

We now turn to the infinitesimal counterpart of VB-groupoids, the so-called VB-algebroids. First of all, given a Lie algebroid $A \Rightarrow M$, we usually denote by $\rho: A \rightarrow T M$ the anchor and by $[-,-]: \Gamma(A) \times \Gamma(A) \rightarrow \Gamma(A)$ the Lie bracket. Consider a diagram

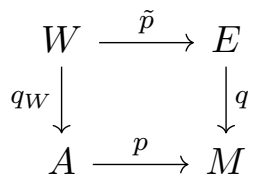

where the rows and the columns are vector bundles. We denote by $+_{W, A}: W \times_{A} W \rightarrow W,+_{W, E}$ : $W \times_{E} W \rightarrow W$ the additions, by $\lambda \cdot{ }_{W, A}: W \rightarrow W$ and $\lambda \cdot_{W, E}: W \rightarrow W$ the scalar multiplications by 
$\lambda \in \mathbb{R}$ (in the fibers over $A$ and $E$ respectively), and by $0_{W, A}: A \rightarrow W$ and $0_{W, E}: E \rightarrow W$ the zero sections. If the maps $\left(q_{W}, q\right)$ and $\left(+_{W, A},+_{E}\right)$ are vector bundle morphisms, or, equivalently $(\tilde{p}, p)$ and $\left(+_{W, E},+_{A}\right)$ are vector bundle morphisms, then (1.5) is a double vector bundle [46. Chapter 9], denoted $(W \rightarrow E ; A \rightarrow M)$, and it follows that $(\lambda \cdot W, A, \lambda \cdot E),\left(0_{W, A}, 0_{E}\right),(\lambda \cdot W, E, \lambda \cdot A)$ and $\left(0_{W, E}, 0_{A}\right)$ are also vector bundle morphisms.

Remark 1.4 (Core and linear sections of a double vector bundle) Recall that the core of a double vector bundle $(1.5)$ is the vector bundle

$$
C:=\operatorname{ker} \tilde{p} \cap \operatorname{ker} q_{W} \rightarrow M \text {. }
$$

Vector bundle $W \rightarrow E$ has two distinguished classes of sections:

i.) core sections: for every section $\chi$ of the core $C \rightarrow M$, there is a section $\hat{\chi}$ of $W \rightarrow E$ defined by:

$$
\widehat{\chi}_{e}:=\left(0_{W, E}\right)_{e}+W, A \chi_{q(e)}
$$

for all $e \in E$.

ii.) linear sections: they are sections $\tilde{a}$ of $W \rightarrow E$ such that $(\tilde{a}, a)$ is a vector bundle morphism for a (necessarily unique) section $a$ of $A$.

We denote by $\Gamma(W, E)$ the space of all sections of $W \rightarrow E$, by $\Gamma_{c}(W, E)$ the subspace of core sections and by $\Gamma_{\ell}(W, E)$ the subspace of linear sections. Linear and core sections generate $\Gamma(W, E)$ as a $C^{\infty}(E)$-module. Actually, linear sections alone generate $\Gamma(W, E)$ away from the (image of) the zero section of $E$, i.e., for every point $e \in E \backslash 0_{E}(M)$, there exists an open neighborhood $\mathcal{U}$ of $e$ such that the $C^{\infty}(\mathcal{U})$-module of local sections of $W \rightarrow E$ defined on $\mathcal{U}$ is spanned by linear sections. This is easily seen in local coordinates adapted to the double vector bundle structure.

From now on, we also assume that the rows of 1.5 possess Lie algebroid structures $W \Rightarrow E$, and $A \Rightarrow M$. We denote by $\tilde{\rho}: W \rightarrow T E$ the anchor.

Definition 1.5 (VB-algebroid) A VB-algebroid is a vector bundle object in the category of Lie algebroids, i.e. a diagram like (1.5) such that $\left(q_{W}, q\right)$ and $\left(+_{W, A},+_{E}\right)$ are Lie algebroid morphisms. Equivalently, a VB-algebroid is a Lie algebroid object in the category of vector bundles, i.e. a diagram (1.5) such that $(\tilde{p}, p),\left(+_{W, E},+_{A}\right)$ and $(\tilde{\rho}, \rho)$ are morphisms of vector bundles, and the Lie bracket in $\Gamma(W, E)$ satisfies the following linearity conditions:

i.) $\left[\Gamma_{\ell}(W, E), \Gamma_{\ell}(W, E)\right] \subset \Gamma_{\ell}(W, E)$,

ii.) $\left[\Gamma_{\ell}(W, E), \Gamma_{c}(W, E)\right] \subset \Gamma_{c}(W, E)$,

iii.) $\left[\Gamma_{c}(W, E), \Gamma_{c}(W, E)\right]=0$.

We denote simply by ( $W \Rightarrow E ; A \Rightarrow M)$ a VB-algebroid and when there is no risk of ambiguity we only use $W$.

Definition 1.6 (VB-algebroid morphism) Let $\left(W_{1} \Rightarrow E_{1} ; A_{1} \Rightarrow M_{1}\right)$ and $\left(W_{2} \Rightarrow E_{2} ; A_{2} \Rightarrow M_{2}\right)$ be VB-algebroids. A morphism of VB-algebroids

$$
\left(F_{W}, \tilde{F} ; F_{A}, F\right):\left(W_{1} \Rightarrow E_{1} ; A_{1} \Rightarrow M_{1}\right) \rightarrow\left(W_{2} \Rightarrow E_{2} ; A_{2} \Rightarrow M_{2}\right)
$$


is a (commutative) diagram

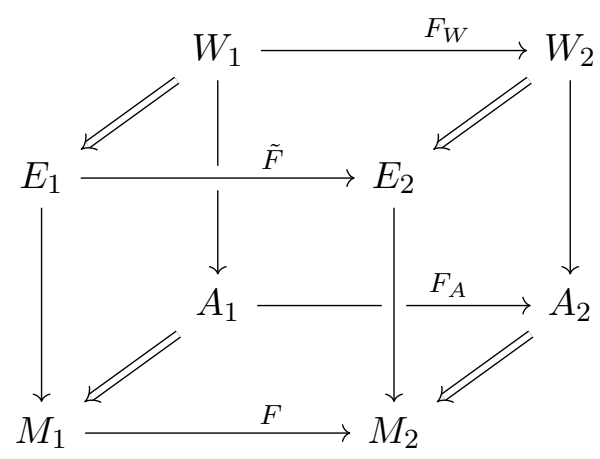

such that the following conditions are satisfied:

i.) $\left(F_{W}, \tilde{F}\right)$ and $\left(F_{A}, F\right)$ are morphisms of Lie algebroids

ii.) $\left(F_{W}, F_{A}\right)$ and $(\tilde{F}, F)$ are morphisms of vector bundles.

The map $F_{W}$ in 1.9 determines the morphism completely. Accordingly, we sometimes say that $F_{W}: W_{1} \rightarrow W_{2}$ is a morphism of VB-algebroids.

Similarly as VB-groupoids, VB-algebroids can be dualized. Let $(W \Rightarrow E ; A \Rightarrow M)$ be a VBalgebroid with core $C \rightarrow M$. Then the dual $W^{*}$ of the vector bundle $W \rightarrow A$ is a VB-algebroid and $\left(W^{*} \Rightarrow C^{*} ; A \Rightarrow M\right)$ is a VB-algebroid, called the dual VB-algebroid of ( $\left.W \Rightarrow E ; A \Rightarrow M\right)$. Additionally the VB-algebroids $W$ and $W^{* *}$ are canonically isomorphic. We refer to [46] again for more details. Here we only notice that similarly as for VB-groupoids, given a VB-algebroid isomorphism $F_{W}: W_{1} \rightarrow W_{2}$, its transpose $F_{W}^{*}: W_{2}^{*} \rightarrow W_{1}^{*}$ is a VB-algebroid isomorphism as well. Hence VB-algebroid automorphisms $W \rightarrow W$ are equivalent to VB-algebroid automorphisms $W^{*} \rightarrow W^{*}$.

Example 1.7 Let $E \rightarrow M$ be a vector bundle. Then $(T E \Rightarrow E ; T M \Rightarrow M)$ is a VB-algebroid in the obvious way.

Example 1.8 (Tangent VB-algebroid) Let $A \Rightarrow M$ be a Lie algebroid. Then $(T A \Rightarrow T M ; A \Rightarrow$ $M)$ is a VB-algebroid, called the tangent $V B$-algebroid to $A$, in a natural way. The projection $T A \rightarrow T M$ and the anchor $\tilde{\rho}: T A \rightarrow T T M$ are the tangent map to the projection $A \rightarrow M$, and the composition of the tangent map to the anchor $\rho: A \rightarrow T M$, followed by the canonical involution $T T M \rightarrow T T M$ (see, e.g., [46, Section 9.6]). The Lie bracket

$$
[-,-]: \Gamma(T A, T M) \times \Gamma(T A, T M) \rightarrow \Gamma(T A, T M)
$$

is uniquely defined by its action on certain distinguished sections, as follows. First notice that $(T A \rightarrow T M ; A \rightarrow M)$ is a double vector bundle with core canonically isomorphic to $A$ itself. The isomorphism identifies the tangent space to a fiber of $A \rightarrow M$ at the origin, with the fiber itself. Now, consider the following distinguished classes of sections of $T A \rightarrow T M$ :

i.) core sections,

ii.) linear sections of the form $\mathrm{d} a: T M \rightarrow T A$ for some $a \in \Gamma(A)$.

We denote by $\widehat{a}_{T}: T M \rightarrow T A$ the core section corresponding to $a \in \Gamma(A)$, seen as a section of the core. We have

$$
\left(\widehat{a}_{T}\right)_{v}=\left(\mathrm{d} 0_{A}\right)(v)+a_{\left(0_{A}\right)_{x}}^{\uparrow}
$$


for all $v \in T_{x} M, x \in M$, where $a^{\uparrow} \in \mathfrak{X}(A)$ is the vertical lift of $a$ (see also Remark 2.1). Then bracket 1.10 is completely determined by

$$
\begin{aligned}
& {[\mathrm{d} a, \mathrm{~d} b]=\mathrm{d}[a, b],} \\
& {\left[\mathrm{d} a, \widehat{b}_{T}\right]=\left[\widehat{a, b}_{T},\right.} \\
& {\left[\widehat{a}_{T}, \widehat{b}_{T}\right]=0,}
\end{aligned}
$$

for all $a, b \in \Gamma(A)$. Finally, notice that the VB-algebroid structures on $(T A \Rightarrow A ; T M \Rightarrow M)$ and $(T A \Rightarrow T M ; A \Rightarrow M)$ are compatible in the sense that they provide an instance of a double Lie algebroid 44,45 .

Remark 1.9 (VB-groupoids/algebroids and homogeneity structures) There is an alternative definition of a VB-groupoid/algebroid that is often useful in practice. Namely, a vector bundle is the same as a manifold $E$ equipped with a regular homogeneity structure, i.e. a regular action $h: \mathbb{R} \times E \rightarrow E$, written $(\lambda, e) \mapsto h_{\lambda}(e)$, of the monoid $(\mathbb{R}, \cdot)$. The base manifold is then $M=h_{0}(E)$ and the projection $E \rightarrow M$ is $h_{0}$. The infinitesimal generator of $h_{\exp \lambda}$ is the Euler vector field on $E$. We will not explain here the word regular: we refer to [26] for more details (see also 7 , Definition 2.1.1]). Similarly, a VB-groupoid (resp. algebroid) is equivalent to a groupoid $\mathcal{W} \rightrightarrows E$ (resp. algebroid $W \rightarrow E$ ) equipped with a regular action $h: \mathbb{R} \times \mathcal{W} \rightarrow \mathcal{W}$ (resp. $h: \mathbb{R} \times W \rightarrow W$ ) of the monoid $(\mathbb{R}, \cdot)$ with the additional property that $h_{\lambda}: \mathcal{W} \rightarrow \mathcal{W}$ (resp. $h_{\lambda}: W \rightarrow W$ ) is a Lie groupoid (resp. algebroid) morphism for all $t$. In particular, a diagram like (1.1) is a VB-groupoid if and only if $\lambda \cdot \mathcal{W}: \mathcal{W} \rightarrow \mathcal{W}$ (multiplication by $\lambda$ in the fibers of $\mathcal{W} \rightarrow G$ ) is a groupoid morphism for all $\lambda$, and similarly for VB-algebroids. Notice that using this point of view a diagram like (1.2) (resp. (1.8) is a morphism if and only if $F_{\mathcal{W}}$ (resp. $F_{W}$ ) commutes with the homogeneity structures.

\subsection{Differentiation and integration}

We conclude this section discussing briefly differentiation and integration issues. We refer to [7] for details. Denote by Lie the Lie functor mapping Lie groupoids to Lie algebroids (and Lie groupoid morphisms to Lie algebroid morphisms). Let ( $\mathcal{W} \rightrightarrows E ; G \rightrightarrows M)$ be a VB-groupoid. We denote by $W=\operatorname{Lie}(\mathcal{W})$ the Lie algebroid of $\mathcal{W}$, and by $A=\operatorname{Lie}(G)$ the Lie algebroid of $G$. Then $(W \Rightarrow$ $E ; A \Rightarrow M$ ) is a VB-algebroid in a natural (and obvious) way, and it is called the VB-algebroid of $(\mathcal{W} \rightrightarrows E ; G \rightrightarrows M)$. We also denote the latter by $\operatorname{Lie}(\mathcal{W} \rightrightarrows E ; G \rightrightarrows M)$. Additionally,

i.) $(\mathcal{W} \rightrightarrows E ; G \rightrightarrows M)$ and $(W \Rightarrow E ; A \Rightarrow M)$ have canonically isomorphic cores (we denote both by $C$ ), and

ii.) $\operatorname{Lie}\left(\mathcal{W}^{*} \rightrightarrows C^{*} ; G \rightrightarrows M\right) \cong\left(W^{*} \Rightarrow C^{*} ; A \Rightarrow M\right)$ canonically.

Similarly, the Lie functor maps morphisms of VB-groupoids to morphisms of VB-algebroids. Conversely, let $(W \Rightarrow E ; A \Rightarrow M)$ be a VB-algebroid. If $W$ is integrable and $\mathcal{W} \rightrightarrows E$ is its source simply connected integration, then 1) $A$ is integrable as well, and, denoted by $G \rightrightarrows M$ its source simply connected integration, 2$)(\mathcal{W} \rightrightarrows E ; G \rightrightarrows M)$ is a VB-groupoid in a natural way. Finally, if $\left(\mathcal{W}^{\prime} \rightrightarrows E^{\prime} ; G^{\prime} \rightrightarrows M^{\prime}\right)$ is another VB-groupoid, $\left(W^{\prime} \Rightarrow E ; A^{\prime} \Rightarrow M^{\prime}\right)$ is its VB-algebroid and $F_{W}: W \rightarrow W^{\prime}$ is a morphism of VB-algebroids, then $F_{W}$ integrates to a unique morphism $F_{\mathcal{W}}: \mathcal{W} \rightarrow \mathcal{W}^{\prime}$ of VB-groupoids.

Remark 1.10 Let $G \rightrightarrows M$ be a Lie groupoid and let $A=\operatorname{Lie}(G)$ be its Lie algebroid. Then the VBalgebroid of the tangent VB-groupoid to $G$, is (canonically isomorphic to) the tangent VB-algebroid to $A$ : $\operatorname{Lie}(T G \rightrightarrows T M ; G \rightrightarrows M) \cong(T A \Rightarrow T M ; A \Rightarrow M)$. 


\section{Infinitesimal automorphisms of VB-groupoids and algebroids}

\subsection{Derivations}

Let $E \rightarrow M$ be a vector bundle, and let $x \in M$. A derivation at $x$ is an $\mathbb{R}$-linear map $\Delta: \Gamma(E) \rightarrow E_{x}$ such that

$$
\Delta(f e)=v(f) e_{x}+f(x) \Delta(e)
$$

for all $f \in C^{\infty}(M), e \in \Gamma(E)$ and some (necessarily unique) tangent vector $v \in T_{x} M$. We also call $v$ the symbol of $\Delta$, and denote it by $\sigma(\Delta)$.

It is useful to mention that there is an alternative (more geometric) description of derivations at $x$. Let $\varepsilon \mapsto \gamma(\varepsilon)$ be a smooth curve in $M$ such that $\gamma(0)=x$ and let $\varepsilon \mapsto \gamma_{E}(\varepsilon): E_{x} \rightarrow E_{\gamma(\varepsilon)}$ be a smooth curve of linear isomorphisms (covering $\gamma$ ) such that $\gamma_{E}(0)=\operatorname{id}_{E_{x}}$. The velocity of $\gamma_{E}$ is the following derivation at $x$

$$
\left.\frac{d}{d \varepsilon}\right|_{\varepsilon=0} \gamma_{E}(\varepsilon): \Gamma(E) \rightarrow E_{x}, \quad e \mapsto\left(\left.\frac{d}{d \varepsilon}\right|_{\varepsilon=0} \gamma_{E}(\varepsilon)\right) e:=\left.\frac{d}{d \varepsilon}\right|_{\varepsilon=0} \gamma_{E}(\varepsilon)^{-1} e_{\gamma(\varepsilon)} .
$$

Additionally, the symbol of $\left.\frac{d}{d \varepsilon}\right|_{\varepsilon=0} \gamma_{E}(\varepsilon)$ is $\left.\frac{d}{d \varepsilon}\right|_{\varepsilon=0} \gamma(\varepsilon)$. Every derivation at $x$ arises in this way.

Derivations of $E$ at $x$ form a vector space denoted $\mathrm{D}_{x} E$. If we let $x$ vary, we get a vector bundle $\mathrm{D} E \rightarrow M$. Sections of $\mathrm{D} E$ identify with derivations of $E$, i.e. $\mathbb{R}$-linear operators $\Delta: \Gamma(E) \rightarrow \Gamma(E)$ such that

$$
\Delta(f e)=X(f) e+f \Delta(e)
$$

for all $f \in C^{\infty}(M), e \in \Gamma(E)$, and some (necessarily unique) vector field $X \in \mathfrak{X}(M)$. We call $X$ the symbol of $\Delta$ and denote it by $\sigma(\Delta)$ or $\sigma_{\Delta}$. In the literature, derivations of a vector bundle are referred to with different names, including der-operators [20] and covariant differential operators [46]. From now on we identify sections of $\mathrm{D} E \rightarrow M$ and derivations of $E$ in the obvious way. In particular, we denote by $\Gamma(D E)$ the space of derivations of $E$.

The vector bundle $\mathrm{D} E$ carries a structure of Lie algebroid: the Atiyah or gauge algebroid of $E$. The Lie bracket of two derivations is the commutator and the anchor is the symbol $\sigma: \mathrm{D} E \rightarrow T M$, $\Delta \mapsto \sigma(\Delta)$. Notice that a representation of a Lie algebroid $A \rightarrow M$ can be regarded as a vector bundle $E \rightarrow M$ equipped with a Lie algebroid morphism $A \rightarrow \mathrm{D} E$. The kernel of the symbol $\sigma: \mathrm{D} E \rightarrow T M$ is the endomorphism bundle of $E$. Accordingly, there is a short exact sequence of vector bundles:

$$
0 \longrightarrow \text { End } E \longrightarrow \mathrm{DE} \stackrel{\sigma}{\longrightarrow} T M \longrightarrow 0 .
$$

The correspondence $E \mapsto \mathrm{D} E$ is functorial. To see this, first recall that a vector bundle morphism is called regular if it is an isomorphism on fibers. A regular vector bundle morphism $\phi_{E}: E_{N} \rightarrow E$ covering a smooth map $\phi: N \rightarrow M$ allows us to pull-back sections of $E$ to sections of $E_{N}$. Specifically, let $e \in \Gamma(E)$. Its pull-back along $\phi_{E}$ is the section $\phi_{E}^{*} e \in \Gamma\left(E_{N}\right)$ defined by

$$
\left(\phi_{E}^{*} e\right)_{x}=\left.\phi_{E}\right|_{\left(E_{N}\right)_{x}} ^{-1}\left(e_{\phi(x)}\right),
$$

for all $x \in N$. Notice that we can use a regular vector bundle morphism $\phi_{E}: E_{N} \rightarrow E$ covering $\phi: N \rightarrow M$ to identify $E_{N}$ with the pull-back bundle $\phi^{*} E=N \times_{M} E$ in a canonical way. In the following, we always make use of this identification. In particular, we denote $\phi^{*} E=E_{N}$, and denote by $\phi_{E}: \phi^{*} E \rightarrow E$ the canonical map. Sometimes, if there is no risk of confusion, we also denote simply by $\phi^{*} e$ the pull-back section $\phi_{E}^{*} e$. Now, let $E \rightarrow M$ and $E_{N} \rightarrow N$ be vector bundles, and let $\phi_{E}: E_{N} \rightarrow E$ be a regular vector bundle morphism, covering the smooth map $\phi: N \rightarrow M$. Morphism $\phi_{E}$ determines a (generically non regular) vector bundle morphism

$$
\mathrm{D} \phi_{E}: \mathrm{D} E_{N} \rightarrow \mathrm{D} E
$$


covering $\phi: N \rightarrow M$ by

$$
\mathrm{D} \phi_{E}(\Delta)(e)=\left(\phi_{E} \circ \Delta\right)\left(\phi^{*} e\right)
$$

for all $\Delta \in \mathrm{D} E_{N}$ and $e \in \Gamma(E)$. If

$$
\Delta=\left.\frac{d}{d \varepsilon}\right|_{\varepsilon=0} \gamma_{E}(\varepsilon)
$$

for some curve $\varepsilon \mapsto \gamma_{E}(\varepsilon)$ of fiber isomorphisms, then

$$
\mathrm{D} \phi_{E}(\Delta)=\left.\left.\frac{d}{d \varepsilon}\right|_{\varepsilon=0} \phi_{E} \circ \gamma_{E}(\varepsilon) \circ \phi_{E}\right|_{\left(E_{N}\right)_{x}} ^{-1},
$$

where $x$ is the base point of $\Delta$, i.e. $\Delta \in \mathrm{D}_{x} E_{N}$.

Clearly, diagram

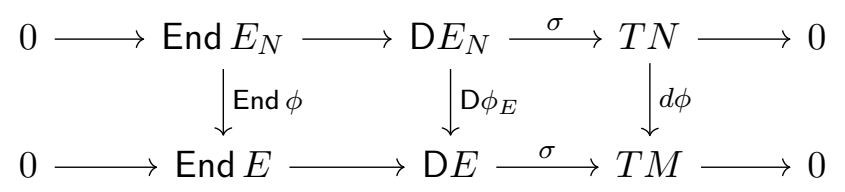

commutes. Here End $\phi=\phi_{\text {End } E}:$ End $E \rightarrow$ End $E$ is the obvious regular vector bundle morphism (covering $\phi$ ). Even more, $\mathrm{D} \phi_{E}: \mathrm{D} E_{N} \rightarrow \mathrm{D} E$ is actually a morphism of Lie algebroids (covering $\phi$ ). We will often denote $\mathrm{D} \phi_{E}$ simply by $\mathrm{D} \phi$, if there is no risk of confusion.

It is easy to see that the rightmost square in the diagram $(2.10)$ is a pull-back diagram. In particular, $\mathrm{D} E_{N}$ is canonically isomorphic to a pull-back bundle:

$$
\mathrm{D} E_{N} \cong T N_{d \phi} \times_{\sigma} \mathrm{D} E .
$$

More precisely, $\mathrm{D} E_{N}$ is the pull-back Lie algebroid of $\mathrm{D} E$ along the smooth map $\phi: N \rightarrow M$ (which makes sense, because, as the anchor $\sigma: \mathrm{D} E \rightarrow T M$ is surjective, $\phi$ is automatically transverse to it).

The case when $\phi: N \rightarrow M$ is the inclusion of a submanifold is of a special interest. In this case $E_{N}=\left.E\right|_{N}$ and $\mathrm{D} \phi: \mathrm{D} E_{N} \rightarrow \mathrm{D} E$ is an embedding whose image consists of derivations of $E$ with symbol tangent to $N$.

There is another way to understand derivations of a vector bundle $p: E \rightarrow M$. They can be regarded as infinitesimal automorphisms of $E$. A vector field $\tilde{X}$ on the total space $E$ of the vector bundle $E \rightarrow M$ is an infinitesimal automorphism if it generates a flow by vector bundle automorphisms. In particular, if $\tilde{X}$ is an infinitesimal automorphism of $E$, then it projects onto a vector field $p_{*} \tilde{X}$ on $M$.

Remark 2.1 There are several characterizations of infinitesimal automorphisms of the vector bundle $E \rightarrow M$ that we now discuss. First of all, let $h: \mathbb{R} \times E \rightarrow E$ be the (regular) homogeneity structure encoding the vector bundle structure (see Remark 1.9 ), and let $\mathcal{E}_{E}$ be the infinitesimal generator of $h_{\exp \lambda}$, i.e. the Euler vector field on $E$. Take $\tilde{X}$ a vector field on $E$. The following conditions are equivalent:

i.) $\tilde{X}$ is an infinitesimal automorphism;

ii.) for every fiber-wise linear function $\varphi$ on $E$, the Lie derivative $\tilde{X}(\varphi)$ is fiber-wise linear;

iii.) for every fiber-wise constant vector field $Y$ on $E$, the commutator $[\tilde{X}, Y]$ is fiber-wise constant;

iv.) $\left(h_{\lambda}\right)_{*} X=X$ for all $\lambda \neq 0$

v.) $\left[\mathcal{E}_{E}, X\right]=0$; 
vi.) when regarded as a section of $T E \rightarrow E, \tilde{X}$ is a linear section of (the top row) of the double vector bundle $(T E \rightarrow E ; T M \rightarrow M)$, i.e. $\tilde{X} \in \Gamma_{\ell}(T E, E)$.

Point iii.) needs some explanations. First of all, there is a canonical one-to-one correspondence between fiber-wise linear functions on $E$ and sections of the dual vector bundle $E^{*} \rightarrow M$. Now, let $e$ be a section of $E$, and let $e^{\uparrow} \in \mathfrak{X}(E)$ be its vertical lift, i.e. $e^{\uparrow}$ is the vector field defined by

$$
e_{e_{0}}^{\uparrow}:=\left.\frac{d}{d \varepsilon}\right|_{\varepsilon=0}\left(e_{0}+\varepsilon e_{p\left(e_{0}\right)}\right)
$$

for all $e_{0} \in E$. Notice that $e^{\uparrow}$ is uniquely determined by the identity

$$
e^{\uparrow}(\varphi)=\langle\varphi, e\rangle
$$

where $\varphi \in \Gamma\left(E^{*}\right)$ is also understood as a fiber-wise linear function on $E$. A fiber-wise constant vector field on $E$ is a vector field of the form $e^{\uparrow}$.

If $\tilde{X}$ satisfies one of the above equivalent conditions we also say that $\tilde{X}$ is linear.

We conclude this remark noticing that a function $f \in C^{\infty}(E)$ is fiber-wise constant if and only if $h_{\lambda}^{*} f=f$, and it is fiber-wise linear if and only if $h_{\lambda}^{*} f=\lambda \cdot f$ for all $\lambda$. Equivalently, $f$ is fiber-wise constant if and only if $\mathcal{E}_{E}(f)=0$, and it is fiber-wise linear if and only if $\mathcal{E}_{E}(f)=f$. Similarly, a vector field $\tilde{X} \in \mathfrak{X}(E)$ is fiber-wise constant if and only if $\left(h_{\lambda}\right)_{*} \tilde{X}=\lambda \cdot \tilde{X}$, and (as already remarked) it is linear if and only if $\left(h_{\lambda}\right)_{*} \tilde{X}=\tilde{X}$, for all $\lambda \neq 0$. Equivalently, $\tilde{X}$ is fiber-wise constant if and only if $\left[\varepsilon_{E}, \tilde{X}\right]=-\tilde{X}$, and it is linear if and only if $\left[\varepsilon_{E}, \tilde{X}\right]=0$.

The following lemma is familiar to experts.

Lemma 2.2 Infinitesimal automorphisms of E correspond bijectively to derivations of E.

Proof: We report a proof for completeness. Recall that the gauge algebroids $\mathrm{D} E$ and $\mathrm{D} E^{*}$ are canonically isomorphic. The isomorphism identifies a derivation $\Delta: \Gamma(E) \rightarrow \Gamma(E)$ with derivation $\Delta^{*}$ of $E^{*}$ defined by

$$
\left\langle\Delta^{*} \varphi, e\right\rangle=\sigma(\Delta)\langle\varphi, e\rangle-\langle\varphi, \Delta e\rangle,
$$

for all $\varphi \in \Gamma\left(E^{*}\right)$ and $e \in \Gamma(E)$, where $\langle-,-\rangle: E^{*} \otimes E \rightarrow \mathbb{R}$ is the duality pairing. Here $\mathbb{R}_{M}:=$ $M \times \mathbb{R} \rightarrow M$ denotes the trivial line bundle. It is easy to see that $\Delta^{* *}=\Delta$ for all $\Delta \in \Gamma(\mathrm{D} E)$. Now, it follows from point $i$. .) in Remark 2.1 that there is a bijection between infinitesimal automorphisms and derivations of $E$, mapping $\tilde{X}$ to $\Delta_{\tilde{X}}:=\left.\tilde{X}\right|_{\Gamma\left(E^{*}\right)} ^{*}$ (where we identify fiber-wise linear functions on $E$ and sections of $\left.E^{*}\right)$.

Let $\tilde{X}$ and $\Delta_{\tilde{X}}$ be as in the proof of Lemma 2.2. Clearly, $\sigma\left(\Delta_{\tilde{X}}\right)=p_{*} \tilde{X}$. Additionally, bijection $\tilde{X} \mapsto \Delta_{\tilde{X}}$ is $C^{\infty}(M)$-linear and commutator-preserving. It follows that the flow $\left\{\tilde{\phi}_{\varepsilon}\right\}_{\varepsilon}$ of $\tilde{X}$ is uniquely determined by the following ODE

$$
\Delta e=\left.\frac{d}{d \varepsilon}\right|_{\varepsilon=0} \tilde{\phi}_{\varepsilon}^{*} e
$$

In this sense, $\Delta$ generates the flow $\left\{\tilde{\phi}_{\varepsilon}\right\}_{\varepsilon}$ of automorphisms of $E$. We denote by $\Delta \mapsto X_{\Delta}$ the inverse correspondence.

Remark 2.3 There is another way to describe the bijection $\tilde{X} \mapsto \Delta_{\tilde{X}}$, which is often useful. Namely, let $\tilde{X}$ be an infinitesimal automorphism of $E$. Then, it follows from point iii.) in Remark 2.1 that the derivation $\Delta_{\tilde{X}}$ is uniquely determined by

$$
\left(\Delta_{\tilde{X}} e\right)^{\uparrow}=\left[\tilde{X}, e^{\uparrow}\right]
$$

for all $e \in \Gamma(E)$. 
In view of the Lemma 2.2 , we can make sense of "compatibility of two derivations with respect to a, non-necessarily regular, vector bundle morphism". Namely, let $E_{1} \rightarrow M_{1}$ and $E_{2} \rightarrow M_{2}$ be vector bundles, and let $\tilde{F}: E_{1} \rightarrow E_{2}$ that be a vector bundle morphism covering a smooth map $F: M_{1} \rightarrow M_{2}$. If $\Delta_{1}$ and $\Delta_{2}$ are derivations of $E_{1}$ and $E_{2}$, respectively, we say that $\Delta_{1}$ and $\Delta_{2}$ are $\tilde{F}$-related if $X_{\Delta_{1}}$ and $X_{\Delta_{2}}$ are $\tilde{F}$-related. The latter condition can be actually restated in terms of the sole $\Delta_{1}, \Delta_{2}$. Indeed, the pull-back along $\tilde{F}$ maps fiber-wise linear functions on $E_{2}$ to fiber-wise linear functions on $E_{1}$. Accordingly, there is a well-defined map $\tilde{F}^{*}: \Gamma\left(E_{2}^{*}\right) \rightarrow \Gamma\left(E_{1}^{*}\right)$, and $\Delta_{1}$ and $\Delta_{2}$ are $\tilde{F}$-related if and only if

$$
\begin{aligned}
\Delta_{1}^{*} \circ \tilde{F}^{*} & =\tilde{F}^{*} \circ \Delta_{2}^{*}, \\
\sigma\left(\Delta_{1}\right) \circ F^{*} & =F^{*} \circ \sigma\left(\Delta_{2}\right) .
\end{aligned}
$$

If $\tilde{F}$ is a surjective submersion, then (2.18) follows directly from (2.17), $\Delta_{1}$ completely determines $\Delta_{2}$ via (2.17) and we write $\Delta_{2}=\tilde{F}_{*} \Delta_{1}$. In this case $\sigma\left(\Delta_{2}\right)=F_{*} \sigma\left(\Delta_{1}\right)$. On another hand, if $\tilde{F}: E_{1} \rightarrow E_{2}$ is the inclusion of a subbundle (over a possibly smaller base), $\sigma\left(\Delta_{2}\right)$ is tangent to $M_{1}, \Delta_{2}$ completely determines $\Delta_{1}$ and we write $\Delta_{1}=\left.\Delta_{2}\right|_{E_{1}}$. In this case $\sigma\left(\Delta_{1}\right)=\left.\sigma\left(\Delta_{2}\right)\right|_{M_{1}}$.

\subsection{Infinitesimal automorphisms and multiplicative derivations}

Recall that a vector field $X \in \mathfrak{X}(G)$ on a Lie groupoid $G \rightrightarrows M$ is multiplicative if it generates a flow by groupoid automorphisms. We begin this section adapting this notion to the case of VB-groupoids. Let $(\mathcal{W} \rightrightarrows E ; G \rightrightarrows M)$ be a VB-groupoid as in (1.1).

Definition 2.4 (Infinitesimal automorphisms of VB-groupoids) A vector field $X_{\mathcal{W}}$ on $\mathcal{W}$ is said to be an infinitesimal automorphism of ( $\mathcal{W} \rightrightarrows E ; G \rightrightarrows M)$ if it generates a flow by VB-groupoid automorphisms.

Example 2.5 Let $G \rightrightarrows M$ be a Lie groupoid and let $X$ be a multiplicative vector field on $G$. Recall that the tangent lift of $X$ is the vector field $\tilde{X}$ on $T G$ whose flow is the differential $\left\{\mathrm{d} \phi_{t}\right\}$ of the flow $\left\{\phi_{t}\right\}$ of $X$ (see, e.g., 65 ). Then, $\tilde{X}$ is an infinitesimal automorphism of the tangent VB-groupoid $(T G \rightrightarrows T M ; G \rightrightarrows M)$.

Remark 2.6 Let $X_{\mathcal{W}} \in \mathfrak{X}(\mathcal{W})$ be an infinitesimal automorphism of ( $\left.\mathcal{W} \rightrightarrows E ; G \rightrightarrows M\right)$. It immediately follows from the definition that $X_{\mathcal{W}}$ projects onto vector fields

- $X_{G}=\left(q_{\mathcal{W}}\right)_{*} X_{\mathcal{W}}$ on $G$,

- $X_{E}=\tilde{s}_{*} X_{\mathcal{W}}=\tilde{t}_{*} X_{\mathcal{W}}$ on $E$,

- $X=s_{*} X_{G}=t_{*} X_{G}=q_{*} X_{E}$ on $M$.

Again from the definition, $X_{\mathcal{W}}$ is tangent to the core $C$, and we denote by $X_{C}=\left.X_{\mathcal{W}}\right|_{C}$ the restriction. We also have that $X_{C}$ projects onto $X$. Additionally, $X_{G}$ is a multiplicative vector field on $G$ and $X_{E}\left(\right.$ resp. $\left.X_{C}\right)$ is an infinitesimal automorphism of the vector bundle $E \rightarrow M$ (resp. $C \rightarrow M$ ). From another point of view, $X_{\mathcal{W}}$ corresponds to a derivation $\Delta_{\mathcal{W}}$ of the vector bundle $\mathcal{W} \rightarrow G, X_{E}$ corresponds to a derivation $\Delta_{E}$ of the vector bundle $E \rightarrow M$, and $X_{C}$ corresponds to a derivation $\Delta_{C}$ of the vector bundle $C \rightarrow M$. Finally $\Delta_{\mathcal{W}}$ and $\Delta_{E}$ are both $\tilde{s}$ and $\tilde{t}$-related: $\Delta_{E}=\tilde{s}_{*} \Delta_{\mathcal{W}}=\tilde{t}_{*} \Delta_{\mathcal{W}}$. Similarly, $\Delta_{C}=\left.\Delta_{\mathcal{W}}\right|_{C}$.

Definition 2.7 (Multiplicative derivations) A derivation $\Delta_{\mathcal{W}}$ of $\mathcal{W} \rightarrow G$ is multiplicative if the corresponding linear vector field $X_{\Delta_{\mathcal{W}}} \in \mathfrak{X}(\mathcal{W})$ is an infinitesimal automorphism of the VB-groupoid $(\mathcal{W} \rightrightarrows E ; G \rightrightarrows M)$. 
The map $\Delta_{\mathcal{W}} \mapsto X_{\Delta_{\mathcal{W}}}$ establishes a one-to-one correspondence between multiplicative derivations and infinitesimal automorphisms of $(\mathcal{W} \rightrightarrows E ; G \rightrightarrows M)$. Consider a multiplicative derivation $\Delta_{\mathcal{W}}$ of $\mathcal{W} \rightarrow G$. It follows from Remark 2.6 that

- there is a derivation $\Delta_{E}$ of $E \rightarrow M$ such that $\Delta_{E}=\tilde{s}_{*} \Delta_{\mathcal{W}}=\tilde{t}_{*} \Delta_{\mathcal{W}}$,

- there is a derivation $\Delta_{C}$ of $C \rightarrow M$ such that $\Delta_{C}=\left.\Delta_{\mathcal{W}}\right|_{C}$,

- the symbol of $\Delta_{\mathcal{W}}$ is a multiplicative vector field on $G$ projecting on the symbols of both $\Delta_{E}$ and $\Delta_{C}$ (via both $s$ and $t$ ).

Let $(\mathcal{W} \rightrightarrows E ; G \rightrightarrows M)$ be a VB-groupoid with VB-algebroid $(W \Rightarrow E ; A \Rightarrow M)$. Take a linear section $\tilde{a}: E \rightarrow W$ covering a section $a$ of $A \rightarrow M$, and let $\overrightarrow{\tilde{a}}$ and $\overleftarrow{\tilde{a}}$ be the associated right and left invariant vector fields on $\mathcal{W}$.

Proposition 2.8 Both $\overrightarrow{\vec{a}}, \overleftarrow{a}$ are linear vector fields on $\mathcal{W}$

Proof: Consider $\overrightarrow{\vec{a}}$ first. According to Remark 2.1. point $i v$. ), it is enough to show that $\left(h_{\lambda}\right)_{*} \overrightarrow{\vec{a}}=$ $\overrightarrow{\vec{a}}$, for all $\lambda \neq 0$. So let $\omega \in \mathcal{W}$, with $\tilde{t}(\omega)=e$, and compute

$$
\left(h_{\lambda *} \overrightarrow{\vec{a}}\right)_{\omega}=\mathrm{d} h_{\lambda}\left(\mathrm{d} R_{\lambda^{-1} \omega}\left(\tilde{a}_{\lambda^{-1}} e\right)\right)=\mathrm{d} R_{\omega}\left(d h_{\lambda}\left(\tilde{a}_{\lambda^{-1}} e\right)=\mathrm{d} R_{\omega}\left(\tilde{a}_{e}\right)=\overrightarrow{\tilde{a}}_{\omega}\right.
$$

where $R$ denotes right translations in $\mathcal{W}$, and we used the linearity of $\tilde{m}$ and $\tilde{a}$ in the form of the identities $h_{\lambda} \circ R_{\lambda^{-1} \omega}=R_{\omega} \circ h_{\lambda}$ and $d h_{\lambda}\left(\tilde{a}_{\lambda^{-1}}\right)=\tilde{a}_{e}$ respectively. A similar argument (with left translations in place of right translations) shows that $h_{\lambda *} \overleftarrow{\widetilde{a}}=\overleftarrow{a}$, for all $\lambda \neq 0$, i.e. $\overleftarrow{a}$ is a linear vector field as well.

Remark 2.9 It follows from Proposition 2.8 that $\overrightarrow{\vec{a}}$ and $\overleftarrow{\tilde{a}}$ correspond to derivations of $\mathcal{W} \rightarrow G$, denoted $\vec{\Delta}_{\tilde{a}}$ and $\overleftarrow{\Delta}_{\tilde{a}}$, respectively. Moreover, $\vec{a}+\overleftarrow{a}$ is a linear vector field which is additionally multiplicative. Hence it corresponds to a multiplicative derivation of $\mathcal{W}$, namely $\vec{\Delta}_{\tilde{a}}+\overleftarrow{\Delta}_{\tilde{a}}$, also denoted $\delta \tilde{a}$, and called an internal derivation of $(\mathcal{W} \rightrightarrows E ; G \rightrightarrows M)$. By duality, $\overrightarrow{\tilde{a}}+\overleftarrow{\tilde{a}}$ does also correspond to a multiplicative derivation of $\mathcal{W}^{*}$. Similarly, a linear section of $W^{*} \rightarrow C^{*}$ determines a multiplicative derivation of both $\mathcal{W}$ and $\mathcal{W}^{*}$.

Remark 2.10 There is an important characterization of multiplicative vector fields on a Lie groupoid $G \rightrightarrows M$. Namely, a vector field $X \in \mathfrak{X}(G)$ is multiplicative if and only if, when regarded as a section $X: G \rightarrow T G$ of the tangent VB-groupoid, it is a morphism of Lie groupoids [47. This suggests the following definition. Given a VB-groupoid ( $\mathcal{W} \rightrightarrows E ; G \rightrightarrows M$ ), a section $w$ of $\mathcal{W} \rightarrow G$ is multiplicative if it is a Lie groupoid morphism. So, a vector field $X_{\mathcal{W}} \in \mathfrak{X}(\mathcal{W})$ is an infinitesimal automorphism of $(\mathcal{W} \rightrightarrows E ; G \rightrightarrows M)$ if and only if the following conditions are satisfied:

i.) it is a linear vector field with respect to the vector bundle structure $\mathcal{W} \rightarrow G$,

ii.) when regarded as a section $X_{\mathcal{W}}$ of $T \mathcal{W} \rightarrow \mathcal{W}$, it is a multiplicative section of the (tangent) VB-groupoid $(T \mathcal{W} \rightrightarrows T E ; \mathcal{W} \rightrightarrows E)$.

Yet in other words, infinitesimal automorphisms of ( $\mathcal{W} \rightrightarrows E ; G \rightrightarrows M)$ are the same as morphisms between the VB-groupoids $(\mathcal{W} \rightrightarrows E ; G \rightrightarrows M)$ and $(T \mathcal{W} \rightrightarrows T E ; T G \rightrightarrows T M)$ inverting the projection $T \mathcal{W} \rightarrow \mathcal{W}$ on the right.

Remark 2.11 A similar characterization as for multiplicative vector fields (and infinitesimal automorphism of VB-groupoids, see Remark 2.10 cannot take place, in general, for multiplicative derivations. The reason is that the gauge algebroid $\mathrm{D} \mathcal{W}$ is not a VB-groupoid itself, if $(\mathcal{W} \rightrightarrows E ; G \rightrightarrows M)$ is 
a generic VB-groupoid. In Section 3 we discuss two simple but interesting classes of VB-groupoids, those with either trivial core or trivial side vector bundle $0_{M} \rightarrow M$ (we call the latter full-core VB-groupoids). Trivial-core VB-groupoids $\left(E_{G} \rightrightarrows E ; G \rightrightarrows M\right)$ come from representations $E$ of Lie groupoids $G$ and possess the nice property that their gauge algebroid $\mathrm{D} E_{G}$ fits in a VB-groupoid $\left(\mathrm{D} E_{G} \rightrightarrows \mathrm{D} E ; G \rightrightarrows M\right)$ itself. It turns out that multiplicative derivations of a trivial-core VB-groupoid $\left(E_{G} \rightrightarrows E ; G \rightrightarrows M\right)$ are the same as multiplicative sections of $\mathrm{D} E_{G} \rightarrow G$ (see Theorem 3.17). As duality establishes a one-to-one correspondence between trivial-core and full-core VB-groupoids, similar considerations hold true for multiplicative derivation of full-core VB-groupoids.

Remark 2.12 We already have a simple and conceptually satisfactory description of infinitesimal automorphisms of a VB-groupoid ( $\mathcal{W} \rightrightarrows E ; G \rightrightarrows M)$ as morphisms between the VB-groupoids $(\mathcal{W} \rightrightarrows E ; G \rightrightarrows M)$ and $(T \mathcal{W} \rightrightarrows T E ; T G \rightrightarrows T M)$ (Remark 2.10), so the reader may wonder: why do we care so much about multiplicative derivations (Remark 2.11)? There are at least two reasons for that. First of all, the target space $T \mathcal{W}$ of an infinitesimal automorphism is an example of a triple structure: it possesses a groupoid structure $T \mathcal{W} \rightrightarrows T E$, and two compatible vector bundle structures $T \mathcal{W} \rightarrow \mathcal{W}$ and $T \mathcal{W} \rightarrow T G$. All these structures play a role when dealing with infinitesimal automorphisms of $(\mathcal{W} \rightrightarrows E ; G \rightrightarrows M)$. This could potentially lead to complications, especially in view of the fact that a detailed theory of triple structures like $T \mathcal{W}$ is still unavailable (and developing it would take us too far from our original scopes). On the other hand, derivations provide a simpler, more algebraic, and somehow minimal description of the same objects. Second, and, probably, more important, the reader should remember that this paper represents just the first step in a program aiming at studying (higher order) DOs on groupoids and algebroids. In this respect, derivations are an instance of first order DOs. Now, derivations can be seen as linear vector fields, but there is no similar description for higher order DOs. So working with derivations directly is a better training to learn how to work with generic DOs.

We now pass to VB-algebroids. First recall that a vector field $X \in \mathfrak{X}(A)$ on a Lie algebroid $A \Rightarrow M$ is infinitesimally multiplicative (IM) if it generates a flow by Lie algebroid automorphisms. Let $(W \Rightarrow E ; A \Rightarrow M)$ be a VB-algebroid. As in (1.5), we denote by $\tilde{p}: W \rightarrow E$, and $p: A \rightarrow M$ the projections.

Definition 2.13 (Infinitesimal automorphisms of VB-algebroids) A vector field $X_{W}$ on $W$ is an infinitesimal automorphism of $(W \Rightarrow E ; A \Rightarrow M)$ if it generates a flow by VB-algebroid automorphisms.

Remark 2.14 Let $X_{W} \in \mathfrak{X}(W)$ be an infinitesimal automorphism of $(W \Rightarrow E ; A \Rightarrow M)$. It immediately follows from the definition that $X_{W}$ projects onto vector fields

- $X_{A}=\left(q_{W}\right)_{*} X_{W}$ on $A$,

- $X_{E}=\tilde{p}_{*} X_{W}$ on $E$,

- $X=p_{*} X_{A}=q_{*} X_{E}$ on $M$.

Again from the definition, $X_{W}$ is tangent to the core $C$ and we denote by $X_{C}=\left.X_{W}\right|_{C}$ the restriction. We also have that $X_{C}$ projects onto $X$. Additionally, $X_{A}$ is an IM vector field on $A$, and $X_{E}$ (resp. $X_{C}$ ) is an infinitesimal automorphism of the vector bundle $E \rightarrow M$ (resp. $C \rightarrow M$ ). From another point of view, $X_{W}$ corresponds both to a derivation $\Delta_{W, A}$ of the vector bundle $W \rightarrow A$, and a derivation $\Delta_{W, E}$ of $W \rightarrow E$. In the same way, $X_{E}$ corresponds to a derivation $\Delta_{E}$ of the vector bundle $E \rightarrow M, X_{C}$ corresponds to a derivation $\Delta_{C}$ of the vector bundle $C \rightarrow M$, and $X_{A}$ corresponds to a derivation $\Delta_{A}$ of $A$ (sharing the same symbol as $\Delta_{E}$ and $\Delta_{C}$ ). Finally $\Delta_{W, A}$ and $\Delta_{E}$ are $\tilde{p}$-related, $\Delta_{C}$ agrees with the restrictions of both $\Delta_{W, A}$ and $\Delta_{W, E}$ to $C \rightarrow M$, and $\Delta_{W, E}$ and $\Delta_{A}$ are $q_{W}$-related: $\Delta_{E}=\tilde{p}_{*} \Delta_{W, A}$, 
$\Delta_{C}=\left.\Delta_{W, A}\right|_{C}=\left.\Delta_{W, E}\right|_{C}$ and $\Delta_{A}=\left(q_{W}\right)_{*} \Delta_{W, E}$. We call $\Delta_{W, A}$ the vertical derivation and $\Delta_{W, E}$ the horizontal derivation corresponding to $X_{W}$. In the following we provide a more algebraic description of both $\Delta_{E}$ and $\Delta_{C}$ in terms of $\Delta_{W, E}$ (see Theorem 2.24).

Definition 2.15 (IM derivations) A derivation $\Delta_{W, A}$ of $W \rightarrow A$ is an IM derivation if the corresponding linear vector field $X_{W} \in \mathfrak{X}(W)$ is an infinitesimal automorphism of the VB-algebroid $(W \Rightarrow E ; A \Rightarrow M)$.

Recall from [47] that if $A \Rightarrow M$ is a Lie algebroid, a Lie algebroid derivation of $A \Rightarrow M$ is a derivation $\Delta$ of $A \rightarrow M$ such that

$$
\Delta[a, b]=[\Delta a, b]+[a, \Delta b],
$$

for all $a, b \in \Gamma(A)$. It follows from 2.20 that $\Delta$ is also compatible with the anchor, i.e.

$$
\rho(\Delta a)=[\sigma(\Delta), \rho(a)]
$$

for all $a \in \Gamma(A)$. The commutator of two Lie algebroid derivations is clearly a Lie algebroid derivation as well. Now, the usual map $X \mapsto \Delta_{X}$ establishes a one-to-one, commutator-preserving, correspondence between IM vector fields on $A$ and Lie algebroid derivations of $A$ (see [47]). So, Lie algebroid derivations provide an algebraic description of IM vector fields. Notice that Lie algebroid derivations of $A$ are the same as 1-differentials of $A$ (cf. [29]).

Let $(W \Rightarrow E ; A \Rightarrow M)$ be a VB-algebroid. Take a linear section $\tilde{a}$ of $W \rightarrow E$ and consider the adjoint operator $[\tilde{a},-]$. It is a derivation of $W \rightarrow E$. Additionally, it is a Lie algebroid derivation. Thus, from the above discussion it follows that $X_{[\tilde{a},-]}$ is an infinitesimal automorphism of $W \Rightarrow E$.

Proposition 2.16 A vector field $X_{[\tilde{a},-]}$ is an infinitesimal automorphism of the VB-algebroid $(W \Rightarrow E ; A \Rightarrow M)$.

Before proving Proposition 2.16 we need some general remarks which will be useful in the next Section 2.3 as well. Let $\mathcal{E}_{W, A}$ and $\mathcal{E}_{W, E}$ be the Euler vector fields of vector bundles $W \rightarrow A$ and $W \rightarrow E$, respectively. As $W$ is a double vector bundle, $\mathcal{E}_{W, A}$ and $\mathcal{E}_{W, E}$ commute. In particular, it follows from Remark 2.1, point $v$.), that $\mathcal{E}_{W, A}$ is an infinitesimal automorphism of the vector bundle $W \rightarrow E$. Hence, it corresponds to a derivation $\Delta_{\varepsilon}$ of $W \rightarrow E$. The symbol of $\Delta_{\varepsilon}$ is $\tilde{p}_{*} \mathcal{E}_{W, A}$ and coincides with $\mathcal{E}_{E}$, the Euler vector field of $E$. The following lemma parallels the characterization of fiber-wise constant (resp. linear) functions and vector fields on a vector bundle in terms of the Euler vector field (as discussed in Remark 2.1) and can be easily proved in local coordinates adapted to the double vector bundle structure of $W$ (see e.g. [26, 27]).

Lemma 2.17 A section $w$ of $W \rightarrow E$ is a core section if and only if $\Delta_{\varepsilon} w=-w$ and it is linear if and only if $\Delta_{\varepsilon} w=0$. Additionally, there are no non-trivial sections $w$ of $W \rightarrow E$ such that $\Delta_{\varepsilon} w=-i w$ for some integer $i>1$.

Proof (of Proposition 2.16]): As the vector field $X_{[\tilde{a},-]}$ is already known to be an infinitesimal automorphism of the Lie algebroid $W \Rightarrow E$, it remains to check that it is linear with respect to the vector bundle structure $W \rightarrow A$. Now a vector field on $W$ is linear with respect to $W \rightarrow A$ if and only if it commutes with $\mathcal{E}_{W, A}$ (cf. Remark 2.1. point $v$.)). In order to see that $X_{[\tilde{a},-]}$ commutes with $\mathcal{E}_{W, A}$ it is enough to check that the operator $[\tilde{a},-]$ commutes with $\Delta_{\varepsilon}$ (cf. Lemma 2.2). In other words we have to show that the commutator $\square$ of $[\tilde{a},-]$ and $\Delta_{\varepsilon}$ vanishes. To do this it is enough to check that

i.) the symbol of $\square$ vanishes,

ii.) $\square$ vanishes on generators (over $C^{\infty}(E)$ ). 
Now, for any linear section $\tilde{a}$ of $W \rightarrow E$, the symbol of $[\tilde{a},-]$ is $\tilde{\rho}(\tilde{a})$ and it is a linear vector field on $E$. On the other hand the symbol of $\Delta$ is the Euler vector field $\mathcal{E}_{E}$ of $E$. So $\sigma(\square)=\left[\tilde{\rho}(\tilde{a}), \mathcal{E}_{E}\right]=0$. Finally, from Definition 1.5 and Lemma 2.17, $\square$ vanishes on both core and linear sections.

Remark 2.18 It follows from Proposition 2.16 that the vector field $X_{[\tilde{a},-]}$ corresponds to an IM derivation of $W \rightarrow A$, denoted $\delta \tilde{a}$ and called an internal derivation, and by duality to an IM derivation of $W^{*} \rightarrow A$. Similarly, a linear section of $W^{*} \rightarrow C^{*}$ determines an IM derivation of both $W$ and $W^{*}$.

Note that if $(W \Rightarrow E ; A \Rightarrow M)$ is the VB-algebroid of a VB-groupoid $(\mathcal{W} \rightrightarrows W ; G \rightrightarrows M)$, then for any $\tilde{a} \in \Gamma_{\ell}(W, E)$ the vector field $X_{[\tilde{a},-]} \in \mathfrak{X}(W)$ is the image of $\overrightarrow{\tilde{a}}+\overleftarrow{\tilde{a}}$ under the Lie functor. We use the same symbol $\delta \tilde{a}$ to denote the derivations corresponding to both. It will be always clear from the context if we are referring to one or the other.

Remark 2.19 Let $A \rightarrow M$ be a Lie algebroid. As proved in [47], a vector field $X \in \mathfrak{X}(A)$ is IM if and only if, when regarded as a section $X: A \rightarrow T A$ of the tangent VB-algebroid, it is a morphism of Lie algebroids. This suggests the following definition. Given a VB-algebroid ( $W \Rightarrow E ; A \Rightarrow M)$ a section $s: A \rightarrow W$ of $W \rightarrow A$ is an IM section if it is a Lie algebroid morphism. Thus, similarly as for infinitesimal automorphisms of VB-groupoids (Remark 2.10), a vector field $X_{W} \in \mathfrak{X}(W)$ is an infinitesimal automorphism of $(W \Rightarrow E ; A \Rightarrow M)$ if and only if it is a linear vector field with respect to the vector bundle structure $W \rightarrow A$, and when regarded as a section of $T W \rightarrow W$, it is an IM section of the tangent VB-algebroid ( $T W \Rightarrow T E ; W \Rightarrow E$ ). In other words, infinitesimal automorphisms of $(W \Rightarrow E ; A \Rightarrow M)$ are the same as morphisms between the VB-algebroids $(W \Rightarrow E ; A \Rightarrow M)$ and $(T W \Rightarrow T E ; T A \Rightarrow T M)$ inverting the projection $T W \rightarrow W$ on the right.

Remark 2.20 A similar characterization as for IM vector fields cannot take place, in general, for IM derivations because the gauge algebroid $\mathrm{D} W$ of $W \rightarrow A$ is not a VB-algebroid itself, when ( $W \Rightarrow$ $E ; A \Rightarrow M)$ is a generic VB-algebroid. However, it turns out that the gauge algebroid $\mathrm{D} E_{A}$ of a trivialcore VB-algebroid $\left(E_{A} \Rightarrow E ; A \Rightarrow M\right)$ (see Section 3 fits in a VB-algebroid (DE $\left.E_{A}, \Rightarrow \mathrm{D} E ; A \Rightarrow M\right)$ itself, and IM derivations of $\left(E_{A} \Rightarrow E ; A \Rightarrow M\right)$ are the same as IM sections of $\mathrm{D} E_{A} \rightarrow A$ (Theorem 3.19). Similar considerations hold true for IM derivations of trivial-core VB-algebroids.

The following proposition proves that IM derivations of VB-algebroids are the infinitesimal counterpart of multiplicative derivations of VB-groupoids. It follows immediately from Definitions 2.4 2.7. 2.13, 2.15 and the general discussion in Subsection 1.3 (see 7 for more details).

Proposition 2.21 Let $(\mathcal{W} \rightrightarrows E ; G \rightrightarrows M)$ be a VB-groupoid with VB-algebroid $(W \Rightarrow E ; A \Rightarrow M)$. The Lie functor maps infinitesimal automorphisms of $\mathcal{W}$ to infinitesimal automorphisms of $W$. If, additionally, $\mathcal{W}$ is source simply connected, then any infinitesimal automorphism of $W$ integrates to a unique infinitesimal automorphism of $\mathcal{W}$. In particular, there is a one-to-one correspondence between multiplicative derivations of $\mathcal{W}$ and IM derivations of $W$.

We conclude this subsection presenting an alternative description of IM derivations of a VB-algebroid in the same spirit as the algebraic description of IM vector fields on a Lie algebroid provided by Lie algebroid derivations.

Remark 2.22 Let $G \rightrightarrows M$ be a Lie groupoid with Lie algebroid $A \Rightarrow M$. The Lie functor maps a multiplicative vector field $X_{G}$ on $G$ to an IM vector field $X_{A}$ on $A$. In its turn $X_{A}$ corresponds to a Lie algebroid derivation $\Delta_{A}$ of $A$. It has been shown in [47, Theorem 3.9] that for every right invariant vector field $Y$ on $G,\left[X_{G}, Y\right]$ is a right invariant vector field and, more importantly, $X_{G}$ and $\Delta_{A}$ are related by the following formula:

$$
\overrightarrow{\Delta_{A} a}=\left[X_{G}, \vec{a}\right]
$$

for all $a \in \Gamma(A)$. 
Now, let $(W \Rightarrow E ; A \Rightarrow M)$ be a VB-algebroid, and let $X_{W}$ be an infinitesimal automorphism of it. The horizontal derivation $\Delta_{W, E}$ corresponding to $X_{W}$ is a Lie algebroid derivation of $W \Rightarrow E$. We want to provide an alternative description of those Lie algebroid derivations of $W \Rightarrow E$ that arise in this way. In order to do this, we need to recall some extra features of VB-algebroids.

The space $\Gamma_{\ell}(W, E)$ of linear sections of $W \rightarrow E$ (see Remark 1.4) is a $C^{\infty}(M)$-module in an obvious way. Actually, it is the module of sections of a vector bundle $A \rightarrow M$. Specifically, a point of $\widehat{A}$ over a point $x \in M$ is a pair $(a, h)$ consisting of a point $a$ of $A_{x}$ and a linear map $h: E_{x} \rightarrow W_{a}$ which inverts the projection $W_{a} \rightarrow E_{x}$ on the right. As linear sections project onto sections of $A \rightarrow M$, there is a vector bundle epimorphism $\pi: \widehat{A} \rightarrow A$. In view of the canonical splitting $\left.W\right|_{M}=C \oplus E$, linear sections of $W \rightarrow E$ taking their values in the kernel of $\pi: \widehat{A} \rightarrow A$ identify with vector bundle morphisms $E \rightarrow C$. So there is a short exact sequence

$$
0 \longrightarrow \operatorname{Hom}(E, C) \longrightarrow \widehat{A} \stackrel{\pi}{\longrightarrow} A \longrightarrow 0,
$$

and in what follows we understand the inclusion $\operatorname{Hom}(E, C) \hookrightarrow \widehat{A}$. In this regard notice that for any $\chi \in \Gamma(C)$ and $\varphi \in \Gamma\left(E^{*}\right)$ the latter inclusion identifies $\varphi \otimes \chi \in \Gamma\left(E^{*} \otimes C\right)$ with the linear section $\varphi \hat{\chi} \in \Gamma_{\ell}(W, E)$ given by the product of the fiber-wise linear function $\varphi$ and the core section $\hat{\chi}$.

The Lie bracket on $\Gamma(W, E)$ restricts to linear sections. As a consequence, there is a Lie algebroid structure $\widehat{A} \Rightarrow M$ with anchor given by $\rho \circ \pi$. This Lie algebroid is the so-called fat algebroid of $(W \Rightarrow E ; A \Rightarrow M)$ (see [27]). Vector bundle $E \rightarrow M$ carries a canonical representation of the fat algebroid, called the side representation and denoted by $\psi^{s}$. Let $\tilde{a}$ be a section of $\widehat{A}$ or, equivalently, a linear section of $W \rightarrow E$ projecting onto a section $a$ of $A \rightarrow M$. The side representation is implicitly given by

$$
\left\langle\varphi, \psi_{\tilde{a}}^{s} e\right\rangle=\rho(a)\langle\varphi, e\rangle-\langle\tilde{\rho}(\tilde{a})(\varphi), e\rangle,
$$

for all $\varphi \in \Gamma\left(E^{*}\right)$, and $e \in \Gamma(E)$. Here, as usual, $\varphi$ is also regarded as a fiber-wise linear function on $E$. Recall that given a linear section $\tilde{a}$ of $W \rightarrow E$, vector field $\tilde{\rho}(\tilde{a})$ is an infinitesimal automorphism of the vector bundle $E$, hence its Lie derivative preserves vertical lifts of sections of $E$ (see Remark 2.3 ) and

$$
\left(\psi_{\tilde{a}}^{s} e\right)^{\uparrow}=\left[\tilde{\rho}(\tilde{a}), e^{\uparrow}\right],
$$

for all $e \in \Gamma(E)$. Yet in other words, $\psi_{\tilde{a}}^{s}$ is nothing but the derivation of $E$ corresponding to the infinitesimal automorphism $\tilde{\rho}(\tilde{a})$ of $E$ (Lemma 2.2). The core $C \rightarrow M$ does also carry a representation of the fat algebroid, called the core representation and denoted by $\psi^{c}$. The core representation is implicitely given by

$$
\widehat{\psi_{\tilde{a} \chi}^{c} \chi}=[\tilde{a}, \widehat{\chi}]
$$

for all $\tilde{a} \in \Gamma(\widehat{A})$ and $\chi \in \Gamma(C)$, where $\widehat{\chi}$ denotes the core section of $W \rightarrow E$ corresponding to $\chi$. Finally, a distinguished vector bundle morphism $\partial: C \rightarrow E$, called the core-anchor, is defined by

$$
\langle\partial \chi, \varphi\rangle=\tilde{\rho}(\widehat{\chi})(\varphi)
$$

for all $\chi \in \Gamma(C)$ and $\varphi \in \Gamma\left(E^{*}\right)$. Notice that our convention on the core-anchor differs by a sign from that of [27]. There is an equivalent way to describe the core-anchor. Actually, in view of Definition 1.5 and Lemma 2.17, for any $\chi \in \Gamma(C)$, vector field $\tilde{\rho}(\widehat{\chi}) \in \mathfrak{X}(E)$ is the vertical lift of a (necessarily unique) section of $E$ (see Remark 2.3) and

$$
(\partial \chi)^{\uparrow}=\tilde{\rho}(\widehat{\chi}) .
$$

Example 2.23 Let $E \rightarrow M$ be a vector bundle, and recall that its tangent double vector bundle $(T E \rightarrow E ; T M \rightarrow M)$ is actually a VB-algebroid $(T E \Rightarrow E ; T M \Rightarrow M)$. It follows from Remark 2.1. point vi.), and Lemma 2.2, that its fat algebroid is precisely the gauge algebroid $\mathrm{D} E$ of $E$. Additionally, both the side vector bundle and the core of $(T E \Rightarrow E ; T M \Rightarrow M)$ are (canonically isomorphic to) $E$ (see also Example 1.8) and the core/side representations both agree with the tautological action of $\mathrm{D} E$ on $E$. Finally the core-anchor is just the identity id : $E \rightarrow E$. 
We now show that infinitesimal automorphisms of ( $W \Rightarrow E ; A \Rightarrow M)$ or equivalently IM derivations of $W \rightarrow A$ can be characterized in terms of certain algebraic data, in a similar way as IM vector fields on a Lie algebroid can be characterized in terms of Lie algebroid derivations. So, let $X_{W}$ be an infinitesimal automorphism of $(W \Rightarrow E ; A \Rightarrow M)$ and let $\Delta_{W, A}$ and $\Delta_{W, E}$ be its vertical and horizontal derivations respectively. Since $X_{W}$ is, in particular, an IM vector field on $W$, then $\Delta_{W, E}$ is a Lie algebroid derivation of the Lie algebroid $W \Rightarrow E$. Additionally, $X_{W}$ is also an infinitesimal automorphism of the vector bundle $W \rightarrow A$, i.e. it commutes with $\mathcal{E}_{W, A}$ (cf. Remark 2.1, point v.)). It follows that $\Delta_{W, E}$ commutes with $\Delta_{\varepsilon}$ (see Lemma 2.2). Hence, in view of Lemma 2.17, $\Delta_{W, E}$ preserves linear sections of $W \rightarrow E$ and it restricts to a derivation $\Delta_{\widehat{A}}: \Gamma(\widehat{A}) \rightarrow \Gamma(\widehat{A})$ of the fat algebroid. Obviously, $\Delta_{\widehat{A}}$ is a Lie algebroid derivation. For the same reason, $\Delta_{W, E}$ preserves core sections of $W \rightarrow E$ and determines a derivation $\Delta_{C}: \Gamma(C) \rightarrow \Gamma(C)$ of the core. Consider also the derivations $\Delta_{A}, \Delta_{E}$ of $A, E$, respectively, as in Remark 2.14 . We are now ready to state the main result in this section.

Theorem 2.24 Let $(W \Rightarrow E ; A \Rightarrow M)$ be a VB-algebroid. The assignments $X_{W} \mapsto \Delta_{W, A}$ and $X_{W} \mapsto\left(\Delta_{\widehat{A}}, \Delta_{E}, \Delta_{C}\right)$ establish one-to-one correspondences between

- infinitesimal automorphisms of $(W \Rightarrow E ; A \Rightarrow M)$,

- IM derivations of $W \rightarrow A$,

- triples $\left(\Delta_{\widehat{A}}, \Delta_{E}, \Delta_{C}\right)$ consisting of

i.) a Lie algebroid derivation $\Delta_{\widehat{A}}$ of the fat algebroid $\widehat{A}$,

ii.) a derivation $\Delta_{E}$ of $E$,

iii.) a derivation $\Delta_{C}$ of $C$,

such that

$$
\begin{gathered}
\sigma\left(\Delta_{\widehat{A}}\right)=\sigma\left(\Delta_{E}\right)=\sigma\left(\Delta_{C}\right), \\
\Delta_{\widehat{A}} \Phi=\Delta_{C} \circ \Phi-\Phi \circ \Delta_{E},
\end{gathered}
$$

for all vector bundle morphisms $\Phi: E \rightarrow C$ (also regarded as sections of $\widehat{A}$ via the inclusion $\operatorname{Hom}(E, C) \hookrightarrow \widehat{A})$, and

$$
\begin{aligned}
& \partial \circ \Delta_{C}=\Delta_{E} \circ \partial, \\
& {\left[\Delta_{E}, \psi_{\tilde{a}}^{s}\right]=\psi_{\Delta_{\widehat{A}} \tilde{a}},} \\
& {\left[\Delta_{C}, \psi_{\tilde{a}}^{c}\right]=\psi_{\Delta_{\widehat{A}}^{c} \tilde{a}}^{c},}
\end{aligned}
$$

for all $\tilde{a} \in \Gamma(\widehat{A})$.

Proof: The correspondence $X_{W} \mapsto \Delta_{W, A}$ between infinitesimal automorphisms and IM derivations is one-to-one by definition. Now, begin with $X_{W}$ and consider the associated data $\left(\Delta_{\widehat{A}}, \Delta_{E}, \Delta_{C}\right)$. The discussion immediately before the statement shows that $\Delta_{\widehat{A}}$ is a Lie algebroid derivation. Property (2.29) follows from the fact that all terms agree with the projection $X$ of $X_{W}$ on $M$. For property (2.30), it is enough to consider $\Phi$ of the form $\Phi=\varphi \otimes \chi$ (or equivalently $\Phi=\varphi \hat{\chi}$ ), where $\varphi \in \Gamma\left(E^{*}\right)$ and $\chi \in \Gamma(C)$. From the Leibniz rule for $\Delta_{W, E}$ it follows immediately that

$$
\Delta_{\widehat{A}} \Phi=\left(\Delta_{E}^{*} \varphi\right) \otimes \chi+\varphi \otimes \Delta_{C} \chi \in \Gamma(\operatorname{Hom}(E, C)) .
$$

Hence $\Delta_{\widehat{A}}$ restricts to a derivation of $\operatorname{Hom}(E, C)$. Additionally, from 2.29 we get also

$$
\left\langle\Delta_{\widehat{A}} \Phi, e\right\rangle=\left\langle\Delta_{E}^{*} \varphi, e\right\rangle \widehat{\chi}+\langle\varphi, e\rangle \widehat{\Delta_{C} \chi}=-\left\langle\varphi, \Delta_{E} e\right\rangle \widehat{\chi}+\Delta_{C}(\langle\varphi, e\rangle \widehat{\chi})=\left\langle\Delta_{C} \circ \Phi-\Phi \circ \Delta_{E}, e\right\rangle,
$$


for all $e \in \Gamma(E)$, i.e. 2.30 holds. For 2.31), fix $\chi \in \Gamma(C)$ and compute

$$
\left(\partial \Delta_{C} \chi\right)^{\uparrow}=\tilde{\rho}\left(\Delta_{W, E} \widehat{\chi}\right)=\left[\sigma\left(\Delta_{W, E}\right), \tilde{\rho}(\widehat{\chi})\right]=\left[X_{\Delta_{E}},(\partial \chi)^{\uparrow}\right]=\left(\Delta_{E} \partial \chi\right)^{\uparrow},
$$

where we used the fact that $\Delta_{W, E}$ is a Lie algebroid derivation of $W$ whose symbol is the linear vector field on $E$ corresponding to $\Delta_{E}$. For the same reason, for all $a \in \Gamma(\widehat{A})$ and $e \in \Gamma(E)$

$$
\left(\psi_{\Delta_{\widehat{A}} \tilde{a}}^{s} e\right)^{\uparrow}=\left[\tilde{\rho}\left(\Delta_{W, E} \tilde{a}\right), e^{\uparrow}\right]=\left[\left[\sigma\left(\Delta_{W, E}\right), \tilde{\rho}(\tilde{a})\right], e^{\uparrow}\right]=\left(\left[\Delta_{E}, \psi_{\tilde{a}}^{s}\right] e\right)^{\uparrow},
$$

which proves 2.32 and for all $\chi \in \Gamma(C)$,

$$
\widehat{\psi_{\Delta_{\widehat{A}}^{c} \tilde{a}} \chi}=\left[\Delta_{W, E} \tilde{a}, \hat{\chi}\right]=\Delta_{W, E}[\tilde{a}, \widehat{\chi}]-\left[\tilde{a}, \Delta_{W, E} \hat{\chi}\right]=\left[\widehat{\Delta_{C}, \psi_{\tilde{a}}^{c}}\right] \chi,
$$

which proves 2.33). Conversely, let $\left(\Delta_{\widehat{A}}, \Delta_{E}, \Delta_{C}\right)$ be as in the statement. From (2.29), and (2.30), $\left(\Delta_{\widehat{A}}, \Delta_{E}, \Delta_{C}\right)$ extends uniquely to an infinitesimal automorphism $X_{W}$ of the double vector bundle $(W \Rightarrow E ; A \Rightarrow M)$. From 2.31, 2.32), 2.33) and the fact that $\Delta_{\widehat{A}}$ is a Lie algebroid derivation, $X_{W}$ is an infinitesimal automorphism of the VB-algebroid structure as well. The details can be easily checked in local coordinates.

Let $\left(\Delta_{\widehat{A}}, \Delta_{E}, \Delta_{C}\right)$ be a triple as in the above theorem. Then, derivations $\Delta_{E}, \Delta_{C}$ agree with those in Remark 2.14. Notice that it follows from 2.30) that both pairs $\left(\Delta_{\widehat{A}}, \Delta_{E}\right)$ and $\left(\Delta_{\widehat{A}}, \Delta_{C}\right)$ determine the whole triple. Additionally $\Delta_{\widehat{A}}$ and derivation $\Delta_{A}$ from Remark 2.14 are $\pi$-related.

Remark 2.25 Equations (2.29) and (2.30) express compatibility with the double vector bundle structure, while Equations (2.31), 2.32), 2.33) express (the residual) compatibility with the algebroid structure.

From Proposition 3.16, Remark 2.22 and Theorem 2.24 the theorem stated below easily follows.

Theorem 2.26 Let $(\mathcal{W} \rightrightarrows E ; G \rightrightarrows M)$ be a VB-groupoid with VB-algebroid $(W \Rightarrow E ; A \Rightarrow M)$. Every infinitesimal automorphism $X_{\mathcal{W}}$ of $\mathcal{W}$ determines a triple $\left(\Delta_{\widehat{A}}, \Delta_{E}, \Delta_{C}\right)$ as in Theorem 2.24, via the following formulas

$$
\overrightarrow{\Delta_{\hat{A}} \tilde{\tilde{a}}}=\left[X_{\mathcal{W}}, \overrightarrow{\tilde{a}}\right], \quad X_{\Delta_{E}}=\tilde{s}_{*} X_{\mathcal{W}}=\tilde{t}_{*} X_{\mathcal{W}} \quad \text { and } \quad X_{\Delta_{C}}=\left.X_{\mathcal{W}}\right|_{C},
$$

or, equivalently,

$$
\vec{\Delta}_{\Delta_{\hat{A}} \tilde{a}}=\left[\Delta_{\mathcal{W}}, \vec{\Delta}_{\tilde{a}}\right], \quad \Delta_{E}=\tilde{s}_{*} \Delta_{\mathcal{W}}=\tilde{t}_{*} \Delta_{\mathcal{W}}, \quad \text { and } \quad \Delta_{C}=\left.\Delta_{\mathcal{W}}\right|_{C}
$$

for all $\tilde{a} \in \Gamma(\widehat{A})$. If $\mathcal{W} \rightrightarrows E$ is source simply connected, this correspondence is one-to-one.

Consider $X_{\mathcal{W}}$ to be an infinitesimal automorphism of the VB-groupoid ( $\mathcal{W} \rightrightarrows E ; G \rightrightarrows M$ ) and $X_{W}$ the infinitesimal automorphism of $(W \Rightarrow E ; A \Rightarrow M)=\mathrm{Lie}(\mathcal{W} \rightrightarrows E ; G \rightrightarrows M)$ associated to $X_{\mathcal{W}}$ by the Lie functor. Finally, let $\left(\Delta_{\widehat{A}}, \Delta_{E}, \Delta_{C}\right)$ be the algebraic data corresponding to $X_{W}$ via Theorem 2.24. The above theorem then states that derivation $\Delta_{E}$ (resp. $\Delta_{C}$ ) agrees with that of Remark 2.6.

\subsection{The linear deformation complex of a VB-groupoid/algebroid}

Multiplicative vector fields on a Lie group $G$ can be seen as 1-cocycles in the Lie group cohomology complex of $G$ with coefficients in the adjoint representation. More generally, multiplicative vector fields on a Lie groupoid $G \rightrightarrows M$ are 1-cocycles in the deformation complex of $G[14$. At the infinitesimal level, IM vector fields on a Lie algebroid $A \Rightarrow M$ are 1-cocycles in the deformation complex of $A[15]$. Additionally, the deformation complex of a Lie groupoid and the deformation 
complex of the associated Lie algebroid are intertwined by a Van Est map [14, which is yet another manifestation of the Lie functor. In this section we show that there is a natural cochain complex $C_{\text {def,lin }}^{\bullet}(\mathcal{W})\left(\right.$ resp. $C_{\text {def,lin }}^{\bullet}(W)$ ) attached to every VB-groupoid $(\mathcal{W} \rightrightarrows E ; G \rightrightarrows M)$ (resp. VB-algebroid $(W \Rightarrow E ; A \Rightarrow M))$ in such a way that multiplicative (resp. IM) derivations are the same as 1-cocycles, while internal derivations are equivalent to 1-coboundaries. Specifically, $C_{\text {def,lin }}^{\bullet}(\mathcal{W})\left(\right.$ resp. $\left.C_{\text {def,lin }}^{\bullet}(W)\right)$ is a subcomplex of the deformation complex of the Lie groupoid $\mathcal{W} \rightrightarrows E$ (resp. Lie algebroid $W \rightarrow E$ ) consisting of cochains that are linear in a suitable sense (see below). Additionally we show that the Van Est map restricts to a cochain map intertwining $C_{\text {def,lin }}^{\bullet}(\mathcal{W})$ and $C_{\text {def,lin }}^{\bullet}(W)$. Notice that this is similar to what Cabrera and Drummond do in 9. There is one main difference though. While they work with the Lie groupoid/algebroid cohomology of the total space of a VB-groupoid/algebroid, we work with the deformation complex. In this respect we recall that the deformation complex $C_{\mathrm{def}}^{\bullet}(\mathcal{W})$ of a Lie groupoid $\mathcal{W} \rightrightarrows E$ with Lie algebroid $W \Rightarrow E$, can be seen as a subcomplex in the Lie groupoid complex $C^{\bullet}\left(T^{*} \mathcal{W}\right.$ ) of the cotangent groupoid $T^{*} \mathcal{W} \rightrightarrows W_{E}^{*}\left[14\right.$ (where $W_{E}^{*} \rightarrow E$ is the dual of the vector bundle $W \rightarrow E)$. Specifically, $C_{\text {def }}^{\bullet}(\mathcal{W})$ is the subcomplex consisting of cochains that are linear with respect to the vector bundle structure $T^{*} \mathcal{W} \rightarrow \mathcal{W}$, plus an additional projectability condition. If $\mathcal{W}$ is the total space of a VB-groupoid, then $T^{*} \mathcal{W}$ is actually a triple structure: a double vector bundle in the category of groupoids. This means that the results in this sections are analogous to those in [9] but for groupoids equipped with two (not just one) compatible vector bundle structures.

Let us recall how is the deformation complex defined. Let $G \rightrightarrows M$ be a Lie groupoid with Lie algebroid $A$. Following [14], we denote by $\bar{m}$ the division map in $G$, i.e.

$$
\bar{m}(g, h)=g h^{-1}
$$

for all $g, h \in G$ such that $s(g)=s(h)$. For any $k \geq 0$, we also denote by $G^{(k)}$ the space of $k$-tuples of composable arrows. In particular, $G^{(0)}=M$ and $G^{(1)}=G$. The deformation complex of $G$ [14 is the pair $\left(C_{\mathrm{def}}^{\bullet}(G), \delta\right)$, where, for $k>0, C_{\mathrm{def}}^{k}(G)$ is the space of smooth maps

$$
c: G^{(k)} \longrightarrow T G, \quad\left(g_{1}, \ldots, g_{k}\right) \longmapsto c\left(g_{1}, \ldots, g_{k}\right) \in T_{g_{1}} G,
$$

which are source-projectable, i.e. there exists a (necessarily unique) smooth map $\sigma_{c}: G^{(k-1)} \longrightarrow$ $T M, \quad\left(g_{1}, \ldots, g_{k-1}\right) \longmapsto \sigma_{c}\left(g_{1}, \ldots, g_{k-1}\right) \in T_{t\left(g_{1}\right)} M$, called the source projection of $c$, such that $(\mathrm{d} s \circ c)\left(g_{1}, g_{2}, \ldots, g_{k}\right)=\sigma_{c}\left(g_{2}, \ldots, g_{k}\right)$. So 1-cochains are exactly source-projectable vector fields on $G$. The differential $\delta c$ of $c \in C_{\text {def }}^{k}(G)$ is defined by

$$
\begin{aligned}
(\delta c)\left(g_{1}, \ldots, g_{k+1}\right)= & -\mathrm{d} \bar{m}\left(c\left(g_{1} g_{2}, g_{3}, \ldots, g_{k+1}\right), c\left(g_{2}, g_{3}, \ldots, g_{k+1}\right)\right) \\
& +\sum_{i=2}^{k}(-)^{i} c\left(g_{1}, \ldots, g_{i} g_{i+1}, \ldots, g_{k+1}\right)+(-)^{k+1} c\left(g_{1}, \ldots, g_{k}\right) .
\end{aligned}
$$

For $k=0, C_{\mathrm{def}}^{0}(G):=\Gamma(A)$ and for $a \in \Gamma(A), \delta a:=\vec{a}+\overleftarrow{a}$. The terminology is motivated by the fact that $\left(C_{\text {def }}^{\bullet}(G), \delta\right)$ controls deformations of $G \rightrightarrows M[14$. It is easy to see that 1-cocycles in $\left(C_{\text {def }}^{\bullet}(G), \delta\right)$ are the same as multiplicative vector fields on $G$ (see [14, Proposition 4.3]). We also consider the normalized deformation complex of $G$ as the subcomplex $\hat{C}_{\text {def }}^{\bullet}(G) \subset C_{\text {def }}^{\bullet}(G)$ consisting of normalized cochains. For $k \geq 2$, a normalized $k$-cochain is a $k$-cochain $c$ such that for all $x \in M$

$$
c\left(1_{x}, g_{2}, \ldots, g_{k}\right)=\sigma_{c}\left(g_{2}, \ldots, g_{k}\right) \text { and } c\left(g_{1}, g_{2}, \ldots, 1_{x}, \ldots, g_{k}\right)=0 .
$$

In degree 1 , the only condition is that $c\left(1_{x}\right)=\left(\sigma_{c}\right)_{x}$, and in degree 0 there are no conditions.

Now, let $(\mathcal{W} \rightrightarrows E ; G \rightrightarrows M)$ be a VB-groupoid with VB-algebroid $(W \Rightarrow E ; A \Rightarrow M)$. Consider the subspace $C_{\text {def,lin }}^{\bullet}(\mathcal{W})$ of $C_{\text {def }}^{\bullet}(\mathcal{W})$ defined as follows. For $k>0, C_{\text {def,lin }}^{k}(\mathcal{W})$ consists of those cochains $\tilde{c} \in C_{\mathrm{def}}^{k}(\mathcal{W})$ which are linear, i.e. $\tilde{c}: \mathcal{W}^{(k)} \rightarrow T \mathcal{W}$ is a vector bundle morphism covering a 
(necessarily unique) smooth map $c: G^{(k)} \rightarrow T G$ :

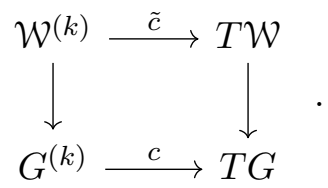

In particular $C_{\text {def,lin }}^{1}(\mathcal{W})$ consists of those source-projectable vector fields on the Lie groupoid $\mathcal{W}$ which are also infinitesimal automorphisms of the vector bundle $\mathcal{W} \rightarrow G$. For $k=0, C_{\text {def,lin }}^{0}(\mathcal{W}):=$ $\Gamma_{\ell}(W, E)=\Gamma(\widehat{A})$, where $\widehat{A} \Rightarrow M$ is the fat algebroid of $(W \Rightarrow E ; A \Rightarrow M)$.

\section{Lemma 2.27}

i.) $C_{\mathrm{def}, \mathrm{lin}}(\mathcal{W})$ is a subcomplex of $\left(C_{\mathrm{def}}^{\bullet}(\mathcal{W}), \delta\right)$.

ii.) If $\tilde{c}: \mathcal{W}^{(k)} \rightarrow T \mathcal{W}$ belongs to $C_{\mathrm{def}, \text { lin }}^{k}(\mathcal{W})$, then its projection $c: G^{(k)} \rightarrow T G$ belongs to $C_{\mathrm{def}}^{k}(G)$ and $\delta \tilde{c}$ projects to $\delta c$.

Proof: Let $\tilde{a} \in C_{\mathrm{def}, \text { lin }}^{0}(\mathcal{W})$ be a linear section of $W \rightarrow E$. Then $\delta \tilde{a}=\overrightarrow{\vec{a}}+\overleftarrow{\tilde{a}}$ is an infinitesimal automorphism of $(\mathcal{W} \rightrightarrows E ; G \rightrightarrows M)$ (Remark 2.9. In particular, it belongs to $C_{\mathrm{def}, \text { lin }}^{1}(\mathcal{W})$. Now, for $k>0$, let $\tilde{c}: \mathcal{W}^{(k)} \rightarrow T \mathcal{W}$ belong to $C_{\text {def,lin }}^{k}(\mathcal{W})$, and show that $\delta \tilde{c}$ belongs to $C_{\text {def,lin }}^{k+1}(\mathcal{W})$. This follows, after a straightforward computation, from the linearity of $\tilde{c}$ and the obvious fact that like the (other) structure maps, the division $\overline{\tilde{m}}=\mathcal{W}_{\tilde{s}} \times_{\tilde{s}} \mathcal{W} \rightarrow \mathcal{W}$ in a VB-groupoid $(\mathcal{W} \rightrightarrows E ; G \rightrightarrows M$ ) is a vector bundle morphism (covering the division $\bar{m}: G_{s} \times_{s} G \rightarrow G$ in $G$ ). The second part of the statement immediately follows from the axioms of VB-groupoids and the, easy to check, commutativity of the following diagram:

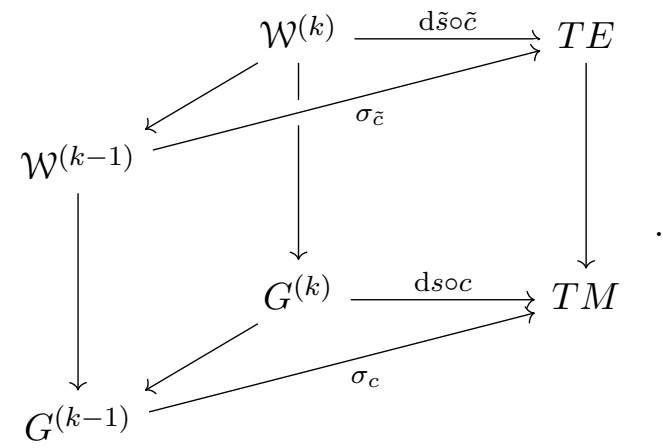

Here the projection $\mathcal{W}^{(k)} \rightarrow \mathcal{W}^{(k-1)}$ consists in dropping the first entry (and similarly for the projection $G^{(k)} \rightarrow G^{(k-1)}$ ) and $\sigma_{c}=\mathrm{d} q \circ \sigma_{\tilde{c}} \circ 0_{\mathcal{W}^{(k-1)}}$, where $0_{\mathcal{W}^{(k-1)}}: G^{(k-1)} \rightarrow \mathcal{W}^{(k-1)}$ is the zero section.

\section{Proposition 2.28}

i.) Infinitesimal automorphisms of $(\mathcal{W} \rightrightarrows E ; G \rightrightarrows M)$ are 1 -cocycles in $\left(C_{\mathrm{def}, \operatorname{lin}}^{\bullet}(\mathcal{W}), \delta\right)$.

ii.) Internal derivations of $(\mathcal{W} \rightrightarrows E ; G \rightrightarrows M)$ are (equivalent to) 1 -coboundaries in $\left(C_{\operatorname{def}, \operatorname{lin}}(\mathcal{W}), \delta\right)$.

Proof: It immediately follows from Remark 2.9 and [14, Proposition 4.3].

We now pass to the infinitesimal picture. Recall from 15 that the deformation complex of a Lie algebroid $A \Rightarrow M$ is the pair $\left(C_{\mathrm{def}}^{\bullet}(A), \delta\right)$, where, for $k>0, C_{\mathrm{def}}^{k}(A)$ consists of (skew-symmetric) multiderivations

$$
c: \Gamma(A) \times \cdots \times \Gamma(A) \longrightarrow \Gamma(A)
$$


with $k$-entries, and multilinear symbol, i.e. $c$ is a skew-symmetric $\mathbb{R}$-multilinear map, and there exists a (necessarily unique) vector bundle morphism $\sigma_{c}: \wedge^{k-1} A \rightarrow T M$, called the symbol of $c$, such that

$$
c\left(a_{1}, \ldots, a_{k-1}, f a_{k}\right)=f c\left(a_{1}, \ldots, a_{k-1}, a_{k}\right)+\sigma_{c}\left(a_{1}, \ldots, a_{k-1}\right)(f) a_{k},
$$

for all $a_{1}, \ldots, a_{k} \in \Gamma(A)$ and $f \in C^{\infty}(M)$. In particular 1-cochains are just derivations of the vector bundle $A \rightarrow M$. The differential $\delta c$ of $c \in C_{\mathrm{def}}^{k}(A)$ is defined by

$$
\begin{aligned}
(\delta c)\left(a_{1}, \ldots, a_{k+1}\right)= & \sum_{i=1}^{k+1}(-)^{i+1}\left[a_{i}, c\left(a_{1}, \ldots, \widehat{a}_{i}, \ldots, a_{k+1}\right)\right] \\
& +\sum_{i<j}(-)^{i+j} c\left(\left[a_{i}, a_{j}\right], a_{1}, \ldots, \widehat{a}_{i}, \ldots, \widehat{a}_{j}, \ldots, a_{k+1}\right),
\end{aligned}
$$

for all $a_{1}, \ldots, a_{k+1} \in \Gamma(A)$. A straightforward computation then shows that

$$
\begin{aligned}
\sigma_{\delta c}\left(a_{1}, \ldots, a_{k}\right)= & \sum_{i=1}^{k}(-)^{i+1}\left[\rho\left(a_{i}\right), \sigma_{c}\left(a_{1}, \ldots, \widehat{a}_{i}, \ldots, a_{k}\right)\right] \\
& +\sum_{i<j}(-)^{i+j} \sigma_{c}\left(\left[a_{i}, a_{j}\right], a_{1}, \ldots, \widehat{a}_{i}, \ldots, \widehat{a}_{j}, \ldots, a_{k}\right)-(-)^{k} \rho\left(c\left(a_{1}, \ldots, a_{k}\right)\right) .
\end{aligned}
$$

for all $a_{1}, \ldots, a_{k} \in \Gamma(A)$. For $k=0, C_{\mathrm{def}}^{0}(A):=\Gamma(A)$ and for $a \in \Gamma(A), \delta a:=[a,-]$. The terminology is motivated by the fact that $\left(C_{\text {def }}^{\bullet}(A), \delta\right)$ controls deformations of $A$ [15] (see also [39, Section 1.1]). We remark, however, that the complex $\left(C_{\text {def }}^{\bullet}(A), \delta\right)$ first appeared in [25] under a different name, and for different purposes (see Theorem 6 loc. cit.).

It is immediate that 1-cocycles in $\left(C_{\text {def }}^{\bullet}(A), \delta\right)$ are the same as Lie algebroid derivations of $A$ (cf. [15, Section 3.1]). Hence they are in one-to-one correspondence with IM vector fields on $A$.

Remark 2.29 The graded space $C_{\text {def }}^{\bullet}(A)$ is not just a cochain complex. Actually, up to a shift, it becomes a dg-Lie algebra when additionally equipped with the Schouten-Jacobi bracket $\llbracket-,-\rrbracket$ (see [41,42]), originally named the Gerstenhaber bracket in [15. Differential $\delta$ is then $\llbracket b,-\rrbracket$, where $b=[-,-]$ is the Lie bracket on $\Gamma(A)$.

When $A$ is the Lie algebroid of a Lie groupoid $G \rightrightarrows M$, then the deformation complex of $A$ and the normalized deformation complex of $G$ are intertwined by a Van Est map [14], i.e. the cochain map

$$
\mathcal{V}:\left(\widehat{C}_{\mathrm{def}}^{\bullet}(G), \delta\right) \longrightarrow\left(C_{\mathrm{def}}^{\bullet}(A), \delta\right)
$$

defined by

$$
(\mathcal{V} c)\left(a_{1}, \ldots, a_{k}\right):=\sum_{\tau \in S_{k}}(-)^{\tau}\left(R_{a_{\tau(1)}} \circ \cdots \circ R_{a_{\tau(k)}}\right)(c),
$$

for all $c \in \widehat{C}_{\mathrm{def}}^{k}(G)$ and $a_{1}, \ldots, a_{k} \in \Gamma(A)$. Here, for any $a \in \Gamma(A)$,

$$
R_{a}: \widehat{C}_{\mathrm{def}}^{\bullet+1}(G) \rightarrow \widehat{C}_{\mathrm{def}}^{\bullet}(G)
$$

is the map defined as follows. For $c \in \widehat{C}_{\mathrm{def}}^{1}(G), R_{a} c:=\left.[c, \vec{a}]\right|_{M}$ and for $c \in \widehat{C}_{\mathrm{def}}^{k+1}(G), k>0$,

$$
\left(R_{a} c\right)\left(g_{1}, \ldots, g_{k}\right):=\left.(-)^{k} \frac{d}{d \varepsilon}\right|_{\varepsilon=0} c\left(g_{1}, \ldots, g_{k}, \phi_{\varepsilon}^{a}\left(s\left(g_{k}\right)\right)^{-1}\right),
$$

where $\left\{\phi_{\varepsilon}^{a}\right\}$ is the flow of $\vec{a}$. Now, let $(W \Rightarrow E ; A \Rightarrow M)$ be a VB-algebroid. Consider the subspace $C_{\text {def,lin }}^{\bullet}(W)$ of $C_{\text {def }}^{\bullet}(W)$ defined as follows. For $k>0, C_{\text {def,lin }}^{k}(W)$ consists of linear cochains, i.e. those multiderivations $\tilde{c}: \Gamma(W, E) \times \cdots \times \Gamma(W, E) \rightarrow \Gamma(W, E)$ such that

$$
\tilde{c}\left(\tilde{a}_{1}, \ldots, \tilde{a}_{k}\right) \text { is a linear section, }
$$




$$
\begin{gathered}
\tilde{c}\left(\tilde{a}_{1}, \ldots, \tilde{a}_{k-1}, \widehat{\chi}_{1}\right) \text { is a core section, } \\
\tilde{c}\left(\tilde{a}_{1}, \ldots, \tilde{a}_{k-i}, \widehat{\chi}_{1}, \ldots, \widehat{\chi}_{i}\right)=0,
\end{gathered}
$$

and, additionally

$$
\begin{gathered}
\sigma_{\tilde{c}}\left(\tilde{a}_{1}, \ldots, \tilde{a}_{k-1}\right) \text { is a linear vector field, } \\
\sigma_{\tilde{c}}\left(\tilde{a}_{1}, \ldots, \tilde{a}_{k-2}, \widehat{\chi}_{1}\right) \text { is the vertical lift of a section of } E, \\
\sigma_{\tilde{c}}\left(\tilde{a}_{1}, \ldots, \tilde{a}_{k-i-1}, \widehat{\chi}_{1}, \ldots, \widehat{\chi}_{i}\right)=0,
\end{gathered}
$$

for all linear sections $\tilde{a}_{1}, \ldots, \tilde{a}_{k}$, all core sections $\widehat{\chi}_{1}, \ldots, \widehat{\chi}_{i}$ of $W \rightarrow E$, and all $i \geq 2$. For $k=0$, we define $C_{\text {def,lin }}^{0}(W):=\Gamma_{\ell}(W, E)=\Gamma(\widehat{A})$.

Lemma 2.30 $C_{\mathrm{def}, l i n}^{\bullet}(W)$ is a subcomplex, and a Lie subalgebra, in $C_{\mathrm{def}}^{\bullet}(W)$.

Proof: It immediately follows from the explicit formula for $\delta$, Definition 1.5 and the explicit formula for the Schouten-Jacobi bracket (see 15, Proposition 1]).

Linear 0-cochains in $C_{\text {def }}^{\bullet}(W)$ are already characterized by Lemma 2.17. For higher degree cochains the situation is illustrated by the following

Lemma 2.31 For any $k \geq 0$, a cochain $\tilde{c} \in C_{\mathrm{def}}^{k}(W)$ is linear if and only if $\llbracket \Delta_{\mathcal{E}}, \tilde{c} \rrbracket=0$, where $\llbracket-,-\rrbracket$ is the Schouten-Jacobi bracket (see Remark 2.29).

ProOF: First recall that a multiderivation is completely determined by its own action and its symbol action on generators. From the explicit formula for the Schouten-Jacobi bracket 15, Proposition 1] it follows that

$$
\llbracket \Delta_{\mathcal{E}}, \tilde{c} \rrbracket\left(w_{1}, \ldots, w_{k}\right)=\Delta_{\mathcal{E}}\left(\tilde{c}\left(w_{1}, \ldots, w_{k}\right)\right)-\sum_{i=1}^{k} \tilde{c}\left(w_{1}, \ldots, \Delta_{\mathcal{\varepsilon}} w_{i}, \ldots, w_{k}\right),
$$

and

$$
\sigma_{\llbracket \Delta_{\varepsilon}, \tilde{c} \rrbracket}\left(w_{1}, \ldots, w_{k-1}\right)=\left[\sigma\left(\Delta_{\mathcal{\varepsilon}}\right), \sigma_{\tilde{c}}\left(w_{1}, \ldots, w_{k-1}\right)\right]-\sum_{i=1}^{k-1} \sigma_{\tilde{c}}\left(w_{1}, \ldots, \Delta_{\mathcal{\varepsilon}} w_{i}, \ldots, w_{k-1}\right),
$$

for all $w_{1}, \ldots, w_{k} \in \Gamma(W, E)$. Using 2.62 and Lemma 2.17 we immediately see that if $\tilde{c}$ is linear, then $\llbracket \Delta_{\varepsilon}, \tilde{c} \rrbracket$ and its symbol vanish on linear and core sections. Hence $\llbracket \Delta_{\varepsilon}, \tilde{c} \rrbracket=0$. Conversely, suppose that $\llbracket \Delta_{\varepsilon}, \tilde{c} \rrbracket=0$. Then (2.56), (2.57), and (2.58) follow from (2.62) and Lemma 2.17. Similarly, (2.59), (2.60), and (2.61) follow from (2.63), the fact that $\sigma\left(\Delta_{\varepsilon}\right)$ is the Euler vector field on $E$ (cf. Remark 2.1) and that there are no non-trivial vector fields $X$ on $E$ such that $\left[\sigma\left(\Delta_{\varepsilon}\right), X\right]=-i X$ for some integer $i>1$ (cf. Lemma 2.17).

\section{Corollary 2.32}

i.) Infinitesimal automorphisms of $(W \Rightarrow E ; A \Rightarrow M)$ are equivalent to 1-cocycles in $\left(C_{\mathrm{def}, \operatorname{lin}}(W), \delta\right)$.

ii.) Internal derivations of $(W \Rightarrow E ; A \Rightarrow M)$ are equivalent to 1-coboundaries in $\left(C_{\mathrm{def}, \mathrm{lin}}(W), \delta\right)$.

Proof: It immediately follows from Remark 2.18, Lemma 2.31 and 15, Section 3.1].

Now we discuss the analogue of Theorem 2.24 for cochains in $C_{\mathrm{def}, \operatorname{lin}}^{k}(W)$. Namely, let $\tilde{c} \in C_{\mathrm{def}, \mathrm{lin}}^{k}$, $k>1$. By definition, $\tilde{c}$ preserves linear sections, hence it determines by restriction a $k$-cochain $c_{\widehat{A}}$ in the fat algebroid $\widehat{A}$. Additionally, $\sigma_{\tilde{c}}$ maps linear sections to linear vector fields and we set

$$
c_{E}\left(\tilde{a}_{1}, \ldots, \tilde{a}_{k-1}\right):=\Delta_{\sigma_{\tilde{c}}\left(\tilde{a}_{1}, \ldots, \tilde{a}_{k-1}\right)},
$$


for all $\tilde{a}_{1}, \ldots, \tilde{a}_{k-1} \in \Gamma(\widehat{A})$ (recall that $\Delta_{X}$ denotes the derivation corresponding to a linear vector field $X$ ). Moreover, $\tilde{c}$ maps $k-1$ linear sections and a core sections to core sections and we set

$$
c_{C}\left(\tilde{a}_{1},{\widehat{,}, \tilde{a}_{k-1}}\right)(\chi):=\tilde{c}\left(\tilde{a}_{1}, \ldots, \tilde{a}_{k-1}, \widehat{\chi}\right),
$$

for all $\tilde{a}_{1}, \ldots, \tilde{a}_{k-1} \in \Gamma(\widehat{A})$ and $\chi \in \Gamma(C)$ (recall that $\widehat{\chi}$ is the core section of $W \rightarrow E$ corresponding to $\chi$ ). Finally, we define $\mathrm{d}_{\widehat{A}}$ by

$$
\mathrm{d}_{\widehat{A}}\left(\tilde{a}_{1}, \ldots, \tilde{a}_{k-2}\right)(\chi)^{\uparrow}:=\sigma_{\tilde{c}}\left(\tilde{a}_{1}, \ldots, \tilde{a}_{k-2}, \widehat{\chi}\right),
$$

(recall that $e^{\uparrow}$ denotes the vertical lift of a section $e \in \Gamma(E)$ ). For $k=1$, a cochain $\tilde{c} \in C_{\text {def,lin }}^{1}(W)$ is a derivation of $W \rightarrow E$ with the additional property of preserving linear sections. It is then obvious that the corresponding linear vector field $X_{\tilde{c}}$ on $W$ is an infinitesimal automorphism of the double vector bundle $(W \rightarrow E ; A \rightarrow M)$. In particular, we can associate to $X_{\tilde{c}}$ a triple $\left(\Delta_{\widehat{A}}, \Delta_{E}, \Delta_{C}\right)$ exactly as we did in Theorem 2.24, except for the fact that as $X_{\tilde{c}}$ is not an infinitesimal automorphism of the VB-algebroid in general, $\left(\Delta_{\widehat{A}}, \Delta_{E}, \Delta_{C}\right)$ do only satisfy Equations $(2.29)$ and $(2.30)$, and do not satisfy (2.31), 2.32) and 2.33) in general (see also Remark 2.25). In this section we will denote by $\left(c_{\widehat{A}}, c_{E}, c_{C}\right)$ the triple $\left(\Delta_{\widehat{A}}, \Delta_{E}, \Delta_{C}\right)$ to stress that it comes from a (non-necessarily closed) 1-cochain in $C_{\mathrm{def}, \operatorname{lin}}^{\bullet}(W)$.

\section{Theorem 2.33}

i.) For $k>1$, the assignment $\tilde{c} \mapsto\left(c_{\widehat{A}}, c_{E}, c_{C}, \mathrm{~d}_{\widehat{A}}\right)$ establishes a one-to-one correspondence between cochains in $C_{\mathrm{def}, \mathrm{lin}}^{k}(W)$ and 4-tuples consisting of

- a $k$-cochain $c_{\widehat{A}}$ in the deformation complex of the fat algebroid $\widehat{A}$,

- a vector bundle morphism $c_{E}: \wedge^{k-1} \widehat{A} \rightarrow \mathrm{D} E$,

- a vector bundle morphism $c_{C}: \wedge^{k-1} \widehat{A} \rightarrow \mathrm{D} C$ and

- a vector bundle morphism $\mathrm{d}_{\widehat{A}}: \wedge^{k-2} \widehat{A} \rightarrow \operatorname{Hom}(C, E)$, such that

$$
\sigma \circ c_{E}=\sigma \circ c_{C}=\sigma_{c_{\widehat{A}}}
$$

where $\sigma: \mathrm{DE} \rightarrow T M$ (resp. $\sigma: \mathrm{DC} \rightarrow T M$ ) is the symbol map and additionally

$$
\begin{aligned}
& c_{\widehat{A}}\left(\tilde{a}_{1}, \ldots, \tilde{a}_{k-1}, \Phi\right)=c_{C}\left(\tilde{a}_{1}, \ldots, \tilde{a}_{k-1}\right) \circ \Phi-\Phi \circ c_{E}\left(\tilde{a}_{1}, \ldots, \tilde{a}_{k-1}\right), \\
& c_{E}\left(\tilde{a}_{1}, \ldots, \tilde{a}_{k-2}, \Phi\right)=-\mathrm{d}_{\widehat{A}}\left(\tilde{a}_{1}, \ldots, \tilde{a}_{k-2}\right) \circ \Phi, \\
& c_{C}\left(\tilde{a}_{1}, \ldots, \tilde{a}_{k-2}, \Phi\right)=-\Phi \circ \mathrm{d}_{\widehat{A}}\left(\tilde{a}_{1}, \ldots, \tilde{a}_{k-2}\right), \\
& \mathrm{d}_{\widehat{A}}\left(\tilde{a}_{1}, \ldots, \tilde{a}_{k-3}, \Phi\right)=0
\end{aligned}
$$

for all $\tilde{a}_{1}, \ldots, \tilde{a}_{k-1} \in \Gamma(\widehat{A})$ and all $\Phi \in \Gamma(\operatorname{Hom}(E, C))$.

If $\tilde{c}$ corresponds to $\left(c_{\widehat{A}}, c_{E}, c_{C}, \mathrm{~d}_{\widehat{A}}\right)$, then $\delta \tilde{c}$ corresponds to $\left(\delta c_{\widehat{A}}, c_{E}^{\prime}, c_{C}^{\prime}, \mathrm{d}_{\widehat{A}}^{\prime}\right)$ where

$$
\begin{aligned}
c_{E}^{\prime}\left(\tilde{a}_{1}, \ldots, \tilde{a}_{k}\right)= & \sum_{i=1}^{k}(-)^{i+1}\left[\psi_{\tilde{a}_{i}}^{s}, c_{E}\left(\tilde{a}_{1}, \ldots, \widehat{\tilde{a}_{i}}, \ldots, \tilde{a}_{k}\right)\right] \\
& +\sum_{i<j}(-)^{i+j} c_{E}\left(\left[\tilde{a}_{i}, \tilde{a}_{j}\right], \tilde{a}_{1}, \ldots, \widehat{\hat{a}_{i}}, \ldots, \widehat{\widehat{a}_{j}}, \ldots, \tilde{a}_{k}\right)-(-)^{k} \psi_{c_{\widehat{A}}\left(\tilde{a}_{1}, \ldots, \tilde{a}_{k}\right)},
\end{aligned}
$$


and like-wise for $c_{C}^{\prime}$ and

$$
\begin{aligned}
& \mathrm{d}_{\widehat{A}}^{\prime}\left(\tilde{a}_{1}, \ldots, \tilde{a}_{k-1}\right) \\
= & \sum_{i=1}^{k-1}(-)^{i+1}\left(\psi_{\tilde{a}_{i}}^{s} \circ \mathrm{d}_{\widehat{A}}\left(\tilde{a}_{1}, \ldots, \widehat{\tilde{a}}_{i}, \ldots, \tilde{a}_{k-1}\right)-\mathrm{d}_{\widehat{A}}\left(\tilde{a}_{1}, \ldots, \widehat{\tilde{a}}_{i}, \ldots, \tilde{a}_{k-1}\right) \circ \psi_{\tilde{a}_{i}}^{c}\right) \\
& +\sum_{i<j}(-)^{i+j} \mathrm{~d}_{\widehat{A}}\left(\left[\tilde{a}_{i}, \tilde{a}_{j}\right], \tilde{a}_{1}, \ldots, \widehat{\tilde{a}}_{i}, \ldots, \widehat{\tilde{a}}_{j}, \ldots, \tilde{a}_{k-1}\right) \\
& +(-)^{k}\left(c_{E}\left(\tilde{a}_{1}, \ldots, \tilde{a}_{k-1}\right) \circ \partial-\partial \circ c_{C}\left(\tilde{a}_{1}, \ldots, \tilde{a}_{k-1}\right)\right),
\end{aligned}
$$

for all $\tilde{a}_{1}, \ldots, \tilde{a}_{k} \in \Gamma(\widehat{A})$.

ii.) For $k=1$, the assignment $\tilde{c} \mapsto\left(c_{\widehat{A}}, c_{E}, c_{C}\right)$ establishes a one-to-one correspondence between 1-cochains in $C_{\mathrm{def}, \mathrm{in}}(W)$ and triples $\left(c_{\widehat{A}}, c_{E}, c_{C}\right)$, where $c_{\widehat{A}}, c_{E}, c_{C}$ are derivations of the vector bundles $\widehat{A}, E, C$ respectively, such that

$$
\sigma\left(c_{E}\right)=\sigma\left(c_{C}\right)=\sigma\left(c_{\widehat{A}}\right)
$$

and

$$
c_{\widehat{A}}(\Phi)=c_{C} \circ \Phi-\Phi \circ c_{E}
$$

for all $\Phi \in \Gamma(\operatorname{Hom}(E, C))$. If $\tilde{c}$ corresponds to $\left(c_{\widehat{A}}, c_{E}, c_{C}\right)$, then $\delta \tilde{c} \in C_{\mathrm{def}, \text { lin }}^{2}(W)$ corresponds to the 4-tuple $\left(\delta c_{\widehat{A}}, c_{E}^{\prime}, c_{C}^{\prime}, \mathrm{d}_{\widehat{A}}^{\prime}\right)$, where

$$
\mathrm{d}_{\widehat{A}}^{\prime}=\partial \circ c_{C}-c_{E} \circ \partial
$$

and for all $\tilde{a} \in \Gamma(\widehat{A})$,

$$
c_{E}^{\prime}(\tilde{a})=\left[\psi_{\tilde{a}}^{s}, c_{E}\right]+\psi_{c_{\widehat{A}}(\tilde{a})}^{s}, \quad c_{C}^{\prime}(\tilde{a})=\left[\psi_{\tilde{a}}^{c}, c_{C}\right]+\psi_{c_{\widehat{A}}(\tilde{a})}^{c} .
$$

iii.) For $k=0$, the 0 -cochains $\tilde{c}$ in $C_{\mathrm{def}, \operatorname{lin}}^{\bullet}(W)$ are the same as sections of the fat algebroid $\widehat{A} \rightarrow M$. If $c \in C_{\mathrm{def}, \operatorname{lin}}^{0}(W)$ is given by $\tilde{a} \in \Gamma(\widehat{A})$, then $\delta c$ corresponds to the triple $\left(\delta \tilde{a}, \psi_{\tilde{a}}^{s}, \psi_{\tilde{a}}^{c}\right)$.

Proof: The second and the third claims are straightforward. Let us prove the first one. Let $\tilde{c} \in C_{\mathrm{def}, \text { lin }}^{k}(W)$. It is clear from the definition that $c_{\widehat{A}}$ is a multiderivation, both $c_{E}$ and $d_{\widehat{A}}$ are multi-linear and 2.67) holds. In order to prove the multi-linearity of $c_{C}$ (in its first $k-1$ arguments), let $f \in C^{\infty}(M)$ and compute

$$
\tilde{c}\left(\tilde{a}_{1}, \ldots \tilde{a}_{k-2}, f \tilde{a}_{k-1}, \widehat{\chi}\right)=f \tilde{c}\left(\tilde{a}_{1}, \ldots \tilde{a}_{k-2}, \tilde{a}_{k-1}, \widehat{\chi}\right)-\sigma_{\tilde{c}}\left(\tilde{a}_{1}, \ldots \tilde{a}_{k-2}, \hat{\chi}\right)(f) \tilde{a}_{k-1} .
$$

Since $\sigma_{\tilde{c}}\left(\tilde{a}_{1}, \ldots \tilde{a}_{k-2}, \widehat{\chi}\right)$ is a vertical vector field on $E$, the last summand vanishes and we get $C^{\infty}(M)$ linearity in $\tilde{a}_{k-1}$. Now, we check 2.68 -2.71). It is enough to consider $\Phi$ of the form $\Phi=\varphi \otimes \chi$, where $\varphi \in \Gamma\left(E^{*}\right)$ and $\chi \in \Gamma(C)$. Hence $\Phi$ corresponds to the linear section $\varphi \hat{\chi}$, where, as usual, we interpret $\varphi$ as a fiber-wise linear function on $E$, and 2.68 2.70 easily follow from either the Leibniz rule for $\tilde{c}$ or the multi-linearity of $\sigma_{\tilde{c}}$, while 2.71) follows from 2.61). As already recalled, a multiderivation $c$ of a vector bundle is completely determined by its action and the action of $\sigma_{c}$ on generators of the module of sections of the vector bundle. As linear and core sections generate $\Gamma(W, E)$ as a $C^{\infty}(E)$-module, then the data $\left(c_{\widehat{A}}, c_{E}, c_{C}, \mathrm{~d}_{\widehat{A}}\right)$ determine $\tilde{c} \in C_{\text {def,lin }}(W)$ completely, i.e. the correspondence $\tilde{c} \mapsto\left(c_{\widehat{A}}, c_{E}, c_{C}, \mathrm{~d}_{\widehat{A}}\right)$ is injective. To see that every 4-tuple $\left(c_{\widehat{A}}, c_{E}, c_{C}, \mathrm{~d}_{\widehat{A}}\right)$ as in the statement arises in this way, use local coordinates to show that $\tilde{c} \mapsto\left(c_{\widehat{A}}, c_{E}, c_{C}, \mathrm{~d}_{\widehat{A}}\right)$ is a $(\mathrm{n}$ injective) $C^{\infty}(M)$-linear map between modules of sections of two vector bundles over $M$ of the same rank. Finally, (2.72) and (2.73) follow from (2.50) and (2.51) by a straightforward computation. 
Let $\left(c_{\widehat{A}}, c_{E}, c_{C}, \mathrm{~d}_{\widehat{A}}\right)$ be a 4 -tuple as in the statement of the above theorem. Then condition (2.67) says that $c_{E}$ and $c_{C}$ are derivation valued skew-symmetric $k$-forms on $\widehat{A}$ in the sense of $[59$, Section 2.4]. Additionally, it follows from 2.68 that $c_{\widehat{A}}$ descends to a $k$-cochain in the deformation complex of $A$, i.e.

$$
c_{A}(\pi(-), \ldots, \pi(-))=\pi \circ c_{\widehat{A}},
$$

for some $c_{A} \in C_{\text {def }}^{k}(A)$, where $\pi: \widehat{A} \rightarrow A$ is the projection. Finally, from 2.71), $\mathrm{d}_{\widehat{A}}$ descends to a vector bundle morphism $\mathrm{d}_{A}: \wedge^{k-1} A \rightarrow \operatorname{Hom}(C, E)$, i.e.

$$
\mathrm{d}_{\widehat{A}}=\mathrm{d}_{A}(\pi(-), \ldots, \pi(-)) .
$$

We conclude this subsection showing that the deformation complex of a VB-groupoid and the deformation complex of its VB-algebroid are intertwined by a linear Van Est map.

Theorem 2.34 (Linear Van Est map) Let (W $\rightrightarrows E ; G \rightrightarrows M)$ be a VB-groupoid with (associated) $V B$-algebroid $(W \Rightarrow E ; A \Rightarrow M)$. The Van Est map 2.52) restricts to a cochain map

$$
\mathcal{V}:\left(\widehat{C}_{\mathrm{def}, \operatorname{lin}}^{\bullet}(\mathcal{W}), \delta\right) \rightarrow\left(C_{\mathrm{def}, \operatorname{lin}}^{\bullet}(W), \delta\right),
$$

where $\widehat{C}_{\text {def,lin }}^{\bullet}(\mathcal{W})$ denotes linear, normalized cochains, i.e. $\widehat{C}_{\operatorname{def}, \text { lin }}^{\bullet}(\mathcal{W})=\widehat{C}_{\text {def }}^{\bullet}(\mathcal{W}) \cap C_{\text {def,lin }}^{\bullet}(\mathcal{W})$.

Proof: When the side bundle $E$ has positive rank the proof can be significantly simplified by noticing that in this case a cochain $\tilde{c} \in C_{\mathrm{def}}^{\bullet}(W)$ is linear if and only if it preserves linear sections. This means that if rank $E>0$, only condition 2.56 is needed in the definition of linear cochains, while conditions 2.57)-2.61) are redundant. This can be seen, for instance, in local coordinates. In the rest of this proof we assume rank $E>0$. A complete proof will be presented in [40].

So, it is enough to show that if $\tilde{a}$ is a linear section of $W \rightarrow E$, then $R_{\tilde{a}} \operatorname{maps} C_{\operatorname{def}, l i n}^{\bullet+1}(\mathcal{W})$ to $\widehat{C}_{\text {def,lin }}^{\bullet}(\mathcal{W})$. But this immediately follows from 2.55 and the fact that from linearity the right invariant vector field $\overrightarrow{\vec{a}} \in \mathfrak{X}(\mathcal{W})$ is a linear vector field on $\mathcal{W}$ (Proposition 2.8), hence it generates a flow $\left\{\phi_{\varepsilon}^{\tilde{a}}\right\}_{\varepsilon}$ by vector bundle automorphisms.

\section{Multiplicative derivations of trivial-core VB-groupoids and alge- broids}

\subsection{VB-groupoids/algebroids and representations}

In this section we discuss the properties of some special class of VB-groupoids/algebroids. We have mentioned in the introduction that VB-groupoids/algebroids are intrinsic models of 2-term representations up to homotopy (see [27, 28], see also Section 4 for more details). For instance, the isomorphism class of the tangent VB-groupoid/algebroid corresponds to the isomorphism class of the adjoint representation (up to homotopy). In this picture, 2-term representations up to homotopy concentrated in one single degree $(-1$ or 0$)$ are genuine representations and correspond to the class of VB-groupoids/algebroids that we aim to discuss here.

Definition 3.1 (Trivial-core VB-groupoid) A VB-groupoid is trivial-core if its core is trivial.

Trivial-core VB-groupoids are also known as vacant. Let $\left(E_{G} \rightrightarrows E ; G \rightrightarrows M\right)$ be a trivial-core VB-groupoid. In this case, the source $\tilde{s}: E_{G} \rightarrow E$ is a regular vector bundle morphism (covering $s: G \rightarrow M)$. Hence $E_{G}$ is canonically isomorphic to $s^{*} E$ as a vector bundle over $G$. In the following we will understand the latter isomorphism and denote $E_{G}=s^{*} E$. 
Example 3.2 Let $G \rightrightarrows M$ be a Lie groupoid and let $E \rightarrow M$ be a vector bundle equipped with a left representation of $G$. Then there is a trivial core VB-groupoid structure $\left(E_{G}=s^{*} E \rightrightarrows E ; G \rightrightarrows M\right)$. The structure maps of the top groupoid $E_{G} \rightrightarrows E$ are the following:

i.) the source and target maps are given by $\tilde{s}(g, e)=e$ and $\tilde{t}(g, e)=g . e$, respectively,

ii.) the multiplication $\tilde{m}$ is given by $\tilde{m}\left((g, e),\left(g^{\prime}, e^{\prime}\right)\right)=\left(g g^{\prime}, e^{\prime}\right)$,

iii.) the unit and the inversion are given by $\tilde{u}(e)=\left(1_{q(e)}, e\right)$ and $\tilde{i}(g, e)=\left(g^{-1}, g . e\right)$.

In other words, $E_{G} \rightrightarrows E$ is the action groupoid corresponding to the action of $G$ on the fibered manifold $E \rightarrow M$. It is easy to see that every trivial-core VB-groupoid arises in this way. In 28 trivial-core VB-groupoids $\left(E_{G} \rightrightarrows E ; G \rightrightarrows M\right)$ correspond to representations of $G$ on $E$, seen as representations up to homotopy concentrated in degree 0 .

Definition 3.3 (Full-core VB-groupoid) A VB-groupoid ( $\mathcal{W} \rightrightarrows E ; G \rightrightarrows M)$ is full-core if its core is $C=\left.\mathcal{W}\right|_{M}$.

Let $(\mathcal{W} \rightrightarrows E ; G \rightrightarrows M)$ be a full-core VB-groupoid with core $C$. In this case, $E=0_{M}$, the rank 0 vector bundle over $M$, and $\mathcal{W}$ is canonically isomorphic to $t^{*} C$.

Example 3.4 Let $G \rightrightarrows M$ be a Lie groupoid and let $q: C \rightarrow M$ be a vector bundle carrying a left representation of $G$. Denote by $0_{M} \rightarrow M$ the rank zero vector bundle. Then there is a full-core VB-groupoid structure $\left(t^{*} C \rightrightarrows 0_{M} ; G \rightrightarrows M\right)$. The structure maps of the top groupoid $t^{*} C \rightrightarrows 0_{M}$ are the following:

i.) the source and the target are given by $\tilde{s}(g, \chi)=0_{s(g)}$ and $\tilde{t}(g, \chi)=0_{t(g)}=0_{q(\chi)}$,

ii.) the multiplication is given by $\tilde{m}\left((g, \chi),\left(g^{\prime}, \chi^{\prime}\right)\right)=\left(g g^{\prime}, \chi+g \cdot \chi^{\prime}\right)$,

iii.) the unit and the inversion are given by $\tilde{u}\left(0_{x}\right)=\left(1_{x}, 0_{x}\right)$, for all $x \in M$, and $\tilde{i}(g, \chi)=$ $\left(g^{-1},-g^{-1} \cdot \chi\right)$.

In other words, $t^{*} C \rightrightarrows 0_{M}=M$ is the representation groupoid corresponding to the representation $C$ of $G$. Actually, every full-core VB-groupoid arises in this way. In 28 full-core VB-groupoids correspond to representations of $G$ seen as representations up to homotopy concentrated in degree -1 .

Clearly, full-core VB-groupoids are dual to trivial-core VB-groupoids and vice-versa. If ( $E_{G} \rightrightarrows$ $E ; G \rightrightarrows M)$ is the trivial-core VB-groupoid corresponding to a left representation of $G$ on $E$, then the dual full-core VB-groupoid corresponds to the transpose representation of $G$ on $C=E^{*}$. Similar definitions exist in the realm of Lie algebroids. Specifically, we give the following

Definition 3.5 (Trivial-core and full-core VB-algebroids) A VB-algebroid ( $W \Rightarrow E ; A \Rightarrow M)$ is trivial-core if its core is trivial, and it is full-core if its core is $C=\left.W\right|_{M}$.

Example 3.6 Let $A \Rightarrow M$ be a Lie algebroid, denote by $p: A \rightarrow M$ the projection, and let $q: E \rightarrow M$ be a vector bundle carrying a representation of $A$. Denote by $\nabla$ the flat $A$-connection in $E$ defining the representation. For every section $a \in \Gamma(A)$ the operator $\nabla_{a}$ is a derivation of $E$. Hence, it corresponds to a linear vector field on $E$, that we denote by $X_{a}$. The vector field $X_{a}$ projects onto $\rho(a)$. It is easy to see that there is a trivial-core VB-algebroid structure $\left(E_{A}:=p^{*} E \Rightarrow E ; A \Rightarrow M\right)$. The structure maps of the top Lie algebroid $E_{A} \Rightarrow E$ are the following:

i.) the projection $\tilde{p}=p_{A}: E_{A} \rightarrow E$ is the one induced by $p$,

ii.) the anchor $\tilde{\rho}: E_{A} \rightarrow T E$ is given by $\tilde{\rho}\left(a_{x}, e\right)=\left(X_{a}\right)_{e}$ for all $a \in \Gamma(A), x \in M$, and $e \in E_{x}$,

iii.) the Lie bracket $[-,-]: \Gamma\left(E_{A}, E\right) \times \Gamma\left(E_{A}, E\right) \rightarrow \Gamma\left(E_{A}, E\right)$ is uniquely defined by the anchor and

$$
\left[q^{*} a, q^{*} b\right]=q^{*}[a, b],
$$

for all pull-back sections $q^{*} a, q^{*} b \in \Gamma\left(E_{A}, E\right)$, with $a, b \in \Gamma(A)$. 
In other words, $E_{A} \Rightarrow E$ is the action algebroid corresponding to the action of $A$ on the fibered manifold $E \rightarrow M$. Every trivial-core VB-algebroid arises in this way. In 27] trivial-core VBalgebroids $\left(E_{A} \Rightarrow E ; A \Rightarrow M\right)$ correspond to representations of $A$ on $E$ seen as representations up to homotopy concentrated in degree 0 .

Example 3.7 Let $A$ be as in Example 3.6 and let $C \rightarrow M$ be a vector bundle with a flat $A$-connection $\nabla$. Then there is a full-core VB-algebroid structure $\left(A \times{ }_{M} C \Rightarrow 0_{M} ; A \Rightarrow M\right)$. The structure maps of the top Lie algebroid $A \times{ }_{M} C \Rightarrow 0_{M}=M$ are the following:

i.) the projection $\tilde{p}: A \times{ }_{M} C \rightarrow 0_{M}$ is the obvious one,

ii.) the anchor $\tilde{\rho}: A \times{ }_{M} C \rightarrow T M$ is given by $\tilde{\rho}(a, e)=\rho(a)$, and

iii.) the Lie bracket

$$
[-,-]: \Gamma\left(A \times_{M} C, M\right) \times \Gamma\left(A \times_{M} C, M\right) \rightarrow \Gamma\left(A \times_{M} C, M\right)
$$

is given by

$$
\left[(a, \chi),\left(a^{\prime}, \chi^{\prime}\right)\right]=\left(\left[a, a^{\prime}\right], \nabla_{a} \chi^{\prime}-\nabla_{a^{\prime}} \chi\right)
$$

for all $a, a^{\prime} \in \Gamma(A)$, and $\chi, \chi^{\prime} \in \Gamma(C)$.

In other words, $A \times{ }_{M} C \rightarrow M$ is the representation algebroid corresponding to the representation $C$ of $A$, i.e. the semidirect product of $A$ and $C$. All full-core VB-algebroids arise in this way. In [27] they correspond to representations of $A$ seen as representations up to homotopy concentrated in degree -1 .

Full-core VB-algebroids are dual to trivial-core VB-algebroids and vice-versa. If $\left(E_{A} \Rightarrow E ; A \Rightarrow M\right)$ is the trivial-core VB-algebroid corresponding to a representation of $A$ on $E$, the dual full-core VBalgebroid corresponds to the transpose representation of $A$ on $C=E^{*}$.

The proof of the following proposition is straightforward.

\section{Proposition 3.8}

i.) Let $G$ be a Lie groupoid with Lie algebroid $A$, and let $E, C$ be representations of $G$ (hence of $A)$. The VB-algebroid of the trivial-core (resp. full-core) VB-groupoid determined by $G$ and $E$ (resp. $C$ ) is the trivial-core (resp. full-core) VB-algebroid corresponding to $A$ and $E$ (resp. $C$ ).

ii.) Let $A$ be an integrable Lie algebroid with source simply connected integration $G$, and let $E, C$ be representations of $A$, hence of $G$. The trivial-core (resp. full-core) VB-algebroid determined by $A$ and $E$ (resp. $C$ ) is integrable and its source simply connected integration is the trivial-core (resp. full-core) VB-groupoid determined by $G$ and $E$ (resp. $C$ ).

Example 3.9 A VB-groupoid $(\mathcal{W} \rightrightarrows E ; G \rightrightarrows M)$ (resp. a VB-algebroid $(W \Rightarrow E ; A \Rightarrow M)$ ) such that $\mathcal{W}$ (resp. $W$ ) is a line bundle over $G$ (resp. $A$ ) is necessarily either trivial-core or full-core.

\subsection{The gauge algebroid of trivial-core/full-core VB-groupoids/algebroids}

Let $G \rightrightarrows M$ be a Lie groupoid and let $q: E \rightarrow M$ be a vector bundle carrying a representation of $G$. As discussed in the previous section, starting with this data we can construct two VB-groupoids, a trivial-core one $\left(E_{G} \rightrightarrows E ; G \rightrightarrows M\right)$ and a full-core one $\left(t^{*} E \rightrightarrows 0_{M} ; G \rightrightarrows M\right)$. Actually, $E_{G}=s^{*} E \rightarrow$ $G$ and $t^{*} E \rightarrow G$ are canonically isomorphic as vector bundles over $G$ (see, e.g., 60]). The vector bundle isomorphism $\tau: E_{G} \rightarrow t^{*} E$ is given by

$$
\tau(g, e)=(g, g . e),
$$

for all $(g, e) \in G_{s} \times_{q} E=E_{G}$. Hence the gauge algebroids $\mathrm{D} E_{G}$ and $\mathrm{D} t^{*} E$ are canonically isomorphic as Lie algebroids over $G$. 
In this section, we show that the gauge algebroid $\mathrm{D} E_{G}$ fits itself in a VB-groupoid $\left(\mathrm{D} E_{G} \rightrightarrows\right.$ $\mathrm{D} E ; G \rightrightarrows M$ ) (Proposition 3.10). Using the isomorphism $\tau$ we immediately get that $\mathrm{D} t^{*} E$ fits in a VB-groupoid as well. In other words, the situation is basically the same for trivial-core and full-core VB-groupoids, exactly as one would expect from the fact that they are both equivalent to the same object: a representation of a Lie groupoid.

Proposition 3.10 There is a natural VB-groupoid structure $\left(\mathrm{D} E_{G} \rightrightarrows \mathrm{D} E ; G \rightrightarrows M\right)$ and a short exact sequence of VB-groupoids:

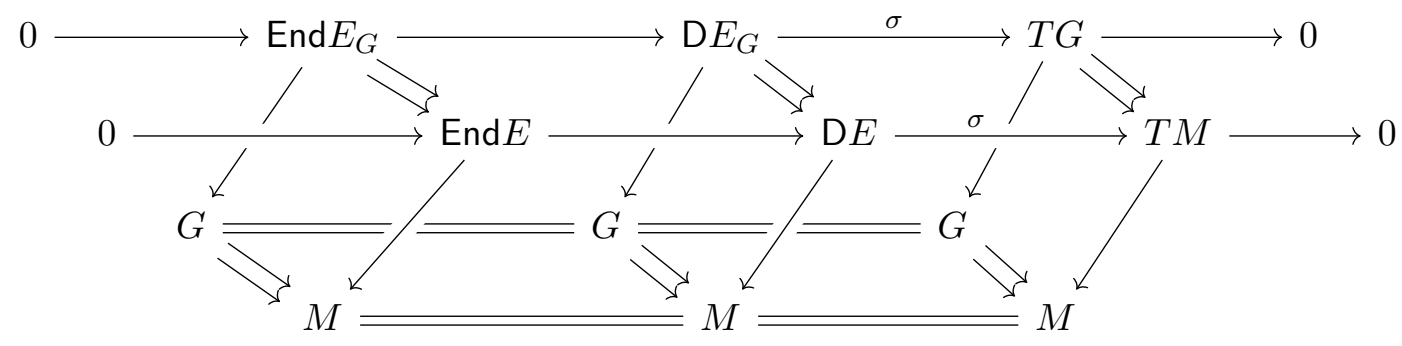

Here $\left(\operatorname{End} E_{G} \rightrightarrows \mathrm{End} E ; G \rightrightarrows M\right)$ is the trivial-core VB-groupoid corresponding to the induced action of $G$ on $\operatorname{End} E$.

Proof: Denote by

$$
\mathrm{a}: G_{s} \times_{q} E=E_{G} \rightarrow E, \quad(g, e) \mapsto \mathrm{a}_{g} e=g . e=\tilde{t}(g, e),
$$

the action of $G$ on $E$. We begin with a remark (for which we thank the referee): the action a induces an action $\mathcal{A}$ of the tangent groupoid $T G \rightrightarrows T M$ on $\sigma: \mathrm{D} E \rightarrow T M$. To see this, let $g \in G$, let $V \in T_{g} G$, and let $\Delta \in \mathrm{D}_{s(g)} E$ be such that:

$$
\mathrm{d} s(V)=\sigma(\Delta) .
$$

Choose a curve $\varepsilon \mapsto \Gamma(\varepsilon)$ in $G$ such that $\Gamma(0)=g$ and

$$
V=\left.\frac{d}{d \varepsilon}\right|_{\varepsilon=0} \Gamma(\varepsilon),
$$

and denote $\gamma=s \circ \Gamma$. Choose also a curve $\varepsilon \mapsto \gamma_{E}(\varepsilon): E_{s(g)} \rightarrow E_{\gamma(\varepsilon)}$ of isomorphisms such that

$$
\Delta=\left.\frac{d}{d \varepsilon}\right|_{\varepsilon=0} \gamma_{E}(\varepsilon)
$$

As the symbol of $\Delta$ is exactly $d s(V)$ this is possible. Now, put

$$
\mathcal{A}(V, \Delta):=\left.\frac{d}{d \varepsilon}\right|_{\varepsilon=0} \mathrm{a}_{\Gamma(\varepsilon)} \circ \gamma_{E}(\varepsilon) \circ \mathrm{a}_{g^{-1}} .
$$

It immediately follows that since a is an action, $\mathcal{A}$ is an action as well, as claimed.

Now, recall from Section 2.1 that the gauge algebroid $\mathrm{D} E_{N}$ of the pull-back vector bundle $E_{N}=$ $\phi^{*} E \rightarrow N$ along a smooth map $\phi: N \rightarrow M$, is (canonically isomorphic to) the pull-back $\mathrm{D} E_{N} \cong$ $T N_{\mathrm{d} \phi} \times_{\sigma} \mathrm{DE}$. In particular,

$$
\mathrm{D} E_{G} \cong T G_{\mathrm{d} s} \times_{\sigma} \mathrm{D} E .
$$

The action of $T G \rightrightarrows \mathrm{D} E$ then gives $\mathrm{D} E_{G}$ the structure of an action groupoid $\mathrm{D} E_{G} \rightrightarrows \mathrm{D} E$. It is now easy to check that with all its structure maps, $\left(\mathrm{D} E_{G} \rightrightarrows \mathrm{D} E ; G \rightrightarrows M\right)$ is indeed a VB-groupoid fitting in the exact sequence (3.5). We leave details to the reader (see also Remark 3.11). 
Remark 3.11 The structure maps of the groupoid $\mathrm{D} E_{G} \rightrightarrows \mathrm{D} E$ are those of the action groupoid. For instance, the source $s_{\mathrm{D}}: \mathrm{D} E_{G} \rightarrow \mathrm{D} E$ is just the projection of $\mathrm{D} E_{G}=T G_{\mathrm{ds}_{s} \times}{ }_{\sigma} \mathrm{D} E$ onto the second factor. In other words $s_{\mathrm{D}}=\mathrm{D} \tilde{s}$. The target $t_{\mathrm{D}}: \mathrm{D} E_{G} \rightarrow \mathrm{D} E$ is just the action map $\mathcal{A}: \mathrm{D} E_{G}=T G_{\mathrm{d} s \times}{ }_{\sigma} \mathrm{D} E \rightarrow \mathrm{D} E$, and a direct check exploiting 2.9) reveals that $t_{\mathrm{D}}=\mathrm{D} \tilde{t}$. It is easy to check that the other structure maps (multiplication $m_{\mathrm{D}}$, unit $u_{\mathrm{D}}$, invertion $i_{\mathrm{D}}$ ) are all induced by those of the VB-groupoid $\left(E_{G} \rightrightarrows E ; G \rightrightarrows M\right)$ via application of the functor D. The multiplication may need a little explanation. Consider the space $E_{G}^{(2)}=E_{G \tilde{s}} \times_{\tilde{t}} E_{G}$ of composable arrows of $E_{G} \rightrightarrows E$. It is a vector bundle over $G^{(2)}$ in the obvious way, and the projections $\tilde{p r}_{i}: E_{G}^{(2)} \rightarrow E_{G}$ over the factors are regular vector bundle morphisms covering the projections $\operatorname{pr}_{i}: G^{(2)} \rightarrow G, i=1,2$. Similarly, the multiplication $\tilde{m}: E_{G}^{(2)} \rightarrow E_{G}$ is a regular vector bundle morphism covering $m: G^{(2)} \rightarrow G$. The space of composable arrows of $\mathrm{D} E_{G} \rightrightarrows \mathrm{D} E$ is

$$
\left(\mathrm{D} E_{G}\right)^{(2)}=\mathrm{D} E_{G \mathrm{D} \tilde{s}} \times_{\mathrm{D} \tilde{t}} \mathrm{D} E_{G},
$$

and it is a vector bundle over $G^{(2)}$ as well. Finally, the map

$$
\mathrm{D}\left(E_{G}^{(2)}\right) \rightarrow\left(\mathrm{D} E_{G}\right)^{(2)}, \quad \square \mapsto\left(\mathrm{Dpr}_{1}(\square), \mathrm{Dpr}_{2}(\square)\right)
$$

is an isomorphism of vector bundles over $G^{(2)}$. Under this identification, $m_{\mathrm{D}}=\mathrm{D} \tilde{m}$. Similarly, $u_{\mathrm{D}}=\mathrm{D} \tilde{u}$ and $i_{\mathrm{D}}=\mathrm{D} \tilde{i}$. This shows a close analogy between the groupoid $\mathrm{D} E_{G} \rightrightarrows \mathrm{D} E$ and the tangent groupoid $T G \rightrightarrows T M$.

Remark 3.12 The core $C$ of the VB-groupoid $\left(\mathrm{D} E_{G} \rightrightarrows \mathrm{D} E ; G \rightrightarrows M\right)$ is canonically isomorphic to $A$. The isomorphism $A \rightarrow C$ maps $a$ to $\mathbb{D}_{a}$, where $\mathbb{D}$ is the obvious flat (ker ds)-connection in $E_{G}$.

A similar situation occurs for trivial-core/full-core VB algebroids: let $A \Rightarrow M$ be a Lie algebroid with projection $p: A \rightarrow M$, and let $E \rightarrow M$ be a vector bundle carrying a representation of $A$. We denote by $\nabla$ the flat $A$-connection in $E$. Beware that from now on $\mathrm{D} E_{A}$ will always indicate the gauge algebroid of the vector bundle $E_{A} \rightarrow A$ (not the vector bundle $E_{A} \rightarrow E$ ). We will now show that $\mathrm{D} E_{A}$ fits in a VB-algebroid ( $\left.\mathrm{D} E_{A} \Rightarrow \mathrm{D} E ; A \Rightarrow M\right)$ (Proposition 3.13). Finally, if $\left(E_{A} \Rightarrow E ; A \Rightarrow M\right)$ is the VB-algebroid of the VB-groupoid $\left(E_{G} \rightrightarrows E ; G \rightrightarrows M\right)$ then $\left(\mathrm{D} E_{A} \Rightarrow \mathrm{D} E ; A \Rightarrow M\right)$ is the VB-algebroid of ( $\left.\mathrm{D} E_{G} \rightrightarrows \mathrm{D} E ; G \rightrightarrows M\right)$ (Proposition 3.16).

Proposition 3.13 There is a natural VB-algebroid structure ( $\left.E_{A} \Rightarrow \mathrm{D} E ; A \Rightarrow M\right)$ and a short exact sequence of VB-algebroids:

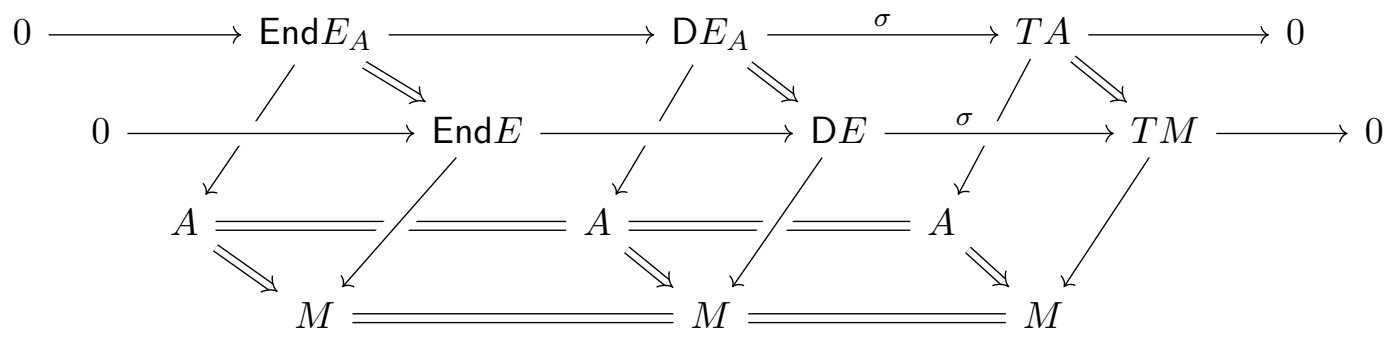

Here (End $\left.E_{A} \Rightarrow \operatorname{End} E ; A \Rightarrow M\right)$ is the trivial-core VB-algebroid corresponding to the induced action of $A$ on End $E$.

We propose a proof in the same spirit of that of Proposition 3.10. We conjecture that there exists a proof exploiting the fact that $\mathrm{D} E_{A}$ is the fat algebroid of the VB-algebroid $\left(T E_{A} \Rightarrow E_{A} ; T A \Rightarrow A\right)$. Actually, $T E_{A}$ is a triple structure: it is a Lie algebroid over $E_{A}$, over $T A$, and over $T E$, and all these structures are compatible. We expect the conjectured proof to require a preliminary analysis of the fat algebroid of a triple structure like that but this goes beyond the scopes of the present paper. 
ProOF: We begin remarking that similarly as in the groupoid case the flat connection $\nabla$ induces an (infinitesimal) action

$$
\mathfrak{A}: \Gamma(T A, T M) \rightarrow \mathfrak{X}(\mathrm{DE})
$$

of the tangent algebroid $T A \Rightarrow T M$ on $\sigma: \mathrm{D} E \rightarrow T M$. To see this, recall from Example 1.8, that sections of $T A \rightarrow T M$ are generated by core sections $\widehat{a}_{T}$, and linear sections of the form $\mathrm{d} a$, for some $a \in \Gamma(A)$. Now, every section $a$ of $A$ determines two vector fields on $\mathrm{D} E$ as follows. Correspondence $\Delta \mapsto\left[\nabla_{a}, \Delta\right]$ is a derivation of $\mathrm{D} E$. Hence, it corresponds to a (linear) vector field $Y_{a}$ on (the total space of) $\mathrm{D} E$ itself. Section $a$ does also determine another vector field on $\mathrm{D} E$ : the vertical lift $V_{a}$ of $\nabla_{a} \in \Gamma(\mathrm{D} E)$. We put

$$
\mathfrak{A}(\mathrm{d} a)=Y_{a} \quad \text { and } \quad \mathfrak{A}\left(\widehat{a}_{T}\right)=V_{a},
$$

for all $a \in \Gamma(A)$. It is easy to see that this uniquely defines an infinitesimal action as required.

Now, the gauge algebroid $\mathrm{D} E_{A}$ is the pull-back $\mathrm{D} E_{A} \cong T A_{\mathrm{d} p} \times_{\sigma} \mathrm{D} E$. The infinitesimal action $\mathfrak{A}$ then gives $\mathrm{D} E_{A}$ the structure of an action algebroid $\mathrm{D} E_{A} \Rightarrow \mathrm{D} E$. It is easy to check that $\left(\mathrm{D} E_{A} \Rightarrow\right.$ $\mathrm{D} E ; A \Rightarrow M$ ) with all its structure maps is indeed a VB-groupoid fitting in the exact sequence (3.14). We leave details to the reader (see also Remark 3.14).

Remark 3.14 The structure maps of the algebroid $\mathrm{D} E_{A} \Rightarrow \mathrm{D} E$ are those of the action algebroid. For instance, the projection $p_{\mathrm{D}}: \mathrm{D} E_{A} \rightarrow \mathrm{D} E$ is just the projection of $\mathrm{D} E_{A}=T A_{\mathrm{d} p} \times_{\sigma} \mathrm{D} E$ onto the second factor. In other words $p_{\mathrm{D}}=\mathrm{D} p$. In order to describe the anchor $\rho_{\mathrm{D}}: \mathrm{D} E_{A} \rightarrow T \mathrm{D} E$ first notice that sections of $\mathrm{D} E_{A} \rightarrow \mathrm{D} E$ are generated by pull-backs of sections of $T A \rightarrow T M$. The pull-back of a core section $\widehat{a}_{T}$ is a core section that we denote by $\widehat{a}_{\mathrm{D}}$, while the pull-back of a linear section of the form $\mathrm{d} a$ is simply $\mathrm{D} a, a \in \Gamma(A)$. Hence the anchor $\rho_{\mathrm{D}}: \mathrm{D} E_{A} \rightarrow T \mathrm{D} E$ is uniquely determined by

$$
\rho_{\mathrm{D}}(\mathrm{D} a)=Y_{a} \quad \text { and } \quad \rho_{\mathrm{D}}\left(\widehat{a}_{\mathrm{D}}\right)=V_{a},
$$

for all $a \in \Gamma(A)$. Finally,

$$
\begin{aligned}
& {[\mathrm{D} a, \mathrm{D} b]=\mathrm{D}[a, b],} \\
& {\left[\mathrm{D} a, \widehat{b}_{\mathrm{D}}\right]=\left[\widehat{a, b}_{\mathrm{D}},\right.} \\
& {\left[\widehat{a}_{\mathrm{D}}, \widehat{b}_{\mathrm{D}}\right]=0,}
\end{aligned}
$$

for all $a, b \in \Gamma(A)$.

Remark 3.15 The core $C$ of the VB-algebroid $\left(\mathrm{D} E_{A} \Rightarrow \mathrm{D} E ; A \Rightarrow M\right)$ is canonically isomorphic to $A$. The isomorphism $A \rightarrow C$ maps $a$ to $\mathbb{D}_{a}$, where $\mathbb{D}$ is the flat (ker $\left.\mathrm{d} p\right)$-connection in $E_{A}$.

Proposition 3.16 Let $\left(E_{G} \rightrightarrows E ; G \rightrightarrows M\right)$ be a trivial-core VB-groupoid, and let $\left(E_{A} \Rightarrow E ; A \Rightarrow M\right)$ be its trivial-core VB-algebroid (equivalently, let $\left(t^{*} E \rightrightarrows 0_{M} ; G \rightrightarrows M\right)$ be a full-core VB-groupoid and let $\left(A \times_{M} E \Rightarrow 0_{M} ; A \Rightarrow M\right)$ be its full-core VB-algebroid). Then $\left(\mathrm{D} E_{A} \Rightarrow \mathrm{D} E ; A \Rightarrow M\right)$ is canonically isomorphic to the VB-algebroid of the VB-groupoid $\left(\mathrm{D} E_{G} \rightrightarrows \mathrm{D} E ; G \rightrightarrows M\right)$.

Proof: It is enough to check that the Lie functor intertwines the action $\mathcal{A}$ in the proof of Proposition 3.10 and the infinitesimal action $\mathfrak{A}$ in the proof of Proposition 3.13 . To do this, denote by

$$
\dot{\mathcal{A}}: \Gamma(T A, T M) \rightarrow \mathfrak{X}(\mathrm{D} E)
$$

the infinitesimal action induced by $\mathcal{A}$. This means that for every section $w \in \Gamma(T A, T M)$, every $x \in M$ and every point $\Delta \in \mathrm{D}_{x} E$

$$
\dot{\mathcal{A}}(w)_{\Delta}:=\left.\frac{d}{d \lambda}\right|_{\lambda=0} \mathcal{A}\left(\phi_{\lambda}^{w}(v), \Delta\right)
$$


where $\left\{\phi_{\lambda}^{w}\right\}$ is the flow of $\vec{w}$, the right invariant vector field on $T G$ corresponding to $w, v=\sigma(\Delta)$, and we identify $v$ with $1_{v} \in T G$.

Now, we compute 3.20 in the cases $w=\widehat{a}_{T}$ and $w=\mathrm{d} a$, for some $a \in \Gamma(A)$. We begin with the first one. For every $x \in M$, and every $v \in T_{x} M$, we have (see [46])

$$
\phi_{\lambda}^{\widehat{a}_{T}}(v)=v+\lambda a_{x}
$$

Hence

$$
\begin{aligned}
\dot{\mathcal{A}}\left(\widehat{a}_{T}\right)_{\Delta} & =\left.\frac{d}{d \lambda}\right|_{\lambda=0} \mathcal{A}\left(v+\lambda a_{x}, \Delta\right)=\left.\frac{d}{d \lambda}\right|_{\lambda=0} \mathcal{A}\left(v+\lambda a_{x}, \Delta+0\right)=\left.\frac{d}{d \lambda}\right|_{\lambda=0} \mathcal{A}(v, \Delta)+\lambda \mathcal{A}\left(a_{x}, 0\right) \\
& =\left.\frac{d}{d \lambda}\right|_{\lambda=0} \Delta+\lambda \mathcal{A}\left(a_{x}, 0\right),
\end{aligned}
$$

where we used the (easy to check) fact that $\mathcal{A}: T G_{\mathrm{d} s} \times_{\sigma} \mathrm{D} E \rightarrow \mathrm{D} E$ is a vector bundle morphism covering the target $t: G \rightarrow M$. Now, for every section $e \in \Gamma(E)$,

$$
\nabla_{a_{x}} e=\left.\frac{d}{d \varepsilon}\right|_{\varepsilon=0} a_{\phi_{\varepsilon}^{a}(x)} e,
$$

where $\left\{\phi_{\varepsilon}^{a}\right\}$ is the flow of $\vec{a}$, and a direct computation exploiting (3.23) and (3.10 then shows that $\mathcal{A}\left(a_{x}, 0\right)=\nabla_{a_{x}}$. We conclude that

$$
\dot{\mathcal{A}}\left(\widehat{a}_{T}\right)_{\Delta}=\left.\frac{d}{d \lambda}\right|_{\lambda=0} \Delta+\lambda \nabla_{a_{x}}=\left(V_{a}\right)_{\Delta}=\mathfrak{A}\left(\widehat{a}_{T}\right)_{\Delta} .
$$

Finally, let $w=\mathrm{d} a$. We have

$$
\phi_{\lambda}^{\mathrm{d} a}=\mathrm{d} \phi_{\lambda}^{a},
$$

where, as usual, $\left\{\phi_{\lambda}^{a}\right\}$ is the flow of $\vec{a}$. Hence

$$
\dot{\mathcal{A}}(\mathrm{d} a)_{\Delta}:=\left.\frac{d}{d \lambda}\right|_{\lambda=0} \mathcal{A}\left(\mathrm{d} \phi_{\lambda}^{a}(v), \Delta\right) .
$$

Choose a curve $\varepsilon \mapsto \gamma(\varepsilon)$ in $G$ such that $\gamma(0)=x$, and

$$
v=\left.\frac{d}{d \varepsilon}\right|_{\varepsilon=0} \gamma(\varepsilon),
$$

and a curve $\varepsilon \mapsto \gamma_{E}(\varepsilon): E_{x} \rightarrow E_{\gamma(\varepsilon)}$ of isomorphisms such that

$$
\Delta=\left.\frac{d}{d \varepsilon}\right|_{\varepsilon=0} \gamma_{E}(\varepsilon)
$$

Then

$$
\mathrm{d} \phi_{\lambda}^{a}(v)=\left.\frac{d}{d \varepsilon}\right|_{\varepsilon=0} \phi_{\lambda}^{a}(\gamma(\varepsilon)),
$$

and from 3.10$)$,

$$
\mathcal{A}\left(\mathrm{d} \phi_{\lambda}^{a}(v), \Delta\right)=\left.\frac{d}{d \varepsilon}\right|_{\varepsilon=0} \mathrm{a}_{\phi_{\lambda}^{a}(\gamma(\varepsilon))} \circ \gamma_{E}(\varepsilon) \circ \mathrm{a}_{\phi_{\lambda}^{a}(x)^{-1}}=\mathrm{D} \Phi_{\lambda}^{a}(\Delta),
$$

where $\Phi_{\lambda}^{a}$ is the vector bundle isomorphism

$$
\Phi_{\lambda}^{a}: E \rightarrow E, \quad e \mapsto \mathrm{a}_{\phi_{\lambda}^{a}(q(e))} e .
$$

This shows that the vector field $\dot{\mathcal{A}}(\mathrm{d} a) \in \mathfrak{X}(\mathrm{D} E)$ generates the flow $\left\{\mathrm{D} \Phi_{\lambda}^{a}\right\}$. Notice that $\left\{\Phi_{\lambda}^{a}\right\}$ is the flow of $\nabla_{a}$. It now easily follows that $\left\{\mathrm{D} \Phi_{\lambda}^{a}\right\}$ is the flow of the adjoint operator $\left[\nabla_{a},-\right]$. In other words

$$
\dot{\mathcal{A}}(\mathrm{d} a)=Y_{a}=\mathfrak{A}(\mathrm{d} a) .
$$

This concludes the proof. 


\subsection{Multiplicative derivations as multiplicative sections}

Recall that a vector field $X$ on a Lie groupoid $G \rightrightarrows M$ is multiplicative if and only if, when regarded as a section $X: G \rightarrow T G$ of $T G \rightarrow G$, it is a morphism of Lie groupoids. A similar statement holds true for IM vector fields on Lie algebroids. In this section we prove similar results for multiplicative derivations of trivial-core and full-core VB-groupoids and IM derivations of trivial-core and full-core VB-algebroids.

Theorem 3.17 Let $\left(E_{G} \rightrightarrows E ; G \rightrightarrows M\right)$ be a trivial-core VB-groupoid. A derivation of $E_{G} \rightarrow G$ is multiplicative if and only if it is a multiplicative section of $\mathrm{D} E_{G} \rightarrow G$.

Proof: Let $G \rightrightarrows M$ be a Lie groupoid, and let $E \rightarrow M$ be a vector bundle equipped with a representation of $G$. Then $\left(E_{G} \rightrightarrows E ; G \rightrightarrows M\right)$ is a trivial-core VB-groupoid with structure maps $\tilde{s}, \tilde{t}, \tilde{m}, \tilde{u}, \tilde{i}$ as in Example 3.2. Similarly the dual representation of $G$ on $E^{*} \rightarrow M$ determines the trivial-core VB-groupoid $\left(E_{G}^{*} \rightrightarrows E^{*} ; G \rightrightarrows M\right)$ with structure maps denoted by $\tilde{s}^{\star}, \tilde{t}^{\star}, \tilde{m}^{\star}, \tilde{u}^{\star}, \tilde{i}^{\star}$. Applying Proposition 3.10 to both $\left(E_{G} \rightrightarrows E ; G \rightrightarrows M\right)$ and $\left(E_{G}^{*} \rightrightarrows E^{*} ; G \rightrightarrows M\right)$, we get VBgroupoids $\left(\mathrm{D} E_{G} \rightrightarrows \mathrm{D} E ; G \rightrightarrows M\right)$ and $\left(\mathrm{D} E_{G}^{*} \rightrightarrows \mathrm{D} E^{*} ; G \rightrightarrows M\right)$ with structure maps denoted by $s_{\mathrm{D}}, t_{\mathrm{D}}, m_{\mathrm{D}}, u_{\mathrm{D}}, i_{\mathrm{D}}$ and $s_{\mathrm{D}}^{\star}, t_{\mathrm{D}}^{\star}, m_{\mathrm{D}}^{\star}, u_{\mathrm{D}}^{\star}, i_{\mathrm{D}}^{\star}$ respectively. It is straightforward to see that isomorphism $\mathrm{D} E_{G} \rightarrow \mathrm{D} E_{G}^{*}, \Delta \mapsto \Delta^{*}$ (see Lemma 2.2 ) is actually a VB-groupoid isomorphism ( $\mathrm{D} E_{G} \rightrightarrows \mathrm{D} E ; G \rightrightarrows$ $M) \rightarrow\left(\mathrm{D} E_{G}^{*} \rightrightarrows \mathrm{D} E^{*} ; G \rightrightarrows M\right)$ (covering isomorphism $\left.\mathrm{D} E \rightarrow \mathrm{D} E^{*}\right)$. As a consequence, a derivation $\Delta \in \Gamma\left(\mathrm{D} E_{G}\right)$ is a multiplicative section of $\mathrm{D} E_{G} \rightarrow \mathrm{D} E$ if and only if its "dual" derivation $\Delta^{*} \in \Gamma\left(\mathrm{D} E_{G}^{*}\right)$ is a multiplicative section of $\mathrm{D} E_{G}^{*} \rightarrow \mathrm{D} E^{*}$. So it remains to check that the following conditions are equivalent:

i.) $X_{\Delta}$ is a multiplicative section of $T E_{G} \rightarrow E_{G}$,

ii.) $\Delta^{*}$ is a multiplicative section of $\mathrm{D} E_{G}^{*} \rightarrow G$.

First show that $i$.) $\Rightarrow$ ii.). Condition $i$.) means that there is a (necessarily unique) vector field $\underline{X} \in \mathfrak{X}(E)$ such that

$$
\mathrm{d} \tilde{s}\left(X_{\Delta}\right)_{(g, e)}=\underline{X}_{e}, \quad \mathrm{~d} \tilde{t}\left(X_{\Delta}\right)_{(g, e)}=\underline{X}_{g . e},
$$

for all $(g, e) \in E_{G}$, and, additionally, for every pair of composable arrows $\left((g, e),\left(g^{\prime}, e^{\prime}\right)\right) \in\left(E_{G}\right)^{(2)}$, we have

$$
\left(X_{\Delta}\right)_{\left(g g^{\prime}, e^{\prime}\right)}=d \tilde{m}\left(\left(X_{\Delta}\right)_{(g, e)},\left(X_{\Delta}\right)_{\left(g^{\prime}, e^{\prime}\right)}\right) .
$$

In this case, it follows from (3.33), that $\underline{X}$ is also a linear vector field, i.e. $\underline{X}=X_{\Delta}$ for some (necessarily unique) derivation $\underline{\Delta} \in \Gamma(\mathrm{D} E)$. Hence we can rephrase 3.33 in the following equivalent way

$$
s_{\mathrm{D}}^{\star} \Delta_{g}^{*}=\underline{\Delta}_{s(g)}^{*}, \quad t_{\mathrm{D}}^{\star} \Delta_{g}^{*}=\underline{\Delta}_{t(g)}^{*},
$$

for all $g \in G$. assume the first one of (3.33), then Equation (3.34) holds if and only if its sides have the same image through both $\mathrm{d} \tilde{s}$ and the tangent maps to the projections $q_{G}: E_{G} \rightarrow G$. The first condition is guaranteed by the first one of (3.33). As $X_{\Delta}$ is $q_{G}$-related to $\sigma(\Delta)=\sigma\left(\Delta^{*}\right)$, in view of (3.5) the second condition is equivalent to

$$
\sigma\left(\Delta_{g g^{\prime}}^{*}\right)=\sigma\left(m_{\mathrm{D}}^{\star}\left(\Delta_{g}^{*}, \Delta_{g^{\prime}}^{*}\right)\right)
$$

for all $\left(g, g^{\prime}\right) \in G^{(2)}$. Finally, from the isomorphism $\mathrm{D} E_{G}=T G_{\mathrm{d} s} \times{ }_{\sigma} \mathrm{D} E$, Equation (3.36), together with the first one of $(3.35)$, gives

$$
\Delta_{g g^{\prime}}^{*}=m_{\mathrm{D}}^{\star}\left(\Delta_{g}^{*}, \Delta_{g^{\prime}}^{*}\right) .
$$

for all $\left(g, g^{\prime}\right) \in G^{(2)}$. Equations (3.35) and (3.37) exactly mean that Condition ii.) holds. Implication ii.) $\Rightarrow i$.) can be proved by retracing the above steps in the reverse order. 
Theorem 3.18 Let $\left(t^{*} C \rightrightarrows 0_{M} ; G \rightrightarrows M\right)$ be a full-core VB-groupoid. A derivation of $t^{*} C \rightarrow G$ is multiplicative if and only if it corresponds, via the vector bundle isomorphism $\tau: C_{G} \rightarrow t^{*} C$, to a multplicative section of $\mathrm{D} C_{G} \rightarrow G$.

Proof: Let $\Delta$ be a derivation of $t^{*} C \rightarrow G$. Then $\Delta$ is multiplicative if and only if it generates a flow by automorphisms of $\left(t^{*} C \rightrightarrows 0_{M} ; G \rightrightarrows M\right)$ if and only if the dual derivation $\Delta^{*}$ generates a flow by automorphisms of the dual VB-groupoid $\left(C_{G}^{*} \rightrightarrows C^{*} ; G \rightrightarrows M\right)$ if and only if $\Delta^{*}$ is a multiplicative derivation of $C_{G}^{*} \rightarrow G$. Now $\left(C_{G}^{*} \rightrightarrows C^{*} ; G \rightrightarrows M\right)$ is a trivial-core VB-groupoid, hence $\Delta$ is multiplicative if and only if $\Delta^{*}$ is a multiplicative section of $\left(\mathrm{D} C_{G}^{*} \rightrightarrows \mathrm{D} C^{*} ; G \rightrightarrows M\right)$. Finally, recall from the proof of Theorem 3.17, that the correspondence $\Delta \mapsto \Delta^{*}$ is an isomorphism of the VB-groupoids $\left(\mathrm{DC}_{G} \rightrightarrows \mathrm{D} C ; G \rightrightarrows M\right)$ and $\left(\mathrm{DC}_{G}^{*} \rightrightarrows \mathrm{D} C^{*} ; G \rightrightarrows M\right)$. This concludes the present proof.

Theorem 3.19 Let $\left(E_{A} \Rightarrow E ; A \Rightarrow M\right)$ be a trivial-core VB-algebroid. A derivation of $E_{A} \rightarrow A$ is $I M$ if and only if it is an IM section of $\mathrm{D} E_{A} \rightarrow A$.

Proof: Let $\Delta$ be a derivation of $E_{A} \rightarrow A$ and let $X_{\Delta} \in \mathfrak{X}\left(E_{A}\right)$ be the corresponding linear vector field. Regard $\Delta$ as a section of $\mathrm{D} E_{A} \rightarrow A$, and $X_{\Delta}$ as a section of $E_{A} \rightarrow T E_{A}$. In view of Remark 2.19 it is enough to show that $\Delta$ is an IM section of $\mathrm{D} E_{A} \rightarrow A$ if and only if $X_{\Delta}$ is an IM section of $E_{A} \rightarrow T E_{A}$. This can be checked with a long, but straightforward, computation in local coordinates, that we sketch below. Let $\left(x^{i}\right)$ be local coordinates on $M$, let $\left(e_{A}\right)$ be a local basis of $\Gamma(E)$, and let $\left(v^{A}\right)$ be the associated, linear fiber coordinates on $E$. Similarly, let $\left(\varepsilon_{\alpha}\right)$ be a local basis of $\Gamma(A)$, and let $\left(u^{\alpha}\right)$ be the associated, linear, fiber coordinates on $A$. So $E_{A}$ is coordinatized by $\left(x^{i}, u^{\alpha}, v^{A}\right)$. A derivation $\Delta: \Gamma(E) \rightarrow E_{x}$ of $E \rightarrow M$ at the point $x \in M$, is locally of the form

$$
\Delta\left(f^{A} e_{A}\right)=\left(\left.\dot{x}^{i} \frac{\partial}{\partial x^{i}}\right|_{x} f^{B}+f^{A}(x) v_{A}^{B}\right) e_{B, x}
$$

for some real numbers $\left(\dot{x}^{i}, v_{A}^{B}\right)$ that serve as linear, fiber coordinates on the total space of $\mathrm{D} E \rightarrow M$. Note that $\sigma(\Delta)=\left.\dot{x}^{i} \frac{\partial}{\partial x^{i}}\right|_{x}$. Similarly, a derivation $\Delta: \Gamma\left(E_{A}\right) \rightarrow E_{x}$ of $E_{A} \rightarrow A$ at the point $a \in A$, is locally of the form

$$
\Delta\left(f^{A} p^{*} e_{A}\right)=\left(\left.\dot{x}^{i} \frac{\partial}{\partial x^{i}}\right|_{a} f^{B}+\left.\dot{u}^{\alpha} \frac{\partial}{\partial u^{\alpha}}\right|_{a} f^{B}+f^{A}(x) v_{A}^{B}\right) e_{B, x}
$$

for some real numbers $\left(\dot{x}^{i}, \dot{u}^{\alpha}, v_{A}^{B}\right)$ that serve as linear, fiber coordinates on the total space of $\mathrm{D} E_{A} \rightarrow$ $A$, and $\sigma(\Delta)=\left.\dot{x}^{i} \frac{\partial}{\partial x^{i}}\right|_{a}+\left.\dot{u}^{\alpha} \frac{\partial}{\partial u^{\alpha}}\right|_{a}$. The Lie algebroid structure in $A \rightarrow M$ is encoded by the de

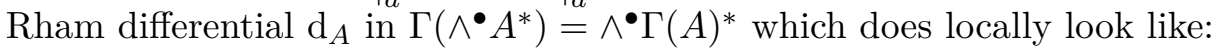

$$
\mathrm{d}_{A}=\rho_{\alpha}^{i} u^{\alpha} \frac{\partial}{\partial x^{i}}-\frac{1}{2} c_{\beta \gamma}^{\alpha} u^{\beta} \wedge u^{\gamma} \frac{\partial}{\partial u^{\alpha}},
$$

for some functions $\left(\rho_{\alpha}^{i}\right)$ encoding the anchor $\rho: A \rightarrow T M$, and some (structure) functions $\left(c_{\beta \gamma}^{\alpha}\right)$ encoding the Lie bracket in $\Gamma(A)$. Finally, the flat $A$-connection $\nabla$ in $E$ is locally encoded by "Christoffel symbols" $\left(\Gamma_{\alpha}^{B}\right)$ such that

$$
\nabla_{\varepsilon_{\alpha}} e_{A}=\Gamma_{\alpha A}^{B} e_{B} .
$$

It is now straightforward to check that the Lie algebroid structure in $E_{A} \rightarrow E$ is encoded by a de Rham differential $\mathrm{d}_{E_{A}}$ in $\wedge^{\bullet} \Gamma\left(E_{A}, E\right)^{*}$ locally given by:

$$
\mathrm{d}_{E_{A}}=\rho_{\alpha}^{i} u^{\alpha} \frac{\partial}{\partial x^{i}}-\Gamma_{\alpha A}^{B} v^{B} u^{\alpha} \frac{\partial}{\partial v^{B}}-\frac{1}{2} c_{\beta \gamma}^{\alpha} u^{\beta} \wedge u^{\gamma} \frac{\partial}{\partial u^{\alpha}} .
$$


The (tangent) Lie algebroid structure in $T E_{A} \rightarrow T E$ is then encoded by a differential $\mathrm{d}_{T E_{A}}$ in $\wedge \bullet \Gamma\left(T E_{A}, T E\right)^{*}$ locally given by the "tangent lift" of (3.42), i.e.

$$
\begin{aligned}
\mathrm{d}_{T E_{A}}= & \rho_{\alpha}^{i} u^{\alpha} \frac{\partial}{\partial x^{i}}+\left(\frac{\partial \rho_{\alpha}^{i}}{\partial x^{j}} \dot{x}^{j} u^{\alpha}+\rho_{\alpha}^{i} \dot{u}^{\alpha}\right) \frac{\partial}{\partial \dot{x}^{i}} \\
& -\Gamma_{\alpha A}{ }_{A}^{B} v^{B} u^{\alpha} \frac{\partial}{\partial v^{B}}-\left(\frac{\partial \Gamma_{\alpha B}^{A}}{\partial x^{i}} \dot{x}^{i} v^{B} u^{\alpha}+\Gamma_{\alpha B}{ }_{B}^{A} \dot{v}^{B} u^{\alpha}+\Gamma_{\alpha B}^{A} v^{B} \dot{u}^{\alpha}\right) \frac{\partial}{\partial \dot{v}^{A}} \\
& -\frac{1}{2} c_{\beta \gamma}^{\alpha} u^{\beta} \wedge u^{\gamma} \frac{\partial}{\partial u^{\alpha}}-\left(c_{\beta \gamma}^{\alpha} u^{\beta} \wedge \dot{u}^{\gamma}+\frac{1}{2} \frac{\partial c_{\beta \gamma}^{\alpha}}{\partial x^{i}} \dot{x}^{i} u^{\beta} \wedge u^{\gamma}\right) \frac{\partial}{\partial \dot{u}^{\alpha}} .
\end{aligned}
$$

Finally the Lie algebroid structure in $\mathrm{D} E_{A} \rightarrow \mathrm{D} E$ is encoded by a differential $\mathrm{d}_{\mathrm{D} E_{A}}$ in $\wedge^{\bullet} \Gamma\left(\mathrm{D} E_{A}, \mathrm{D} E\right)^{*}$ locally given by

$$
\begin{aligned}
\mathrm{d}_{\mathrm{D} E_{A}}= & \rho_{\alpha}^{i} u^{\alpha} \frac{\partial}{\partial x^{i}}+\left(\frac{\partial \rho_{\alpha}^{i}}{\partial x^{j}} \dot{x}^{j} u^{\alpha}+\rho_{\alpha}^{i} \dot{u}^{\alpha}\right) \frac{\partial}{\partial \dot{x}^{i}} \\
& +\left(\frac{\partial \Gamma_{\alpha B}^{A}}{\partial x^{i}} \dot{x}^{i} u^{\alpha}+\Gamma_{\alpha B}{ }_{B} v_{C}^{A} u^{\alpha}-\Gamma_{\alpha C}^{A} v_{B}^{C} u^{\alpha}+\Gamma_{\alpha B}^{A} \dot{u}^{\alpha}\right) \frac{\partial}{\partial v_{B}^{A}} \\
& -\frac{1}{2} c_{\beta \gamma}^{\alpha} u^{\beta} \wedge u^{\gamma} \frac{\partial}{\partial u^{\alpha}}-\left(c_{\beta \gamma}^{\alpha} u^{\beta} \wedge \dot{u}^{\gamma}+\frac{1}{2} \frac{\partial c_{\beta \gamma}^{\alpha}}{\partial x^{i}} \dot{x}^{i} u^{\beta} \wedge u^{\gamma}\right) \frac{\partial}{\partial \dot{u}^{\alpha}} .
\end{aligned}
$$

Now, let $\Delta$ be a section of $\mathrm{D} E_{A} \rightarrow A$ locally given by

$$
\Delta:\left\{\begin{array}{rl}
\dot{x}^{i} & =X^{i}(x, u) \\
\dot{u}^{\alpha} & =U^{\alpha}(x, u) \\
v_{B}^{A} & =V_{B}^{A}(x, u)
\end{array} .\right.
$$

The associated linear vector field on $E_{A}$ is the section $X_{\Delta}$ of $T E_{A} \rightarrow E_{A}$ locally given by

$$
X_{\Delta}:\left\{\begin{array}{rlc}
\dot{x}^{i} & = & X^{i}(x, u) \\
\dot{u}^{\alpha} & = & U^{\alpha}(x, u) \\
\dot{v}^{A} & = & -V_{B}^{A}(x, u) v^{B}
\end{array} .\right.
$$

By definition, $\Delta$ is an IM section if and only if

$(I M 1) \Delta$ is a morphism of the vector bundles $A \rightarrow M$ and $\mathrm{D} E_{A} \rightarrow \mathrm{D} E$, and

$(I M 2) \Delta^{\star} \circ \mathrm{d}_{\mathrm{D} E_{A}}=\mathrm{d}_{A} \circ \Delta^{\star}$,

where $\Delta^{\star}: \wedge^{\bullet} \Gamma\left(\mathrm{D} E_{A}, \mathrm{D} E\right)^{*} \rightarrow \wedge^{\bullet} \Gamma(A)^{*}$ is the obvious graded algebra map induced by $\Delta$ in view of (IM1). Similarly, $X_{\Delta}$ is an IM section if and only if

$\left(I M^{\prime} 1\right) X_{\Delta}$ is a morphism of the vector bundles $E_{A} \rightarrow E$ and $T E_{A} \rightarrow T E$, and

$\left(I M^{\prime} 2\right) X_{\Delta}^{\star} \circ \mathrm{d}_{T E_{A}}=\mathrm{d}_{E_{A}} \circ X_{\Delta}^{\star}$,

where $X_{\Delta}^{\star}: \wedge^{\bullet} \Gamma\left(T E_{A}, T E\right)^{*} \rightarrow \wedge^{\bullet} \Gamma\left(E_{A}, E\right)^{*}$ is the graded algebra map induced by $X_{\Delta}$ in view of $\left(I M^{\prime} 1\right)$. A direct computation shows that $(I M 1)$ and $\left(I M^{\prime} 1\right)$ are both equivalent to

$$
\begin{aligned}
X^{i} & =X^{i}(x), \\
V_{B}^{A} & =V_{B}^{A}(x), \\
U^{\alpha} & =U_{\beta}^{\alpha}(x) u^{\beta} .
\end{aligned}
$$


Finally, $\left(I M^{\prime} 1\right)$ and $\left(I M^{\prime} 2\right)$ are both equivalent to the following system of PDEs:

$$
\begin{aligned}
\rho_{\alpha}^{j} \frac{\partial X^{i}}{\partial x^{j}} & =\frac{\partial \rho_{\alpha}^{i}}{\partial x^{i}} X^{i}+\rho_{\beta}^{i} U_{\alpha}^{\beta}, \\
\rho_{\alpha}^{i} \frac{\partial V_{B}^{A}}{\partial x^{i}} & =\frac{\partial \Gamma_{\alpha B}^{A}}{\partial x^{i}} X^{i}+\Gamma_{\alpha B}^{C} V_{C}^{A}-\Gamma_{\alpha C}^{A} V_{B}^{C}+\Gamma_{\beta}{ }_{A}^{B} U_{\alpha}^{\beta}, \\
\rho_{\beta}^{i} \frac{\partial U_{\gamma}^{\alpha}}{\partial x^{i}}-\rho_{\gamma}^{i} \frac{\partial U_{\beta}^{\alpha}}{\partial x^{i}} & =c_{\delta \beta}^{\alpha} U_{\gamma}^{\delta}-c_{\delta \gamma}^{\alpha} U_{\beta}^{\delta}+c_{\beta \gamma}^{\delta} U_{\delta}^{\alpha}-\frac{\partial c_{\beta \gamma}^{\alpha}}{\partial x^{i}} X^{i} .
\end{aligned}
$$

This concludes the proof.

By an analogous computation we can prove the following

Theorem 3.20 Let $\left(A \times{ }_{M} C \Rightarrow 0_{M} ; A \Rightarrow M\right)$ be a full-core VB-algebroid. A derivation of $A \times{ }_{M} C \rightarrow$ $A$ is IM if and only if it is an IM section of $\mathrm{D} C_{A} \rightarrow A$.

We showed in this section that the gauge algebroid of a trivial-core or a full-core VB-groupoid is a VB-groupoid itself and likewise for VB-algebroids. For a generic VB-groupoid ( $\mathcal{W} \rightrightarrows E ; G \rightrightarrows M)$ the gauge algebroid is not a VB-groupoid. To see this notice that $\mathrm{DW}$ contains End $\mathcal{W}=\mathcal{W}^{*} \otimes \mathcal{W}$ as a vector subbundle (and a Lie subalgebroid). But the tensor product of two VB-groupoids over $G \rightrightarrows M$ is not a VB-groupoid in general. In fact it is part of a higher VB-groupoid or, more precisely, a simplicial vector bundle over the nerve of $G[19$. We conjecture that DW is a higher VB-groupoid in the same sense. In the case of a VB-algebroid $(W \Rightarrow E ; A \Rightarrow M)$ we can say a little bit more. The Lie algebroid $A \Rightarrow M$ is equivalent to the degree $1 \mathbb{N} Q$-manifold $\left(A[1], \mathrm{d}_{A}\right)$. Similarly, as proved by T. Voronov [62], $(W \Rightarrow E ; A \Rightarrow M)$ is equivalent to the vector bundle $\left(W[1]_{E} \rightarrow A[1], d_{W}\right)$ in the category of degree $1 \mathbb{N} Q$-manifolds. Here $W[1]_{E}$ is obtained from $W$ shifting by one the degree in the fibers over $E$. Now, not only $d_{W}$ is a homological vector field, but it is a linear vector field, hence it corresponds to a degree 1 derivation $\Delta_{W}$ of $W[1]_{E} \rightarrow A[1]$, such that $\Delta_{W}^{2}=0$. Denote by $\mathcal{D} W[1]_{E}$ the module of all graded derivations of $W[1]_{E} \rightarrow A[1]$, so that $\left.\left(\mathcal{D} W[1]_{E},\left[\Delta_{W},-\right]\right)\right)$ is a DG Lie algebra. More precisely it is the DG Lie algebra of sections of a DG Lie algebroid $\mathrm{D}_{A} W[1]_{E} \Rightarrow A[1]$. The cochain complex $\left.\left(\mathcal{D} W[1]_{E},\left[\Delta_{W},-\right]\right)\right)$ is canonically isomorphic to the linear deformation complex of $(W \Rightarrow E ; A \Rightarrow M)$ 24, 39], so that IM derivations of $W \rightarrow A$ are the same as degree zero derivations of $W[1]_{E} \rightarrow A[1]$ commuting with $\Delta_{W}$. In this way we encoded IM derivations in $\mathrm{D}_{A} W[1]_{E} \Rightarrow A[1]$. After a suitable shift, the latter is itself a vector bundle in the category of $\mathbb{N} Q$-manifolds, however it is not of degree 1, but of degree 2. So it is a degree 2 Lie algebroid module in the sense of Vauntrob [57 and corresponds to a 3-term representation up to homotopy of $A$ (see [51], see also next section, for more details on representations up to homotopy).

\section{A 2-term representation up to homotopy from a plain represen- tation}

\subsection{VB-groupoids}

There is a nice relationship between VB-groupoids and representations up to homotopy 28. Let $G \rightrightarrows M$ be a Lie groupoid. Recall that a representation up to homotopy of $G[2]$ is a graded vector bundle $q_{\varepsilon}: \mathcal{E}=\bigoplus_{k} \mathcal{E}_{k} \rightarrow M$ together with a degree $1, \mathbb{R}$-linear operator

$$
D: C^{\bullet}(G, \mathcal{E}) \rightarrow C^{\bullet}(G, \mathcal{E})
$$

satisfying

$$
D^{2}=0, \quad \text { and } \quad D(\omega \cdot \varepsilon)=\delta \omega \cdot \varepsilon+(-)^{\omega} \omega \cdot D \varepsilon,
$$


for all $\omega \in C^{\bullet}(G)$, and all $\varepsilon \in C^{\bullet}(G, \mathcal{E})$. Here $\left(C^{\bullet}(G), \delta\right)$ is the Lie groupoid complex of $G$, and $C^{p}(G, \mathcal{E})$ consists of $\mathcal{E}$-valued groupoid $p$-cochains, i.e. maps

$$
\varepsilon: G^{(p)} \rightarrow \mathcal{E}, \quad\left(g_{1}, \ldots, g_{p}\right) \mapsto \omega\left(g_{1}, \ldots, g_{p}\right) \in \mathcal{E}_{t\left(g_{1}\right)} .
$$

Additionally, $D$ is required to preserve normalized conchains, i.e. cochains vanishing when one of their entry is 1 . We stress that when we say that the degree of $D$ is 1 , we refer to the total degree, i.e. the sum of the cochain degree and the degree in $\mathcal{E}$. For more details see $[2$. We are mainly interested in 2-term representations up to homotopy, i.e. to the case when $\mathcal{E}=\mathcal{E}_{-1} \oplus \mathcal{E}_{0}$ is concentrated in degree $-1,0$. A 2 -term representation up to homotopy $\left(\mathcal{E}=\mathcal{E}_{-1} \oplus \mathcal{E}_{0}, D\right)$ is actually equivalent to the following data

- a vector bundle map $\partial: \mathcal{E}_{-1} \rightarrow \mathcal{E}_{0}$,

- $G$-quasi-actions on $\mathcal{E}_{-1}$ and $\mathcal{E}_{0}$, i.e. vector bundle maps $\Delta_{-1}: G_{s} \times_{q_{\varepsilon}} \mathcal{E}_{-1} \rightarrow \mathcal{E}_{-1}$ and $\Delta_{0}: G_{s} \times_{q_{\varepsilon}} \varepsilon_{0} \rightarrow \mathcal{E}_{0}$ covering the target map $t: G \rightarrow M$,

- a transformation 2-cochain $\mathcal{W} \in C^{2}\left(G, \mathcal{E}_{-1} \rightarrow \mathcal{E}_{0}\right)$, i.e. a vector bundle map

$$
\varpi: G_{s \mathrm{pr}_{1}}^{(2)} \times_{q_{\varepsilon}} \mathcal{E}_{-1} \rightarrow \mathcal{E}_{0}, \quad\left(g_{1}, g_{2} ; v\right) \mapsto \varpi_{\left(g_{1}, g_{2}\right)} v \in E_{t\left(g_{2}\right)}
$$

where $s \operatorname{pr}_{1}: G^{(2)} \rightarrow M$ is the composition of the first projection $\operatorname{pr}_{1}: G^{(2)} \rightarrow G$ followed by the source map.

Additionally data $\partial, \Delta_{-1}, \Delta_{0}, \varpi$ have to satisfy suitable compatibility conditions [28, Section 2.7].

The main remark here is that VB-groupoids $(\mathcal{W} \rightrightarrows E ; G \rightrightarrows M)$ are equivalent to 2-term representations up to homotopy of the Lie groupoid $G \rightrightarrows M$ up to isomorphisms. From ( $\mathcal{W} \rightrightarrows E ; G \rightrightarrows M)$ one can construct the corresponding (isomorphism class of) 2-term representation(s) up to homotopy as follows. As usual, denote by $C$ the core. There is a short exact sequence of vector bundles over $G$

$$
0 \rightarrow t^{*} C \rightarrow \mathcal{W} \rightarrow s^{*} E \rightarrow 0 .
$$

where the inclusion $t^{*} C \rightarrow \mathcal{W}$ is defined by $(g, \chi) \mapsto \chi \cdot 0_{g}$, and the projection $\mathcal{W} \rightarrow s^{*} E$ is the obvious one. Next we need a horizontal lift, i.e. a splitting $h: s^{*} E \rightarrow \mathcal{W},(g, e) \mapsto h_{g} e$ of (4.5) with the additional property of being compatible with the units, i.e. $h\left(1_{x}, e\right)=1_{e}$ for all $e \in E_{x}, x \in M$. Horizontal lifts always exist. Now put $\mathcal{E}_{-1}=C[1]$ (where the "[1]" refers to the fact that sections of $C$ should be thought as possessing degree -1$), \mathcal{E}_{0}=E$ and finally

$$
\begin{aligned}
\partial c & =\tilde{t}(c) \\
\Delta_{-1}(g, c) & =h_{g}(\tilde{t}(c)) \cdot c \cdot 0_{g^{-1}} \\
\Delta_{0}(g, e) & =\tilde{t}\left(h_{g}(e)\right) \\
\varpi_{\left(g_{1}, g_{2}\right)} e^{\prime} & =\left(h_{g_{1} g_{2}}\left(e^{\prime}\right)-h_{g_{1}}\left(\tilde{t}\left(h_{g_{2}}\left(e^{\prime}\right)\right)\right) \cdot h_{g_{2}}\left(e^{\prime}\right)\right) \cdot 0_{\left(g_{1} g_{2}\right)^{-1}}
\end{aligned}
$$

for all $g \in G, c \in C_{s(g)}, e \in E_{s(g)},\left(g_{1}, g_{2}\right) \in G^{(2)}$, and $e^{\prime} \in E_{s\left(g_{1}\right)}$. Then data $\left(\partial, \Delta_{-1}, \Delta_{0}, \varpi\right)$ define a 2-term representation up to homotopy $\left(\mathcal{E}=\mathcal{E}_{-1} \oplus \mathcal{E}_{0}, D\right)$ which is actually independent of $h$ up to isomorphisms (see 28 for more details).

In the last part of this subsection we describe the data $\left(\partial, \Delta_{-1}, \Delta_{0}, \varpi\right)$ for the 2-term representation up to homotopy corresponding to the VB-groupoid ( $\mathrm{D} E_{G} \rightrightarrows \mathrm{D} E ; G \rightrightarrows M$ ) from Proposition 3.10. So let $G \rightrightarrows M$ be a Lie groupoid, and let $E$ be a representation of it. Recall from Remark 3.12 that the core of $\left(\mathrm{D} E_{G} \rightrightarrows \mathrm{D} E ; G \rightrightarrows M\right)$ is canonically isomorphic to the Lie algebroid $A$ of $G$. The isomorphism identifies $a$ to $\mathbb{D}_{a}$, where $\mathbb{D}$ is the flat (ker $\mathrm{d} s$ )-connection in $E_{G}$. So there is a short exact sequence of vector bundles over $G$

$$
0 \rightarrow t^{*} A \rightarrow \mathrm{D} E_{G} \rightarrow s^{*} \mathrm{D} E \rightarrow 0,
$$


where the inclusion $t_{\mathrm{D}}^{*} A \rightarrow \mathrm{D} E_{G}$ is given by

$$
(g, a)=\mathbb{D}_{a} \cdot 0_{g}=\mathbb{D}_{\vec{a}_{g}} .
$$

As $G$ acts on $E$, then $A$ acts on $E$ as well, and we denote by $\nabla: A \rightarrow \mathrm{D} E$ the corresponding flat $A$-connection. Now we choose a horizontal lift $h: s^{*} \mathrm{D} E \rightarrow \mathrm{D} E_{G}$, to get a 2-term representation up to homotopy $(\mathcal{E}, D)$ of $G$, with $\mathcal{E}=A[1] \oplus \mathrm{D} E$. Specializing formulas (4.6) to the present situation it is easy to see that $D$ corresponds to the data $\left(\partial, \Delta_{-1}, \Delta_{0}, \varpi\right)$ given by

$$
\begin{aligned}
\partial a & =\nabla_{a} \\
\Delta_{-1}(g, a) & =m_{\mathrm{D}}\left(h_{g}\left(\nabla_{a}\right), \mathbb{D}_{\vec{a}_{g^{-1}}}\right) \\
\Delta_{0}(g, \square) & =t_{\mathrm{D}}\left(h_{g}(\square)\right) \\
\varpi_{\left(g_{1}, g_{2}\right)} \square^{\prime} & =\mathrm{D} R_{\left(g_{1} g_{2}\right)^{-1}}\left(h_{g_{1} g_{2}}\left(\square^{\prime}\right)-m_{\mathrm{D}}\left(h_{g_{1}}\left(t_{\mathrm{D}}\left(h_{g_{2}}\left(\square^{\prime}\right)\right)\right), h_{g_{2}}\left(\square^{\prime}\right)\right)\right)
\end{aligned}
$$

for all $g: x \rightarrow y \in G, a \in A_{s(g)}, \square \in \mathrm{D}_{s(g)} E,\left(g_{1}, g_{2}\right) \in G^{(2)}$, and $\square^{\prime} \in \mathrm{D}_{s\left(g_{1}\right)} E$, where $R_{g}$ : $s^{-1}(y) \times E_{y} \rightarrow s^{-1}(x) \times E_{x}$ is the right translation.

Remark 4.1 Let $\pi: \mathrm{D} E_{G} \rightarrow t^{*} A$, be the projection induced by the horizontal lift $h$. It is not hard to show that quasi-action $\Delta_{-1}$ is equivalently given by

$$
\Delta_{-1}(g, a)=-\pi_{g}\left(\mathrm{D} L_{g} \circ \mathrm{D} i\left(\mathbb{D}_{a}\right)\right),
$$

for all $g: x \rightarrow y \in G, a \in A_{s(g)}$, where $L_{g}: t^{-1}(x) \times E_{x} \rightarrow t^{-1}(y) \times E_{y}$ is the left translation, and $i: s^{-1}(x) \times E_{x} \rightarrow t^{-1}(x) \times E_{x}$ is the inversion. Formula 4.10 is the "derivation version" of the $G$-quasi-action on $T M$ determined by an Ehresmann connection in $G$, entering the adjoint representation (up to homotopy) of $G$, and appearing in [2, Definition 2.11].

Summarizing, we proved the following

Proposition 4.2 Let $G \rightrightarrows M$ be a Lie groupoid and let $E$ be a representation of $G$. Additionally, let $h: s^{*} \mathrm{D} E \rightarrow \mathrm{D} E_{G}$ be a horizontal lift. These data define a 2-term representation up to homotopy $(\mathcal{E}, D)$ of $G$, where $\mathcal{E}=A[1] \oplus \mathrm{DE}$, and $D$ corresponds to data $\left(\partial, \Delta_{-1}, \Delta_{0}, \varpi\right)$ given by (4.9). The representation up to homotopy $(\mathcal{E}, D)$ does only depend on $E$, in particular it is independent of $h$, up to isomorphisms.

Remark 4.3 Using the isomorphism $\mathrm{D} E_{G} \cong T G_{d s} \times{ }_{\sigma} \mathrm{D} E$, a horizontal lift $h: s^{*} \mathrm{D} E \rightarrow \mathrm{D} E_{G}$, can be thought of as a morphism $s^{*} \mathrm{D} E \rightarrow T G$ of vector bundles over $G$, additionally covering $\sigma: \mathrm{D} E \rightarrow T M$. The 4.9 can then be re-expressed in terms of the action $\mathcal{A}$ of $T G$ on $\mathrm{DE}$. We leave the details to the reader.

Remark 4.4 The symbol, relates the VB-groupoids ( $\left.\mathrm{D} E_{G} \rightrightarrows \mathrm{D} E ; G \rightrightarrows M\right)$ and $(T G \rightrightarrows T M ; G \rightrightarrows$ $M)$ (Diagram 3.5). In turn, a horizontal lift $k: s^{*} T M \rightarrow T G$ of the tangent groupoid determines a 2term representation up to homotopy $\left(\mathcal{E}^{T}, D^{T}\right)$ of $G$ where $\mathcal{E}^{T}=A[1] \oplus T M$, the adjoint representation (see $[2]$ for details, see also [28]). It is now natural to expect that the symbol relates $(\mathcal{E}, D)$ and $\left(\varepsilon^{T}, D^{T}\right)$. This is indeed the case if the horizontal lifts $h: s^{*} \mathrm{D} E \rightarrow \mathrm{D} E_{G}$ and $k: s^{*} T M \rightarrow T G$ are chosen in an appropriate way. Namely, let $h, k$ be such that the diagram

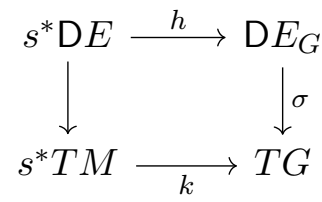


commutes (here the left vertical arrow is that induced by the symbol $\mathrm{D} E \rightarrow T M$ ). Such $h, k$ indeed exist. To see this, choose $k$ and use the isomorphism $\mathrm{D} E_{G} \cong T G_{d s} \times_{\sigma} \mathrm{D} E$ to put

$$
h(g, \square):=(k(g, \sigma(\square)), \square) .
$$

The $h, k$ have all the required properties. Finally, denote by $\left(\partial^{T}, \Delta_{-1}^{T}, \Delta_{0}^{T}, \varpi^{T}\right)$ the structure data of the adjoint representation. It immediately follows from Diagrams (3.5) and (4.11) that

$$
\sigma \circ \partial=\partial^{T}, \quad \Delta_{-1}=\Delta_{-1}^{T}, \quad \sigma \circ \Delta_{0}=\Delta_{0}^{T} \circ\left(\operatorname{id}_{G} \times \sigma\right), \quad \text { and } \quad \sigma \circ \varpi=\varpi^{T} .
$$

In other words, the homomorphism of $C^{\bullet}(G)$-modules

$$
C^{\bullet}(G, A[1] \oplus \mathrm{D} E) \rightarrow C^{\bullet}(G, A[1] \oplus T M)
$$

obtained from $\operatorname{id}_{A} \oplus \sigma: A[1] \oplus \mathrm{D} E \rightarrow A[1] \oplus T M$ by extension of scalars, is a DG-module homomorphism, i.e. it additionally intertwines the differentials $D$ and $D^{T}$.

\subsection{VB-algebroids}

We recall again from 27 that there is a nice relationship between VB-algebroids and representations up to homotopy. Let $A \Rightarrow M$ be a Lie algebroid. Recall that a representation up to homotopy of $A[1]$ is a graded vector bundle $\mathcal{E} \rightarrow M$ together with a degree $1, \mathbb{R}$-linear operator

$$
D: \Omega(A, \mathcal{E}) \rightarrow \Omega(A, \mathcal{E})
$$

satisfying

$$
D^{2}=0, \quad \text { and } \quad D(\omega \cdot \varepsilon)=\mathrm{d}_{A} \omega \cdot \varepsilon+(-)^{\omega} \omega \cdot D \varepsilon,
$$

for all $\omega \in \Omega(A)$, and all $\varepsilon \in \Omega(A, \mathcal{E})$ (i.e., $D$ is an homological derivation of the graded $\Omega(A)$ module $\Omega(A, E)$, with symbol $\left.\mathrm{d}_{A}\right)$. Here $\left(\Omega(A)=\Gamma\left(\wedge^{\bullet} A^{*}\right), \mathrm{d}_{A}\right)$ is the de Rham complex of $A$, and $\Omega(A, \mathcal{E})=\Gamma\left(\wedge^{\bullet} A^{*} \otimes \mathcal{E}\right)$. We are mainly interested in 2-term representations up to homotopy, i.e. to the case when $\mathcal{E}=\mathcal{E}_{-1} \oplus \mathcal{E}_{0}$ is concentrated in degree -1,0. A 2-term representation up to homotopy $\left(\mathcal{E}=\mathcal{E}_{-1} \oplus \mathcal{E}_{0}, D\right)$ is equivalent to the following data

- a vector bundle map $\partial: \mathcal{E}_{-1} \rightarrow \mathcal{E}_{0}$,

- an $A$-connection $\nabla_{-1}$ in $\mathcal{E}_{-1}$, with curvature $R_{-1} \in \Omega^{2}\left(A\right.$, End $\left.\mathcal{E}_{-1}\right)$,

- an $A$-connection $\nabla_{0}$ in $\mathcal{E}_{0}$, with curvature $R_{0} \in \Omega^{2}\left(A\right.$, End $\left.\varepsilon_{0}\right)$ and

- a $\operatorname{Hom}\left(\mathcal{E}_{0}, \mathcal{E}_{-1}\right)$-valued 2-form $R \in \Omega^{2}\left(A, \operatorname{Hom}\left(\mathcal{E}_{0}, \mathcal{E}_{-1}\right)\right)$,

such that

$$
\partial \circ\left(\nabla_{-1}\right)_{a}=\left(\nabla_{0}\right)_{a} \circ \partial, \quad R_{-1}(a, b)=R(a, b) \circ \partial, \quad R_{0}(a, b)=\partial \circ R(a, b), \quad \text { and } \quad d_{\nabla_{\text {Hom }}} R=0,
$$

for all $a, b \in \Gamma(A)$, where $\nabla^{\text {Hom }}$ is the $A$-connection in $\operatorname{Hom}\left(\varepsilon_{0}, \mathcal{E}_{-1}\right)$ induced by $\nabla_{-1}, \nabla_{0}$. The data $\left(\partial, \nabla_{-1}, \nabla_{0}, R\right)$ correspond to the representation up to homotopy whose differential $D$ is

$$
D=\partial+\nabla_{-1}+\nabla_{0}+R
$$

where we interpret $\partial, \nabla_{-1}, \nabla_{0}, R$ as degree 1 operators on $\Omega\left(A, \mathcal{E}_{-1} \oplus \mathcal{E}_{0}\right)$ in the obvious way (see 27] for more details).

The main remark here is that VB-algebroids ( $W \Rightarrow E ; A \Rightarrow M)$ are equivalent to 2-term representations up to homotopy of a Lie algebroid $A \Rightarrow M$ up to isomorphisms. From $(W \Rightarrow E ; A \Rightarrow M)$ 
one can construct the corresponding (isomorphism class of) 2-term representation(s) up to homotopy as follows. As usual, denote by $C$ the core and by $\widehat{A}$ the fat algebroid, and consider the short exact sequence

$$
0 \rightarrow \operatorname{Hom}(E, C) \rightarrow \widehat{A} \rightarrow A \rightarrow 0 .
$$

Choose a splitting $\sigma: A \rightarrow \widehat{A}$. Now put $\mathcal{E}_{-1}=C[1], \mathcal{E}_{0}=E$, let $\partial: C \rightarrow E$ be the core-anchor, and additionally

$$
\nabla_{-1}=\psi^{c} \circ \sigma, \quad \nabla_{0}=\psi^{s} \circ \sigma,
$$

where $\psi^{s}, \psi^{c}$ are the side and the core representation respectively. Finally, we let $R$ be given by

$$
R(a, b)=\sigma[a, b]-[\sigma(a), \sigma(b)], \quad a, b \in \Gamma(A) .
$$

In the last part of this section we describe the 2-term representation up to homotopy corresponding to the VB-algebroid ( $\mathrm{D} E_{A} \Rightarrow \mathrm{D} E ; A \Rightarrow M$ ) from Proposition 3.13 . So let $A \Rightarrow M$ be a Lie algebroid, and let $E$ be a representation of it. We denote by $\nabla$ the flat $A$-connection in $E$. We have already seen that the core of ( $\left.\mathrm{D} E_{A} \Rightarrow \mathrm{D} E ; A \Rightarrow M\right)$ is (canonically isomorphic to) $A$. So there is a short exact sequence of vector bundles

$$
0 \rightarrow \operatorname{Hom}(\mathrm{D} E, A) \rightarrow \widehat{A} \rightarrow A \rightarrow 0
$$

where $\widehat{A}$ is the fat algebroid. We begin describing $\widehat{A}$. Denote by $p: A \rightarrow M$ the projection.

Lemma 4.5 The fiber $\widehat{A}_{x}$ of the fat algebroid $\widehat{A} \Rightarrow M$ of $\left(\mathrm{D} E_{A} \Rightarrow \mathrm{D} E ; A \Rightarrow M\right)$ over a point $x \in M$ consists of pairs $(a, h)$, where $a \in A_{x}$ and $h: \mathrm{D}_{x} E \rightarrow T_{a} A$ is a linear map such that $d p \circ h=\sigma$. The projection $\widehat{A} \rightarrow A$ maps $(a, h)$ to a.

Proof: The claim immediately follows from the isomorphism $\mathrm{D} E_{A} \cong T A_{d}{ }^{\ngtr}{ }_{\sigma} \mathrm{D} E$.

Remark 4.6 The map $\Gamma(A) \rightarrow \Gamma(\widehat{A}), a \mapsto \mathrm{D} a$ splits the short exact sequence

$$
0 \rightarrow \Gamma(\operatorname{Hom}(\mathrm{DE}, A)) \rightarrow \Gamma(\widehat{A}) \rightarrow \Gamma(A) \rightarrow 0
$$

in the category of vector spaces. In particular, a section of $\widehat{A} \rightarrow M$ can be seen as a pair $(a, \varphi)$ where $a \in \Gamma(A)$ and $\varphi \in \Gamma(\operatorname{Hom}(\mathrm{D} E, A))$. We now take this point of view to describe a little bit more explicitly (the core-anchor and) the core, the side representations and the Lie bracket in $\Gamma(\widehat{A})$. Actually, it easily follows from the definition of the Lie algebroid $\mathrm{D} E_{A} \Rightarrow \mathrm{D} E$ that

- the core-anchor $\partial: A \rightarrow D E$ agrees with the $A$-connection $\nabla$ in $E$;

- the side representation is given by $\psi_{\mathrm{D} a}^{s}=\left[\nabla_{a},-\right]$ and $\psi_{\varphi}^{s}=-\nabla \circ \varphi$,

- the core representation is given by $\psi_{\mathrm{D} a}^{c}=[a,-]$ and $\psi_{\varphi}^{c}=-\varphi \circ \nabla$;

- the Lie bracket in $\Gamma(\widehat{A})$ is given by

$$
[\mathrm{D} a, \mathrm{D} b]=\mathrm{D}[a, b], \quad[\mathrm{D} a, \varphi]=\mathcal{L}_{a} \varphi, \quad[\varphi, v]=v \circ \nabla \circ \varphi-\varphi \circ \nabla \circ v ;
$$

for all $a, b \in \Gamma(A)$, and $\varphi, v \in \Gamma(\operatorname{Hom}(\mathrm{D} E, A))$. Here $\mathcal{L}_{a} \varphi$ is the bundle map $\mathrm{D} E \rightarrow A$ given by

$$
\left(\mathcal{L}_{a} \varphi\right)(\Delta):=[a, \varphi(\Delta)]-\varphi\left(\left[\nabla_{a}, \Delta\right]\right), \quad \Delta \in \Gamma(\mathrm{D} E) .
$$


Now we choose a splitting of 4.19 . It follows from Lemma 4.5 that such a splitting is equivalent to a $\mathrm{D} E$-connection $\mathcal{D}$ in $A$. Namely, the connection $\mathcal{D}$ corresponds to the splitting $\sigma_{\mathcal{D}}: A \rightarrow \widehat{A}$ given by

$$
\sigma_{\mathcal{D}}(a):=\mathrm{D} a-\mathrm{d}_{\mathcal{D}} a, \quad a \in \Gamma(A),
$$

where $\mathrm{d}_{\mathcal{D}}: \Gamma(A) \rightarrow \Gamma(\operatorname{Hom}(\mathrm{D} E, A))$ is the connection differential. Hence, we get a 2-term representation up to homotopy $(\mathcal{E}, D)$ of $A$ by putting $\mathcal{E}=A[1] \oplus \mathrm{D} E$ and

$$
D=\partial+\nabla_{-1}+\nabla_{0}+R
$$

with

$$
\partial=\nabla, \quad \nabla_{-1}=\psi^{s} \circ \sigma_{\mathcal{D}}, \quad \nabla_{0}=\psi^{c} \circ \sigma_{\mathcal{D}},
$$

and

$$
R(a, b)=\sigma_{\mathcal{D}}[a, b]-\left[\sigma_{\mathcal{D}}(a), \sigma_{\mathcal{D}}(b)\right], \quad a, b \in \Gamma(A) .
$$

A straightforward computation using Remark 4.6 now shows that

$$
\left(\nabla_{-1}\right)_{a} \Delta=\left[\nabla_{a}, \Delta\right]+\nabla_{\mathcal{D}_{\Delta} a}, \quad\left(\nabla_{0}\right)_{a} b=[a, b]+\mathcal{D}_{\nabla_{b}} a
$$

and

$$
\begin{aligned}
R(a, b) \Delta= & {\left[\mathcal{D}_{\Delta} a, b\right]+\left[a, \mathcal{D}_{\Delta} b\right]-\mathcal{D}_{\Delta}[a, b] } \\
& -\mathcal{D}_{\left[\nabla_{a}, \Delta\right]} b+\mathcal{D}_{\left[\nabla_{b}, \Delta\right]} a+\mathcal{D}_{\nabla_{\mathcal{D}_{\Delta^{b}}}} a-\mathcal{D}_{\nabla_{\mathcal{D}_{\Delta^{a}}}} b,
\end{aligned}
$$

for all $a, b \in \Gamma(A)$, and all $\Delta \in \Gamma(\mathrm{D} E)$.

Summarizing, we proved the following

Proposition 4.7 Let $A \Rightarrow M$ be a Lie algebroid and let $E$ be a representation of $A$ with flat $A$ connection $\nabla$. Additionally, let $\mathcal{D}$ be a (not necessarily flat) $\mathrm{D} E$-connection in A. These data define a 2 -term representation up to homotopy $(\mathcal{E}, D)$ of $A$, where $\mathcal{E}=A[1] \oplus \mathrm{D} E$, and $D=\nabla+\nabla_{-1}+\nabla_{0}+R$, with $\nabla_{-1}, \nabla_{0}$, and $R$ being given by (4.27) and (4.28). The representation up to homotopy $(\mathcal{E}, D)$ does only depend on $(E, \nabla)$, in particular it is independent of $\mathcal{D}$, up to isomorphisms.

\section{Acknowledgements}

The authors thank Iakovos Androulidakis for useful discussions. They are also thankful to the anonymous referee for remarks that simplified a lot various proofs. AGT is supported by a FWO postdoctoral fellowship and further acknowledges the partial support received, during the preparation of this paper, by the GNSAGA (INdAM), the Centre for Mathematics of the University of Coimbra (UID/MAT/00324/2013), and the FWO research project G083118N (Belgium). LV is member of the GNSAGA of INdAM, Italy.

\section{References}

[1] Arias Abad, C., Crainic, M.: Representations up to homotopy of Lie algebroids. J. Reine Angew. Math. 663 (2012), 91-126.

[2] Arias Abad, C., Crainic, M.: Representations up to homotopy and Bott's spectral sequence for Lie groupoids. Adv. Math. 248 (2013), 416-452.

[3] Bailey, M., Gualtieri, M.: Integration of generalized complex structures. preprint arXiv:1611.03850 (2016).

[4] Bruce, A. J., Grabowska, K., Grabowski, J.: Remarks on contact and Jacobi geometry. SIGMA Symmetry Integrability Geom. Methods Appl. 13 (2017), 59-80.

[5] Bruce, A. J., Grabowski, J., Vitagliano, L.: Representations up to homotopy from weighted Lie algebroids. J. Lie Theory 28.3 (2018), 711-733. 
[6] Bursztyn, H., Cabrera, A.: Multiplicative forms at the infinitesimal level. Math. Ann. 353.3 (2012), $663-705$.

[7] Bursztyn, H., Cabrera, A., Del Hoyo, M.: Vector bundles over Lie groupoids and algebroids. Adv. Math. 290 (2016), 163-207.

[8] Bursztyn, H., Drummond, T.: Lie theory of multiplicative tensors. preprint arXiv:1705.08579 (2017).

[9] Cabrera, A., Drummond, T.: Van Est isomorphism for homogeneous cochains. Pacific J. Math. 287 (2017), 297-336.

[10] Costa, B. T., Forger, M., Pegas, L. H.: Lie Groupoids in Classical Field Theory I: Noether's Theorem. J. Geom. Phys. 131 (2018) 220-245.

[11] Coste, A., Dazord, P., Weinstein, A.: Groupoïdes symplectiques. Pub. Dép. Math. Lyon 2A (1987), 1-62.

[12] Crainic, M.: Differentiable and algebroid cohomology, van Est isomorphisms, and characteristic classes. Comment. Math. Helv. 78 (2003), 681-721.

[13] Crainic, M.: Generalized complex structures and Lie brackets. Bull. Braz. Math. Soc., New Series 42 (2011), $559-578$

[14] Crainic, M., Mestre, J. N., Struchiner, I.: Deformations of Lie groupoids. preprint arXiv:1510.02530 (2015). to appear in Int. Math. Res. Not. IMRN. https://doi.org/10.1093/imrn/rny221

[15] Crainic, M., Moerdisk, I.: Deformations of Lie brackets: cohomological aspects. J. Eur. Math. Soc. 10 (2008), 1037-1059.

[16] Crainic, M., Salazar, M. A., Struchiner, I.: Multiplicative forms and Spencer operators. Math. Z. 279 (2015), 939-979.

[17] Dazord, P.: Intégration d'algèbres de Lie locales et groupoïdes de contact. C. R. Acad. Sci Paris, Sér. I 320 (1995), 959-964.

[18] Del Hoyo, M, Ortiz, C.: Morita equivalences of vector bundles. preprint arXiv:1612.09289 (2016). to appear in Int. Math. Res. Not. IMRN. https://doi.org/10.1093/imrn/rny149

[19] Del Hoyo, M, Trentinaglia, G.: Simplicial vector bundles over Lie groupoids. work in progress.

[20] De Paris, A., Vinogradov, A. M.: Fat manifolds and linear connections. World Scientific, 2009.

[21] Drinfel'D, V. G.: Hamiltonian structures on Lie groups, Lie bialgebras and the geometric meaning of classical Yang-Baxter equations. Dokl. Akad. Nauk 268.2 (1983), 285-287.

[22] Drummond, T., Egea, L.: Differential forms with values in VB-groupoids and its Morita invariance. J. Geom. Phys. 135 (2019), 42-69.

[23] Drummond, T., Jotz Lean, M., Ortiz, C.: VB-algebroid morphisms and representations up to homotopy. Differential Geom. Appl. 40 (2015), 332-357.

[24] Esposito, C., Tortorella, A. G., Vitagliano, L.: Multiplicative multiderivations on VB-groupoids and algebroids (in preparation).

[25] Grabowska K., Grabowski, J., Urbański, P.: Lie Brackets on Affine Bundles. Ann. Glob. Anal. Geom. 24 (2003), 101-130.

[26] Grabowski, J., Rotkiewicz, M.: Higher vector bundles and multi-graded symplectic manifolds. J. Geom. Phys. 59 (2009), 1285-1305.

[27] Gracia-Saz, A., Mehta, R. A.: Lie algebroid structures on double vector bundles and representation theory of Lie algebroids. Adv. Math. 223.4 (2010), 1236-1275.

[28] Gracia-Saz, A., Mehta, R. A.: VB-groupoids and representation theory of Lie groupoids. J. Symplectic Geom. 15.3 (2017), 741-783.

[29] Iglesias-Ponte, D., Laurent-Gengoux, C., Xu, P.: Universal lifting theorem and quasi-Poisson groupoids. J. Eur. Math. Soc. (JEMS) 14.3 (2012), 681-731.

[30] Iglesias-Ponte, D., Marrero, J. C.: Generalized Lie bialgebroids and Jacobi structures. J. Geom. Phys. 40 (2001), 176-199.

[31] Iglesias-Ponte, D., Marrero, J. C.: Generalized Lie bialgebras and Jacobi structures on Lie groups. Israel J. Math. 133 (2003), 285-320.

[32] Iglesias-Ponte, D., Marrero, J. C.: Jacobi groupoids and generalized Lie bialgebroids. J. Geom. Phys. 48 (2003), 385-425.

[33] Jotz Lean, M.: The leaf space of a multiplicative foliation. J. Geom. Mech. 4.3 (2012), 313-332.

[34] Jotz Lean, M.: Dirac groupoids and Dirac bialgebroids. preprint arXiv:1403.2934 (2014). to appear in J. Symplectic Geom. 
[35] Jotz Lean, M., Ortiz., C.: Foliated groupoids and infinitesimal ideal systems. Indag. Math. 25 (2014), 10191053.

[36] Jotz Lean, M., StiÉnon, M., Xu, P.: Glanon groupoids. Math. Ann. 364 (2016), 485-518.

[37] Kerbrat, Y., Souici-Benhammadi, Z.: Variétés de Jacobi et groupoïdes de contact. C. R. Acad. Sci. Paris, Sér. I 317 (1993), 81-86.

[38] Kosmann-Schwarzbach, Y.: Multiplicativity, from Lie groups to generalized geometry. Banach Center Publ. 110 (2016), 131-166.

[39] La Pastina P. P., Vitagliano, L.: Deformations of Linear Lie Brackets. preprint arXiv:1805.02108 (2018).

[40] La Pastina P. P., Vitagliano, L.: Deformations of vector bundles over Lie groupoids. (in preparation).

[41] Lê, H. V., Oн, Y.-G., Tortorella, A. G., Vitagliano, L.: Deformations of coisotropic submanifolds in Jacobi manifolds. J. Symplectic Geom. 16 (2018), 1051-1116.

[42] Lê, H. V., Tortorella, A. G., Vitagliano, L.: Jacobi bundles and the BFV-complex. J. Geom. Phys. 121.4 (2017), 347-377.

[43] Libermann, P.: On symplectic and contact groupoids. In: Differential Geometry and its applications, $29-45$. Silesian University, Opava, 1993.

[44] Mackenzie, K. C. H.: Double Lie algebroids and second-order geometry. I. Adv. Math. 94.2 (1992), 180-239.

[45] Mackenzie, K. C. H.: Double Lie algebroids and second-order geometry. II. Adv. Math. 154.1 (2000), 46-75.

[46] Mackenzie, K. C. H.: General theory of Lie groupoids and Lie algebroids, vol. 213. in London Math. Soc. Lecture Note Ser. Cambridge University Press, 2005.

[47] Mackenzie, K. C. H., Xu, P.: Classical lifting processes and multiplicative vector fields. Quarterly J. Math. 49.1 (1998) 59-85.

[48] Marle, C.-M.: On Jacobi manifolds and Jacobi bundles. In: Dazord, P., Weinstein, A. (Eds.): Symplectic Geometry, Groupoids, and Integrable Systems, vol. 20 in Math. Sci. Res. Inst. Publ., 227-246. Springer, 1991.

[49] Menta, R. A.: Q-algebroids and their cohomology. J. Symplectic Geom. 7 (2009), 263-293.

[50] Menta, R. A.: Q-groupoids and their cohomology. Pacific J. Math. 242 (2009), 311-332.

[51] Menta, R. A.: Lie algebroid modules and representations up to homotopy. Indag. Math. 25 (2014), $1122-1134$.

[52] Ortiz, C.: Multiplicative Dirac structures on Lie groups. C. R. Acad. Sci. Paris, Sér. I 346 (2008), 1279-1282.

[53] Ortiz, C.: Multiplicative Dirac structures. Pacific J. Math. 266 (2013), 329-365.

[54] Salazar, M. A.: Pfaffian groupoids. PhD thesis, University of Utrecht, 2013.

[55] Stefanini, L.: On the integration of $\mathcal{L} \mathcal{A}$-groupoids and duality for Poisson groupoids. Trav. Math. 17 (2007), $39-59$.

[56] Stiénon, M., Xu, P.: Poisson quasi-Nijenhuis manifolds. Comm. Math. Phys. 270 (2007), 709-725.

[57] Vă̌ntrob, A. Yu.: Lie algebroids and homological vector fields. Uspekhi Mat. Nauk 52 (1997), $161-162$.

[58] Vitagliano, L.: Dirac-Jacobi Bundles. J. Symplectic Geom. 16.2 (2018), 485-561.

[59] Vitagliano, L.: Representations of homotopy Lie-Rinehart algebras. Math. Proc. Camb. Phil. Soc. 158 (2015), $155-191$.

[60] Vitagliano, L., Wade, A.: Generalized contact bundles. C. R. Math. 354 (2016), 313-317.

[61] Vitagliano, L., Wade, A.: Holomorphic Jacobi manifolds and complex contact groupoids. preprint arXiv:1710.03300 (2017).

[62] Voronov, T.: Q-manifolds and Mackenzie theory. Comm. Math. Phys. 315 (2012), 279-310.

[63] Weinstein, A.: Symplectic groupoids and Poisson manifolds. Bull. Amer. Math. Soc. 16 (1987), 523-557.

[64] Weinstein, A., Xu, P.: Extensions of symplectic groupoids and quantization. J. Reine Angew. Math. 417 (1991), 159-189.

[65] Yano, K., Ishinara, S.: Tangent and cotangent bundles. Marcel Dekker, Inc., New York, 1973.

[66] Yudilevich, O.: Lie Pseudogroups à la Cartan from a modern perspective. PhD thesis, University of Utrecht, 2016.

[67] Zakrzewski, S.: Quantum and classical pseudogroups. I. Union pseudogroups and their quantization. Comm. Math. Phys. 134 (1990), 347-370.

[68] Zakrzewski, S.: Quantum and classical pseudogroups. II. Differential and symplectic pseudogroups. Comm. Math. Phys. 134 (1990), 371-395. 